Der Medizinischen Fakultät der Georg-August-Universität Göttingen eingereicht von Prof. Dr. med. Brit Mollenhauer

\title{
Evaluation eines Riechtests im Rahmen der De-Novo-Parkinson-Kassel-Studie
}

\author{
INAUGURAL-DISSERTATION \\ zur Erlangung des Doktorgrades \\ der Medizinischen Fakultät der \\ Georg-August-Universität zu Göttingen
}

\author{
vorgelegt von \\ Sonja Erika Anna Hippel \\ aus \\ Berlin
}

Göttingen 2018 
Dekan:

Referent/in:

Ko-Referent/in:

Drittprüfer/in:

Datum der mündlichen Prüfung:
Prof. Dr. rer. nat. H. K. Kroemer

Prof. Dr. med. Brit Mollenhauer

PD Dr. Marcus Niebert

Prof. Dr. Margarete Schön

21.11.2018 
Hiermit erkläre ich, die Dissertation mit dem Titel „Evaluation eines Riechtests im Rahmen der De-Novo-Parkinson-Kassel-Studie" eigenständig angefertigt und keine als die von mir angegebenen Quellen und Hilfsmittel verwendet zu haben.

Berlin, den 21.02.2018 


\section{INHALTSVERZEICHNIS}

INHALTSVERZEICHNIS

ABBILDUNGSVERZEICHNIS III

TABELLENVERZEICHNIS IV

ABKÜRZUNGSVERZEICHNIS .. V

1 EINLEITUNG 1

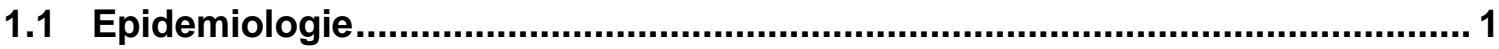

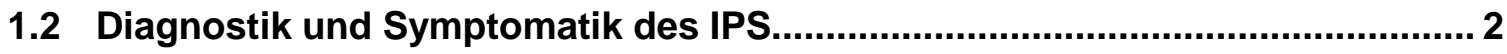

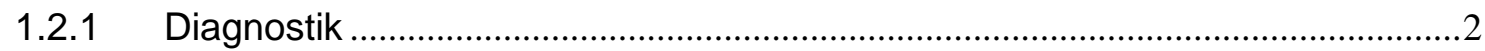

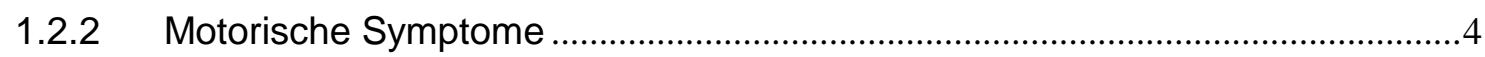

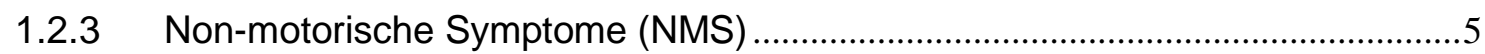

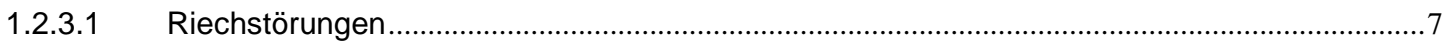

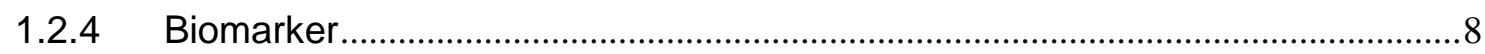

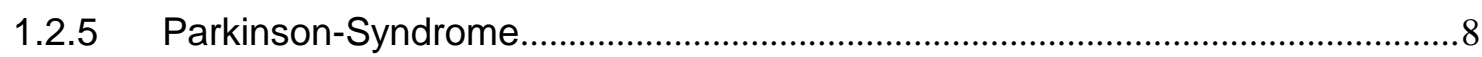

1.3 Pathophysiologische Prozesse des IPS ...................................................... 10

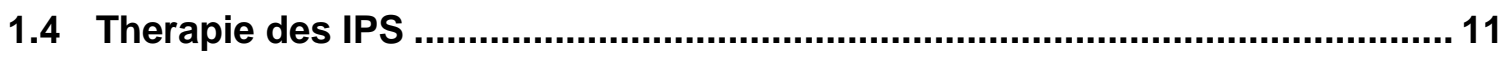

2 MATERIAL UND METHODEN

2.1 Die DeNoPa-Kassel-Studie ........................................................................ 13

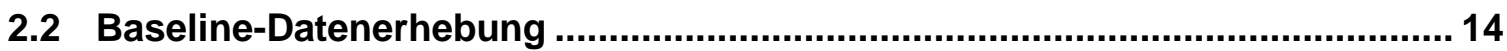

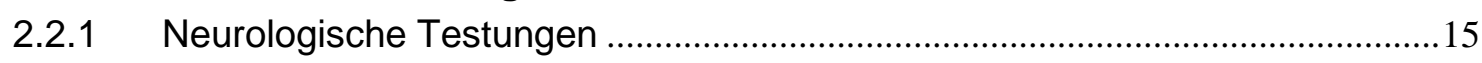

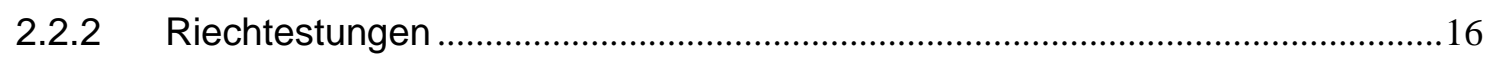

2.2.2.1 Subjektive Riechtestungen und Fragebögen zu non-motorischen Symptomen (persönliche Einschätzung, PD NMS-Fragebogen Frage 2, Skala zu non-motorischen Symptomen Frage 28) ...........16

2.2.2.2 Objektive Riechtestungen (Schwellentest, Diskriminationstest, Identifikationstest) ................17

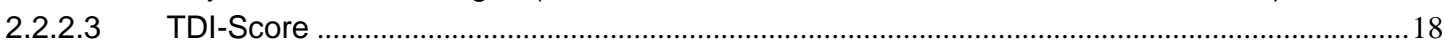

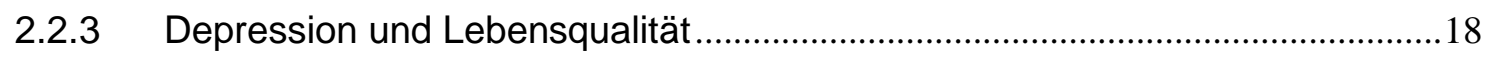

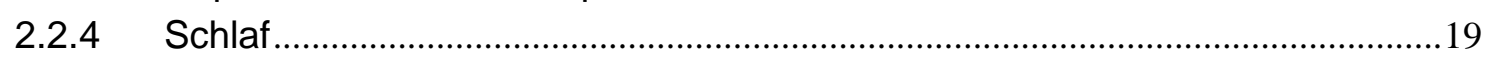

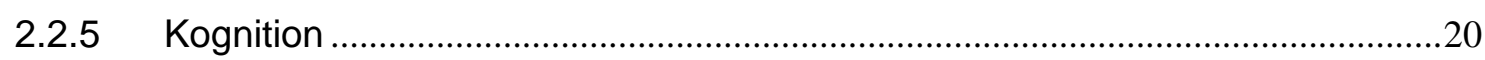

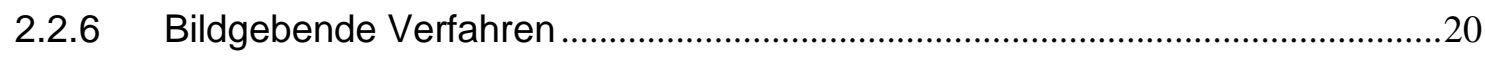

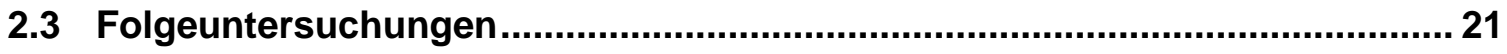

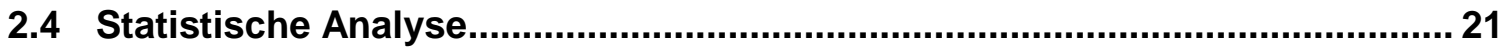

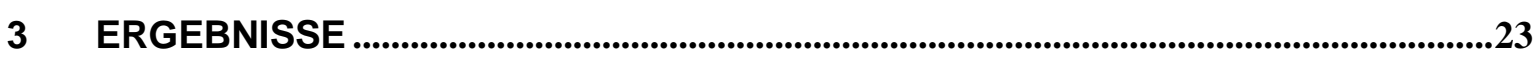

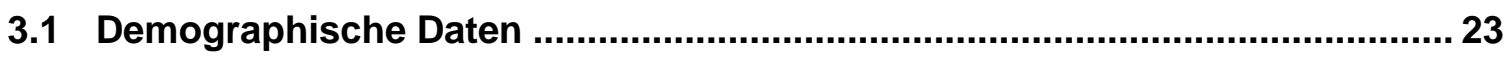

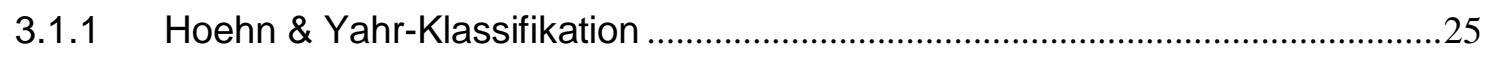

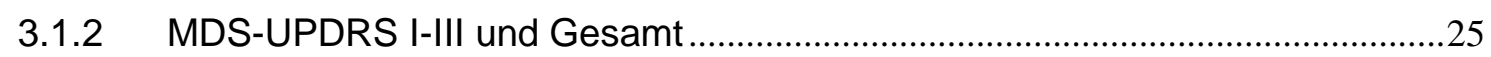

3.1.3 Dauer der Erkrankung nach Erstmanifestation ..................................................26 


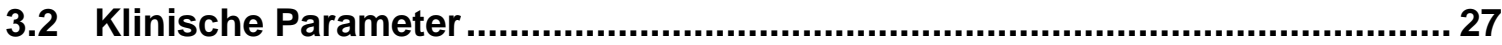

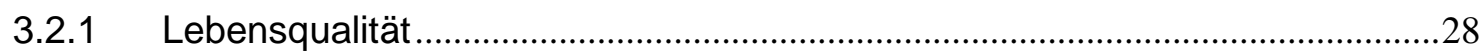

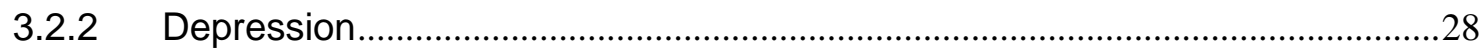

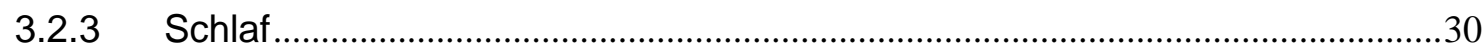

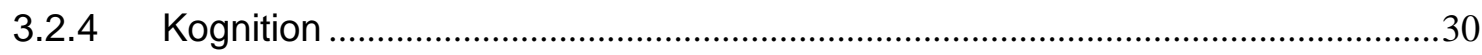

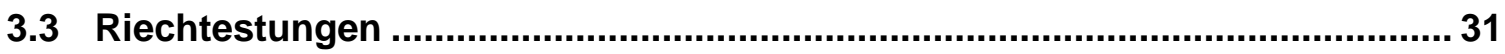

3.3.1 Subjektive Bewertungen des Riechvermögens ..............................................

3.3.1.1 Persönliche Einschätzung vor der Durchführung der Riechtestungen mithilfe der Sniffin' Sticks 32

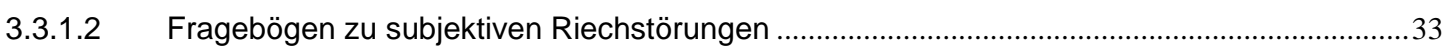

3.3.2 Objektive Riechtestungen zur Schwelle, Diskrimination und Identifikation

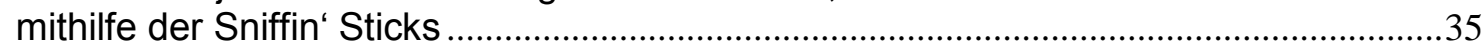

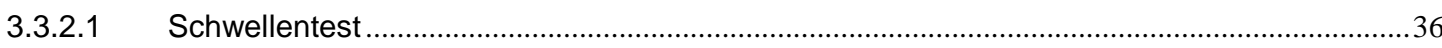

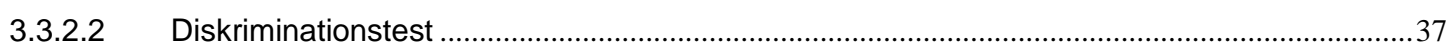

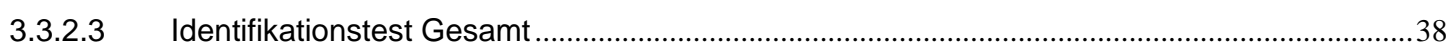

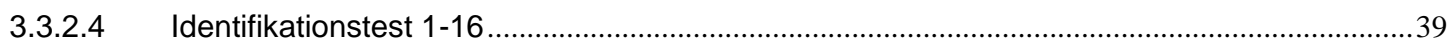

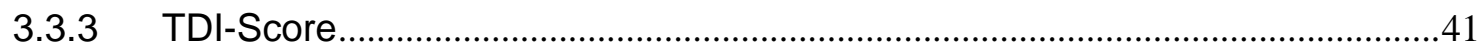

3.3.4 Sensitivität, Spezifität und Youden-Index des Riechidentifikationstests...........42

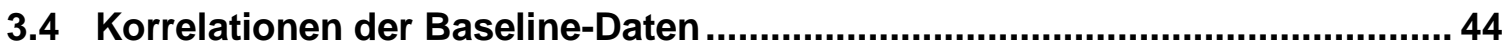

3.4.1 Korrelationen Riechtest Schwelle, Diskrimination und Identifikation Gesamt.44

3.5 Folgeuntersuchung der Riechtestungen nach 24 Monaten ........................... 49

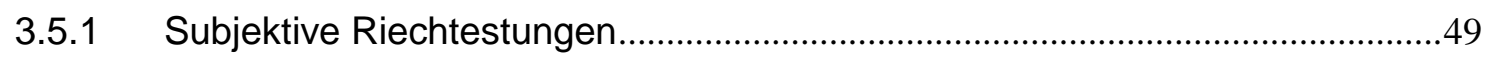

3.5.2 Objektive Riechtestungen zur Identifikation von 16 Qualitäten mithilfe der

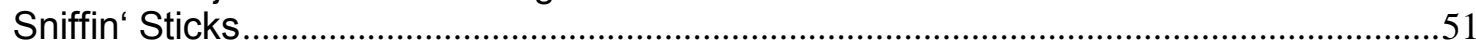

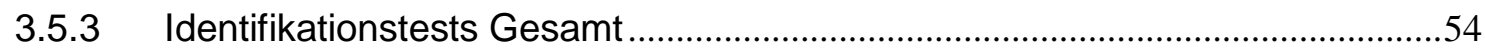

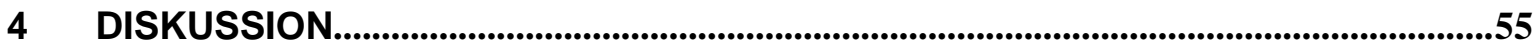

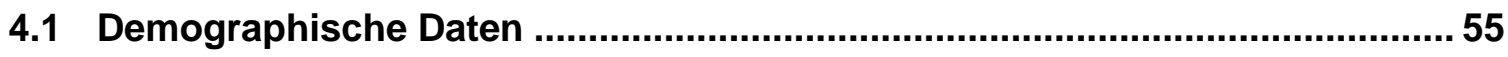

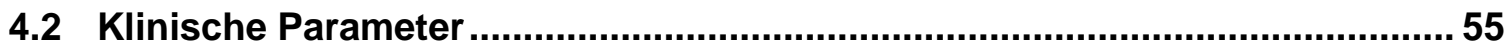

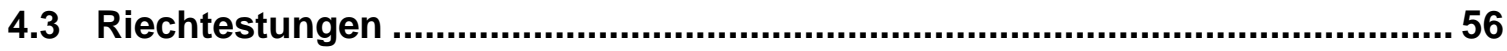

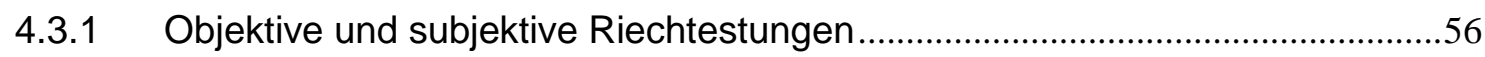

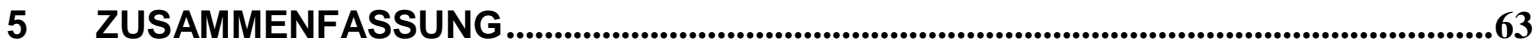

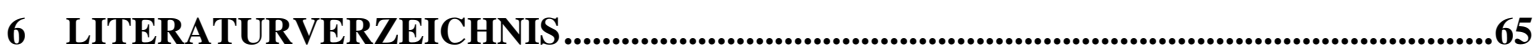

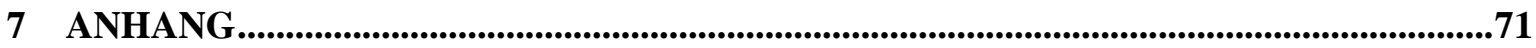




\section{ABBILDUNGSVERZEICHNIS}

Abbildung 1 Dauer der Erkrankung der Parkinsonpatienten ................................................................. 26

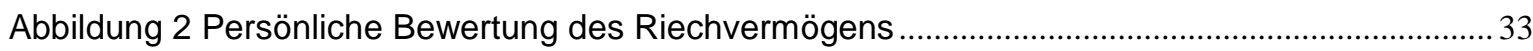

Abbildung 3 Frage 28 der Skala zu non-motorischen Symptomen, Häufigkeit der Beschwerden ... 34

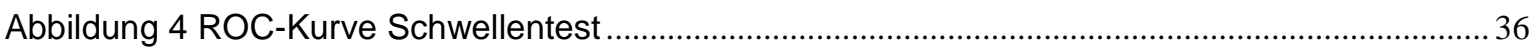

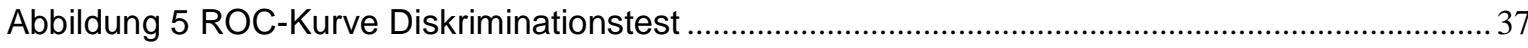

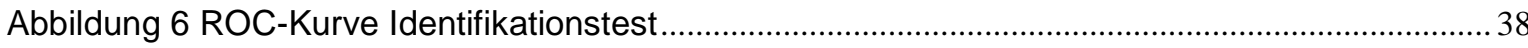

Abbildung 7 Korrekte Antworten im Sniffin‘ Sticks-Identifikationstest 1-8 …......................................... 39

Abbildung 8 Korrekte Antworten im Sniffin' Sticks-Identifikationstest 9-16 _......................................... 39

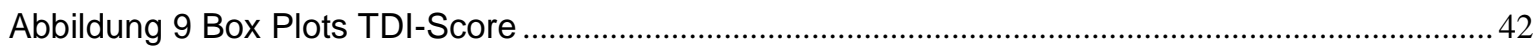

Abbildung 10 Identifikationstestung 1-16, Vergleich Baseline- und Folgeuntersuchung .....................51

Abbildung 11 Gesamtscore der Identifikationstestungen, Baseline und Folgeuntersuchung ............54 


\section{TABELLENVERZEICHNIS}

Tabelle 1 Diagnosekriterien der Parkinson-Erkrankung (Poewe und Deuschl 2012)........................... 3

Tabelle 2 Non-motorische Symptome im Rahmen einer Parkinsonerkrankung (Reichmann 2010) ..5

Tabelle 3 Parkinson-Syndrome (DGN 2016) 9

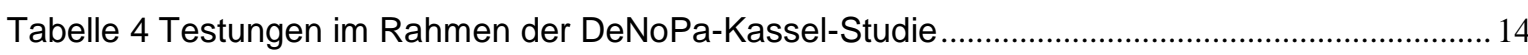

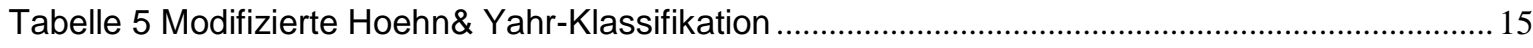

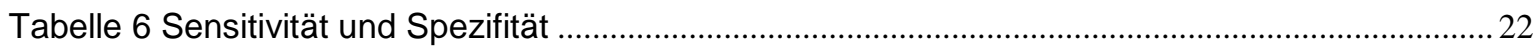

Tabelle 7 Demographische Daten, MDS UPDRS und Hoehn \& Yahr-Klassifikation der Parkinsonpatienten (PD) und der gesunden Kontrollgruppe (HC) für die Baseline-Testung und die Folgetestung nach 24 Monaten und der darauf bezogene $\mathrm{p}$-Wert.

Tabelle 8 Klinische Parameter: Testungen zu Depression (BDI, GDS, MADRS), der Lebensqualität (PDQ-39), REM-Schlafstörungen (Polysomnographie) und Kognition (MMST, Uhrentest, MoCA) der Parkinsonpatienten $(\mathrm{PD})$ und der Kontrollgruppe $(\mathrm{HC})$, vergleichend dargestellt für die Baseline- und Folgetestung nach 24 Monaten

Tabelle 9 Subjektive Bewertung des Riechvermögens: Persönliche Einschätzung, Befragung durch PD NMS-Fragebogen Frage 2, Skala zu non-motorischen Symptomen Frage 28 und deren

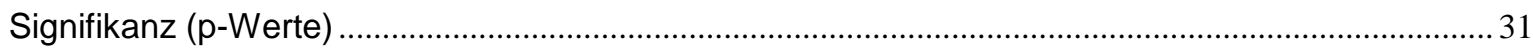

Tabelle 10 Objektive Riechtestungen (Schwellentest, Diskriminationstest, Identifikationstest) im Rahmen der Baseline-Datenerfassung, Durchführung mithilfe der Sniffin‘ Sticks, Signifikanz ( $p$-Wert)

Tabelle 11 Patienten Identifikationstests 1-16, Häufigkeitstabelle und p-Werte, Anzahl (\%) 40

Tabelle 12 Sensitivität, Spezifität und Youden-Index (= Sensitivität+ Spezifität- 1$)$ des Identifikationstests 1-16 der Patienten

Tabelle 13 Subjektive Riechtestungen, Vergleich Baseline- und Folgeuntersuchung nach 24 Monaten, $p$-Wert für die Patienten in der Baseline (BL)- und Folgeuntersuchung (FU)

Tabelle 14 Korrekte Antworten im Identifikationstest 1-16 und Gesamt, Baseline- und Folgeuntersuchung, Signifikanz ( $p$-Wert) BL vs. FU. 


\section{ABKÜRZUNGSVERZEICHNIS}

AUC

BDI

DAT Scan

DeNoPa

GDS

HC

$H \& Y$

IPS

MADRS

MDS UPDRS I-III

MMST

MoCA

MSA

PD

PD NMS Fragebogen 2

PET-CT

REM

ROC

Skala PD NMSS 28

SPECT

PDQ- 39

TDI-Score

UPDRS I-III

UPSIT
Area Under The Curve

Beck-Depressions-Inventar

Dopamintransporter-Szintigraphie

De-Novo-Parkinson

Geriatrische Depressionsskala

Healthy Control- gesunde Kontrollgruppe

Hoehn \& Yahr-Klassifikation

Idiopathisches Parkinsonsyndrom

Montgomery Asberg Depression Rating Scale

Movement Disorder Society-Unified Parkinson's Disease Rating Scale I-III

Mini-Mental-Status-Test

Montreal Cognitive Assessment

Multiple Systematrophie

Parkinson's Disease, Parkinsonerkrankung

Parkinson's Disease Non-Motor Symptoms

Questionnaire, Frage 2

Positronen-Emissions-Computertomographie

Rapid Eye Movement

Receiver Operator Characteristic

Skala zur Erfassung non-motorischer Symptome bei der

Parkinson-Erkrankung, Frage 28

Single Photon Emission-Computed Tomography

Parkinson's Disease Questionnaire-39

Threshold, Discrimination, Identification-Score, Summe aus

Schwellen-, Diskriminations- und Identifikationstest

Unified Parkinson's Disease Rating Scale I-III

University of Pennsylvania Smell Identification Test 


\section{EINLEITUNG}

Morbus Parkinson beschäftigt die medizinische Welt schon seit fast 200 Jahren, seitdem James Parkinson 1817 seine ersten Beobachtungen zu der Erkrankung in "An Essay on the Shaking Palsy" verfasste und der Erkrankung somit ihren Namen gab. Seinen Beobachtungen zufolge beschreibt er die sogenannte Schüttellähmung als unfreiwilliges Zittern mit daraus resultierender Abnahme der Muskelkraft und einer Neigung des Rumpfes nach ventral. Auffällig dabei ist ein veränderter Gang, der Intellekt und die Sinne bleiben unbeeinträchtigt (Parkinson 2002).

Nach wie vor ist die Parkinson-Erkrankung eine Erkrankung, deren Progression bisher nicht aufgehalten werden kann (Chen et al. 2013). Um aber langfristig eine neuroprotektive Therapie entwickeln zu können, die den Verlauf der Erkrankung unterbrechen oder beeinflussen kann, macht man sich Beobachtungen non-motorischer Symptome der Erkrankung zu Nutze. Ziel ist es, durch die non-motorischen Symptome diagnostische Biomarker einzuführen, die eine frühere Diagnose ermöglichen. In der vorliegenden Arbeit wurden die im Rahmen der DeNoPa-Kassel-Studie umfangreich erhobenen Daten an De-Novo-Parkinsonpatienten ausgewertet. Im Vordergrund der Untersuchungen dieser Dissertation stehen die mithilfe der Sniffin Sticks durchgeführten Riechtestungen. Diese sollen die olfaktorischen Defizite bei Parkinson-Patienten darstellen und die Entwicklung im Verlauf der Erkrankung durch eine Auswertung von Messergebnissen an zwei Zeitpunkten herausarbeiten. Dies soll einen wissenschaftlichen Beitrag zu den Kenntnissen über den Beginn und den Verlauf des idiopathischen Parkinson-Syndroms leisten und zu der Entwicklung von diagnostischen Biomarkern beitragen.

\subsection{Epidemiologie}

Das idiopathische Parkinsonsyndrom ist die zweithäufigste neurodegenerative Erkrankung nach Morbus Alzheimer und tritt vermehrt im fortgeschrittenen Alter auf. Sie wird nachweislich häufiger bei Männern als bei Frauen diagnostiziert (Wirdefeldt et al. 2011). Die Prävalenz der Erkrankung liegt in Deutschland bei 1.800/ 100.000 Einwohner bei den > 65-Jährigen (DGN 2008). Die Inzidenz des idiopathischen Parkinsonsyndroms nimmt mit steigendem Alter zu (Mayeux et al. 1995; van Den Ee- 
den 2003). Selten tritt das sogenannte frühe Parkinsonsyndrom (Young-onset Parkinson's Disease) auf. Dies beschreibt einen Krankheitsbeginn zwischen dem 21. und dem 40. Lebensjahr. Zeigen sich bereits vor dem 20. Lebensjahr Symptome der Erkrankung, wird dies als juveniles Parkinsonsyndrom bezeichnet (Quinn et al. 1987). Insgesamt sind 0,15 \% der Bevölkerung vom idiopathischen Parkinsonsyndrom betroffen, bei den > 50-Jährigen sind es sogar $0,5 \%$ (Stoof et al. 1999). Schätzungen zufolge wird sich die Anzahl der Erkrankten im Jahre 2030 auf 8,7-9,3 Millionen belaufen, was einer Verdoppelung der Patienten im Vergleich zum Jahre 2005 entspräche. Dieser Annahme liegt die ansteigende Lebenserwartung der Bevölkerung weltweit zu Grunde (Dorsey et al. 2007).

\subsection{Diagnostik und Symptomatik des IPS}

Die Erkrankung umfasst eine große Bandbreite non-motorischer und motorischer Symptome, die die Lebensqualität der Patienten entscheidend beeinflussen (Schrag et al. 2000).

\subsubsection{Diagnostik}

Für die Diagnosestellung des idiopathischen Parkinsonsyndroms werden, auf der klinischen Untersuchung basierend, die Kriterien der United Kingdom Parkinson's Disease Society Brain Bank zu Grunde gelegt. Diese setzen eine Bradykinese in Verbindung mit mindestens einem weiteren Symptom wie Rigor, Tremor oder posturaler Instabilität voraus, die nicht visuell, zerebellär, vestibulär oder durch eine propriozeptive Dysfunktion bedingt ist (Hughes et al. 1992).

Um eine klinische Diagnose zu stellen, kann des Weiteren der L-Dopa- und der Apomorphin-Test verwendet werden. Üblicherweise werden dem Patienten dabei 200 mg Levodopa bzw. 50 g/ kg Körpergewicht Apomorphin verabreicht, um anschließend erneut den Unified Parkinson's Disease Rating Scale (UPDRS)-Test durchzuführen. Tritt eine Verbesserung des Ergebnisses von $\geq 30 \%$ auf, geht man von einer Parkinsonerkrankung aus (Reichmann 2010). Diese Testung und die überarbeitete Version, der Movement Disorder Society-Unified Parkinson's Disease Rating Scale (MDS-UPDRS), werden in Kapitel 2.2.1 erläutert.

Die weiteren diagnostischen Kriterien werden in Tabelle 1 dargestellt. 


\section{Diagnose eines Parkinson-Syndroms durch Feststellung von Akinese/ Bradykinese}

Verlangsamung bei der Initiierung und Durchführung willkürlicher Bewegungen, progressive Verlangsamung und Abnahme der Amplitude bei repetitiven Bewegungen in Verbindung mit mindestens einem folgenden Symptom:

- Muskulärer Rigor

- Ruhetremor (Abnahme bei Bewegung)

- Posturale Instabilität

\section{Vorhandensein unterstützender Kriterien}

- Einseitiger Beginn und persistierende Asymmetrie im Krankheitsverlauf

- Klassischer Ruhetremor

- Eindeutig positives Ansprechen auf L-Dopa (> 30\% UPDRS motorisch)

- Anhaltende L-Dopa-Ansprechbarkeit über mehr als 5 Jahre

- Auftreten von L-Dopa-induzierten choreatischen Dyskinesien

- Langsame klinische Progression mit Krankheitsverlauf über mehr als 10 Jahre

\section{Fehlen von Ausschlusskriterien}

\subsection{Hinweis für ein symptomatisches Parkinsonsyndrom}

- Behandlung mit Neuroleptika oder Exposition gegenüber anderen Parkinson-Krankheitsauslösenden Medikamenten oder Toxinen in zeitlichem Zusammenhang mit Erstmanifestation der Parkinson-Syndrome

- Nachweis struktureller Basalganglienveränderungen, frontaler Tumoren oder eines Hydrocephalus communicans in der zerebralen Bildgebung

- Wiederholte ischämische Insulte, die mit einer stufenweisen Verschlechterung der Parkinson-Symptomatik assoziiert waren

- Rezidivierende Schädelhirntraumata in der Vorgeschichte

- Diagnostisch gesicherte Enzephalitis in der Vorgeschichte

- Remission über längere Perioden

\subsection{Warnsymptome, die auf ein atypisches Parkinson-Syndrom hinweisen können}

- Nichtansprechen auf hohe Dosen L-Dopa (1000 mg/ Tag) nach Ausschluss einer Malresorption (z. B. im Dünndarmbereich) über mehrere Monate 
- Im Krankheitsverlauf frühzeitig auftretende schwere Störungen des autonomen Nervensystems (orthostatische Hypotension, Synkopen, Impotenz oder verringerte genitale Empfindlichkeit, Urininkontinenz oder -retention, Anhidrose)

- Zerebelläre Zeichen

- Positives Babinski-Zeichen, soweit keine andere Ursache bekannt (z. B. Apoplex)

- Ausgeprägte Antecollis

- Supranukleäre vertikale Blickparese/ frühe posturale Instabilität und Stürze

- Apraxie

- Innerhalb des ersten Jahres auftretende Demenz

- Innerhalb des ersten Jahres auftretende fluktuierende visuelle Halluzinationen

Klinisch gibt es zurzeit keine diagnostischen Verfahren, die eine endgültige Diagnose des idiopathischen Parkinsonsyndroms zulassen. Ausschließlich die pathologische Untersuchung post mortem kann den Nachweis einer Degeneration der dopaminergen Neurone und das Vorhandensein von Lewy Körperchen und Neuriten nachweisen und damit eine sichere Diagnose stellen (Kalia und Lang 2015).

\subsubsection{Motorische Symptome}

Die einseitige Bradykinese, Rigor und Tremor werden auch heute noch als die Kardinalsymptome des idiopathischen M. Parkinson gesehen (Alves et al. 2008). Dabei postulieren Hoehn und Yahr (2001), dass 70\% der Parkinsonpatienten einen Tremor aufweisen und 90\% eine Akinese oder Rigor.

Die Bradykinese wird unter anderem klinisch sichtbar durch die Hypomimie, einseitig reduzierte Schwingbewegungen des Armes und ein verändertes Schriftbild (Reichmann 2010). Bei passiver Bewegung einer Extremität des Patienten tritt ein erhöhter Widerstand, der sogenannte Rigor, auf (Jankovic 2008). Der bei der Parkinsonerkrankung auftretende einseitige, distal betonte Ruhetremor zeigt eine Frequenz von 4 bis $6 \mathrm{~Hz}$. Er entspricht an der Hand einem Supinations-PronationsTremor, kann jedoch auch das Kinn, die Lippen, den Kiefer und die Beine betreffen (Jankovic 2008). Ein weiteres Symptom, das das Gangbild bei dem idiopathischen Parkinson-Syndrom beeinflussen kann, ist das sogenannte freezing of gait. Darunter versteht man eine episodisch auftretende Gangstörung, bei der der Patient eine 
Bewegung nicht starten oder fortführen kann (Snijders et al. 2008), auch als Akinesie bezeichnet. Häufig ist dieses Symptom die Ursache von Stürzen und Verletzungen (Bloem et al. 2004).

Eine posturale Instabilität entsteht durch den Verlust der posturalen Reflexe und zeichnet sich eher im mittleren Stadium der Erkrankung ab (Leitlinie idiopathisches Parkinsonsyndrom 2016).

\subsubsection{Non-motorische Symptome (NMS)}

Während die motorischen Symptome der Diagnosestellung des Morbus Parkinson zugrunde liegen, treten häufig weitere Symptome auf, die als non-motorische Symptome bezeichnet werden (Fahn 2003). Es wird angenommen, dass non-motorische Symptome, wie beispielsweise Anosmie, Schlafstörungen und autonome Störungen die Erstmanifestation vom idiopathischen Parkinsonsyndrom darstellen können und motorische Symptome häufig erst später auftreten (Tolosa und Pont-Sunyer 2011). Laut Gaenslen et al. (2011) machen sich diese Symptome bereits 10-15 Jahre, bevor motorische Defizite nachweisbar sind, bemerkbar. Diese sogenannte prodromale Phase ist wahrscheinlich ein Teil der Erkrankung und wird vor Auftreten der motorischen Symptome präsent (Gonera et al. 1997).

Tabelle 2 Non-motorische Symptome im Rahmen einer Parkinsonerkrankung (Reichmann 2010)

\section{Neuropsychiatrische Symptome}

- Anhedonie

- Apathie

- Depression

- Psychosen

- Demenz

- Angst

- Panikattacken

\section{Sensorik}

- Schmerz

- Olfaktorische Störungen 
- Gestörte Diskrimination von Farben

\section{Autonomes Nervensystem}

- Hypotension

- Blasenschwäche/ Inkontinenz

- Obstipation

- Impotenz

- Sialorrhoe

- Anhidrose

\section{Schlaf}

- Restless legs

- Insomnie

- Rapid Eye Movement (REM)-Schlaf-Verhaltensstörungen

- Exzessive Tagesmüdigkeit

- Fragmentation des Schlafs

- Schlafattacken

\section{Sonstiges}

- Seborrhö

- Verzerrtes Sehen

Obwohl non-motorische Symptome, wie REM-Schlaf-Verhaltensstörungen, die Lebensqualität der Patienten stark vermindern, sind die therapeutischen Möglichkeiten begrenzt (Muntean et al. 2014). Die häufig eingesetzten Präparate mit dem Wirkstoff L-Dopa scheinen, im Gegensatz zu den motorischen Symptomen, bei den non-motorischen Symptomen keine einschneidende Wirkung zu zeigen (Erro et al. 2013).

Bezogen auf die Morbidität und Mortalität stehen vor allem non-motorische Symptome wie Dysphagie, autonome Dysfunktion und Demenz, aber auch der gestörte Gleichgewichtssinn, im Vordergrund. Diese Symptome zeigen kein Ansprechen auf L-Dopa (Hely et al. 2005). 


\subsubsection{Riechstörungen}

Bereits 1975 schrieben Ansari und Johnson (1975), dass das idiopathische Parkinsonsyndrom mit einer Hyposmie einhergeht. Mittlerweile geht man davon aus, dass 97,8\% der Parkinsonpatienten eine Riechstörung aufweisen (Haugen et al. 2016). $\mathrm{Ob}$ die neuropathologischen Prozesse in der Riechbahn beginnen, ist nicht gesichert (Hawkes 1999). Nachgewiesen ist jedoch, dass in frühen Stadien der Erkrankung bereits olfaktorische Einschränkungen bestehen. Dies lässt wiederum die Vermutung aufkommen, dass die Hyposmie vor den motorischen Symptomen der Parkinsonerkrankung auftreten könnte (Tissingh et al. 2001). Laut Haehner et al. (2007) kann dies Jahre zuvor präsent werden. Nach Ross et al. (2008) besteht bei vorliegender Hyposmie ein erhöhtes Risiko innerhalb der nächsten 4 Jahre an Parkinson zu erkranken. Laut Ponsen et al. (2010) haben Verwandte ersten Grades mit einer idiopathischen Hyposmie ein Risiko von 12,5\% in den nächsten 5 Jahren am idiopathischen Parkinsonsyndrom zu erkranken. Demzufolge ist ein Riechtest im prämotorischen Stadium der Erkrankung eine sensitive Screening-Methode, auf die bei positivem Ergebnis weitere, für das idiopathische Parkinson-Syndrom spezifische Testungen folgen sollten (Berardelli et al. 2013).

Bezüglich der verschiedenen Parkinson-Syndrome gibt es laut Wenning et al. (1995) Unterschiede in der Schwere der Riechstörung. Während atypische Parkinsonsyndrome wie die Multisystematrophie (MSA), die progressive supranukleären Blickparese (PSP) und die kortikobasale Degeneration (CBD) eher mit einem normalen Riechvermögen oder milden Einschränkungen einhergehen, treten bei dem idiopathischen Parkinson-Syndrom (IPD) häufiger ausgeprägte Defizite auf. Die Parkinson-Syndrome werden in Kapitel 1.2.5. erläutert.

Bezüglich des Zusammenhangs zwischen der Erkrankungsdauer und der Ausprägung des Riechvermögens scheint die Studienlage nicht einheitlich. Während durch zahlreiche Studien nachgewiesen wurde, dass kein Zusammenhang zwischen der Erkrankungsdauer und der Ausprägung der Riechstörung besteht (Herting et al. 2008; Doty et al. 1989; Daum et al. 2000), beschreiben Tissingh et al. (2001) eine Signifikanz zwischen den Diskriminationstestungen und dem Krankheitsstadium. 


\subsubsection{Biomarker}

Biologische Marker stellen objektiv messbare Kriterien dar, die in der Medizin vielseitige Anwendung finden. Sie können beispielsweise als Hilfsmittel dienen, um Krankheiten zu diagnostizieren, die Schwere einer Erkrankung einzustufen, die Prognose einer Erkrankung zu formulieren oder um die klinischen Auswirkungen einer Behandlung zu verfolgen (Biomarkers Definitions Working Group 2001).

Der einzige klinische Biomarker, der momentan für das idiopathische Parkinsonsyndrom anwendbar ist, ist das Ansprechen auf eine dopaminerge Therapie (Mollenhauer und Zhang 2012). Des Weiteren existieren bildgebende Verfahren, wie das PET, DAT Scan oder SPECT, die den neuronalen Verlust des Dopamins darstellen können. Vermutlich werden die Ergebnisse dieser bildgebenden Verfahren durch eine dopaminerge Therapie beeinflusst. Um Patienten identifizieren zu können, bei denen das Risiko für eine Parkinsonerkrankung besteht, die sich in einem frühen Stadium der Erkrankung befinden, ist es wahrscheinlich, dass mehrere Messungen durchgeführt werden müssen. Zum Beispiel eine Kombination aus einer klinischen Testung, wie einem Riechtest, einem biochemischen Marker und einem bildgebenden Verfahren (Schapira 2013).

\subsubsection{Parkinson-Syndrome}

Die Parkinson-Syndrome werden in 4 Gruppen unterteilt, idiopathische, atypische, sekundäre und genetisch bedingte Parkinsonsyndrome. $75 \%$ der Syndrome entsprechen dem idiopathischen Parkinson-Syndrom. Allen Syndromen liegt das Vorhandensein einer Akinese in Kombination mit einem weiteren Symptom, Rigor, Ruhetremor oder posturaler Instabilität, zugrunde (DGN 2008). Die nachfolgende Tabelle zeigt, abgesehen von dem genetischen Parkinsonsyndrom, alle Formen des Parkinsonsyndroms. Genetische Parkinsonsyndrome können beispielsweise durch Mutationen im Parkin-, PINK1-, DJ1- und LRRK2- Gen entstehen und können sich dabei dem idiopathischen Parkinsonsyndrom ähnlich darstellen (Klein 2006). 


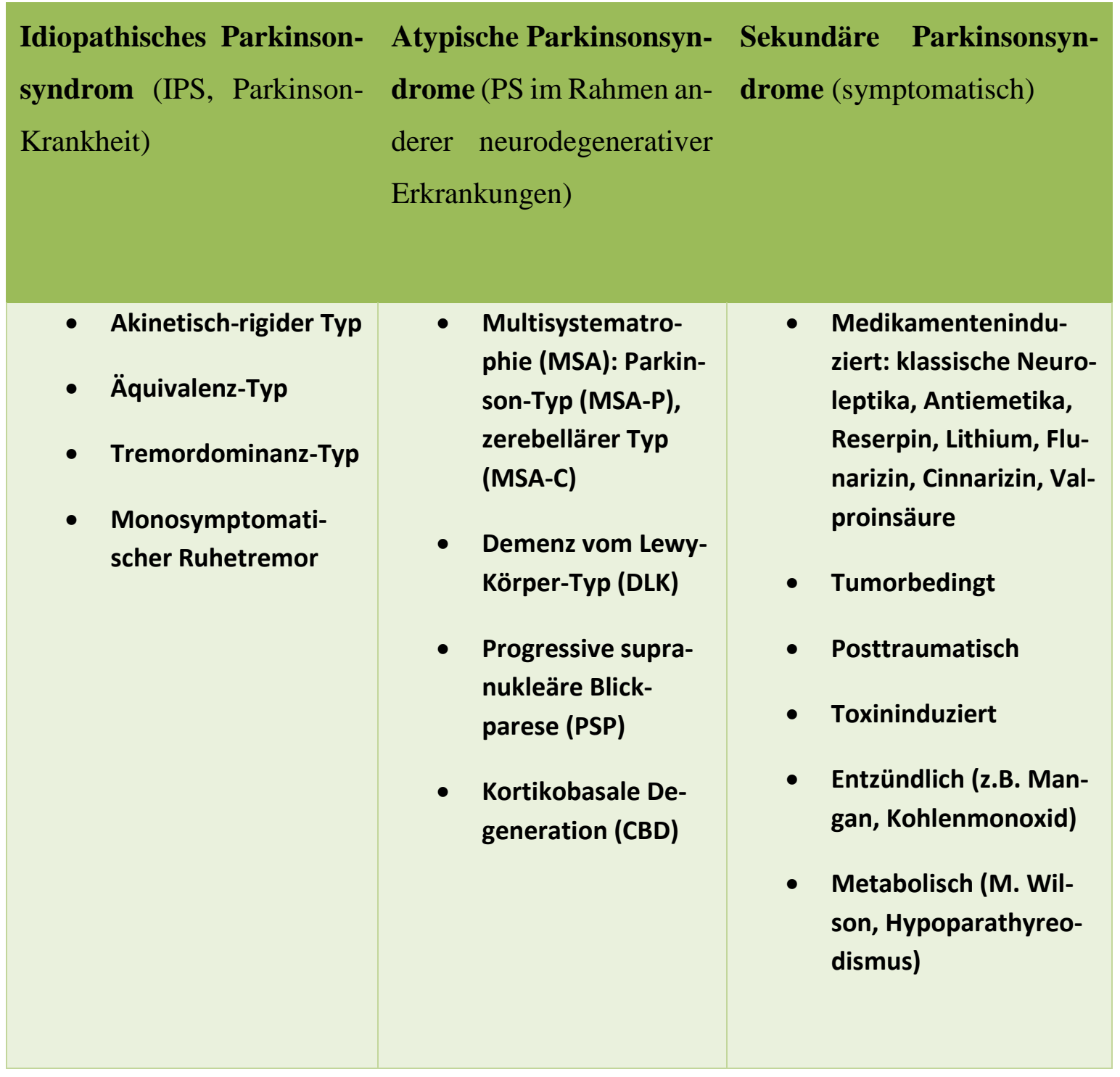

PS= Parkinsonsyndrom

Parkinson ähnliche Symptome können auch durch Toxine wie Phenothiazine ausgelöst werden. Die Symptome können Monate nach Exposition anhalten. MethylPhenyl-Tetrahydropyridine soll sogar eine schwere, anhaltende Parkinsonsymptomatik auslösen (Hawkes 1999).

Differenzialdiagnostisch muss ein vaskuläres Parkinsonsyndrom, ein Normaldruckhydrozephalus, ein essentieller Tremor und eine Depression berücksichtigt werden (Leitlinie idiopathisches Parkinson-Syndrom 2016). 


\subsection{Pathophysiologische Prozesse des IPS}

Das idiopathische Parkinsonsyndrom ist eine Erkrankung, bei der es frühzeitig zu einem Untergang dopaminerger Neurone in der Substantia nigra, Pars compacta, kommt. Die Abnahme des Dopamins in den Basalganglien führt daraufhin zu den für die Parkinsonerkrankung spezifischen motorischen Störungen (Kalia and Lang 2015). Der Verlust der Dopamin-produzierenden Neurone und die Entstehung von Lewy Körperchen stellen den entscheidenden pathologischen Befund dar (Connolly und Lang 2014). Man nimmt an, dass zum Zeitpunkt der Diagnosestellung bereits mehr als $60 \%$ der dopaminergen Neurone im nigrostriatalen System untergegangen sind (Adler 2011). Die bei der vorliegenden Erkrankung vorhandenen Lewy Körperchen und Lewy Neuriten bestehen aus abnorm aggregiertem $\alpha$-Synuklein (Kalia und Lang 2015). Das idiopathische Parkinsonsyndrom gehört deshalb zu der Gruppe der Synukleinopathien, zu der auch die Multisystematrophie (MSA) und die Lewy-Körper-Demenz zählt (Mollenhauer et al. 2011).

Im Gegensatz zur Substantia nigra, wo eine Abnahme der dopaminergen Neurone der Erkrankung zugrunde liegt, zeigte sich laut Huisman et al. (2004) im Bereich des Bulbus olfactorius eine Zunahme der dopaminergen Zellen.

Dabei gehen Braak et al. (2003) davon aus, dass die neuropathologischen Prozesse in 6 aufeinander folgenden Stadien ablaufen. Diese werden nachfolgend beschrieben. Die neuropathologischen Prozesse beginnen in den beiden präsymptomatischen Stadien 1 und 2 mit der Beteiligung der Medulla oblongata, des pontinen Tegmentums sowie dem Bulbus olfactorius und Nucleus olfactorius anterior. Im Stadium 3 breitet sich die Erkrankung bis in das Mittelhirn aus. Erst in diesem Stadium ist die Pars compacta der Substantia nigra beteiligt. Das Prosencephalon und der Mesocortex sind in Stadium 4 betroffen. Die Stadien 5 und 6 spiegeln die Einbeziehung des Neocortex wider.

Durch die im Laufe der Erkrankung entstehende Beteiligung nicht-dopaminerger Regionen des Gehirns entstehen motorische und non-motorische Symptome, die nicht auf L-Dopa ansprechen (Connolly und Lang 2014). Harding (2002) identifizierte beispielsweise beim idiopathischen Parkinsonsyndrom einen signifikanten Verlust des Volumens und der Neurone im Nucleus corticalis der Amygdala, assoziiert mit einer hohen Konzentration an Lewy Körpern. Der Nucleus corticalis der 
Amygdala ist ein Teil des olfaktorischen Systems und nimmt damit Einfluss auf die Riechfunktion (Swanson und Petrovich 1998).

Symptome, die auf das Vorliegen eines idiopathischen Parkinsonsyndroms hinweisend sein können, sind die idiopathische olfaktorische Dysfunktion (Sommer et al. 2004), REM-Schlaf-Verhaltensstörungen (Postuma und Montplaisir 2006), Obstipation (Spiegel et al. 2006) und Riechstörungen bei Verwandten ersten Grades der Parkinsonpatienten (Ponsen et al. 2004).

\subsection{Therapie des IPS}

Die derzeitige, ausschließlich symptomatische Behandlung der Erkrankung erfolgt durch Medikamente, die die Dopaminkonzentration steigern oder direkt Dopaminrezeptoren beeinflussen (Kalia und Lang 2015). Vor ca. 50 Jahren wurde die hochdosierte L-Dopa-Therapie eingeführt und ist nach wie vor der Goldstandard zur symptomatischen Behandlung des idiopathischen Parkinsonsyndroms (Fahn 2015). Systemische Wirkungen des Levodopa wie Übelkeit, Erbrechen und orthostatische Hypotension werden durch die zusätzliche Gabe von peripher wirksamen Decarboxylase-Inhibitoren, wie Carbidopa oder Benserazid, reduziert (Tarsy 2012). Diese werden grundsätzlich mit einer L-Dopa-Therapie kombiniert. Additiv können COMT (Catechol-O-Methyltransferase) -Inhibitoren, wie Entacapon oder Tolcapon, eingesetzt werden. Diese dienen der Verminderung motorischer Fluktuationen. Bei den Dopaminagonisten sollten ausschließlich Non-Ergot Präparaten wie Piribedil, Pramipexol, Ropinirol, Apomorphin und Rotigotin verschrieben werden. Diese können, ebenso wie L-Dopa-Präparate, in allen Stadien der Erkrankung verabreicht werden (DGN 2008). MAO (Monoaminoxidase)-B-Hemmer, wie Rasagilin und Selegilin, können des Weiteren in frühen Stadien der Erkrankung eingesetzt werden (Stern et al. 2004).

Bei anhaltenden Beschwerden trotz L-Dopa-Therapie in Bezug auf Stürze, posturale Instabilität und Ganginitiierungsstörungen, sogenanntes freezing, kann Amantadin (N-Methyl-D-Aspartat-Blocker) der Therapie hinzugefügt werden (Connolly und Lang 2014).

Die dopaminerge Therapie kann im Verlauf zu motorischen Fluktuationen und Dyskinesien führen, was unter anderem eine Indikation für ein stereotaktisches 
Operationsverfahren wie die Tiefenhirnstimulation darstellen kann. Die Tiefenhirnstimulation beeinflusst über Elektroden den Nucleus subthalamicus, wodurch eine kontinuierliche Beeinflussung der Symptome ohne motorische Fluktuationen, die durch einen schwankenden Plasmaspiegel des L-Dopa entstehen können, gewährleistet werden kann (Kleiner-Fisman et al. 2006). Eine Heilungschance besteht jedoch durch diese Therapie nicht (Sixel-Döring und Ebersbach 2010).

Nach der derzeitigen wissenschaftlichen Lage zu urteilen, ist es unwahrscheinlich, dass eine medikamentöse Therapie die Progression der Erkrankung in einem fortgeschrittenen Stadium aufhalten könnte. Deshalb wäre es für die zu entwickelnde neuroprotektive Therapie ausschlaggebend, die Erkrankung in frühen Stadien diagnostizieren zu können (Becker et al. 2002). Ziel der derzeitigen Forschung ist es demnach, Biomarker zu finden, die die Risikogruppe für das idiopathische Parkinsonsyndrom definieren (Adler 2011). Eine Diagnose in der präklinischen Phase könnte darüber hinaus weitere Erkenntnisse über die neurodegenerativen Prozesse ermöglichen (Berendse et al. 2001). 


\section{Material und Methoden}

\subsection{Die DeNoPa-Kassel-Studie}

Die De-Novo-Parkinson-Kassel-Studie ist eine prospektive Langzeit-Follow-up-Studie (Mollenhauer et al. 2013), die unter der Studienleitung von Prof. Dr. med. Brit Mollenhauer und Prof. Dr. med. Claudia Trenkwalder in der Paracelsus-Elena-Klinik in Kassel initiiert wurde. Mit dem Ziel der verbesserten Früherkennung der Parkinsonerkrankung begann die Studie im September 2009. Bis 2012 konnten 159 Parkinsonpatienten und 110 gesunde Probanden für die Studie geworben werden. Die Baseline-Erfassung der Daten erfolgte 2013, im Anschluss daran erfolgten im Abstand von jeweils circa einem Jahr Folgeuntersuchungen (Follow-up). Nach dem Stand der letzten Folgeuntersuchung 2015 erfüllten für die vorliegende statistische Auswertung noch 117 Patienten und 100 Kontrollpersonen die Einschlusskriterien der Studie. Von den ursprünglich 159 Patienten nahmen 13 Personen nicht mehr an den Folgeuntersuchungen teil, 5 Personen verstarben bis zum Zeitpunkt des letzten Follow-ups. Bei 24 Patienten wurde in der Zwischenzeit eine andere neurologische Diagnose gestellt. Von den Kontrollpersonen erschienen insgesamt 5 Personen nicht mehr zu der Folgeuntersuchung, 4 verstarben und eine Person entwickelte einen Tremor. Demnach verblieben nach zwei Jahren noch 100 Kontrollpersonen.

Die Genehmigung der DeNoPa-Studie durch die Ethik-Kommission der Landesärztekammer Hessen erfolgte im März 2009.

Für den Einschluss in die DeNoPa-Studie musste bei der Patientengruppe zunächst die Verdachtsdiagnose des idiopathischen Parkinsonsyndroms gestellt werden. Es mussten dafür mindestens zwei der drei Kardinalsymptome Rigor, asymmetrischer Ruhetremor und Bradykinese vorliegen. Zudem musste im Levodopa-Test zu Beginn der Studie eine positive Auswertung vorliegen. Es durfte keine Therapie vorangegangen sein, die den Dopaminspiegel beeinflusst, oder die Therapie musste für mindestens vier Wochen vor Beginn der Datenerhebung abgesetzt werden. Des Weiteren durfte kein Verdacht auf ein atypisches Parkinson-Syndrom bestehen, das sich beispielsweise durch häufige Stürze oder eine Blickparese abzeichnen kann. Ausschlusskriterium war außerdem eine vaskuläre Enzephalopathie oder andere 
morphologische Veränderungen, die durch ein MRT belegt sind. Alle Probanden mussten ein Alter zwischen 40 und 85 Jahren aufweisen.

Die Probanden der Kontrollgruppe durften keine neurologischen Erkrankungen aufweisen. Ausgeschlossen wurden ebenfalls alle Probanden, die sich aufgrund von Kontraindikationen einer MRT-Untersuchung nicht unterziehen dürfen.

Die nachfolgende Tabelle 4 zeigt die Testungen, die im Rahmen der DeNoPa-Studie durchgeführt wurden und in der vorliegenden Arbeit statistisch ausgewertet werden. In Kapitel 2.2 wird der Inhalt und die Durchführung der Testungen beschrieben. Tabelle 4 Testungen im Rahmen der DeNoPa-Kassel-Studie

\section{Neurologische Testungen \\ $>$ MDS UPDRS \\ $>$ Hoehn \& Yahr \\ Riechtestungen \\ $>$ persönliche Einschätzung \\ $>$ Schwelle, Diskrimination, Identifikation}

Fragebögen zu non-motorischen Symptomen

$>$ PD NMS-Fragebogen

$>$ Skala zu non-motorischen Symptomen

$>P D Q-39$

\section{Depression}

$>B D S$

$>G D I$

$>$ MADRS

\section{Polysomnographie}

Neuropsychologische Testungen

$>$ MMST

$>$ Uhrentest

$>M o C A$

Bildgebende Verfahren

$>$ DAT-SPECT

\subsection{Baseline-Datenerhebung}

Im Rahmen der Baseline-Testungen zu Beginn der Studie wurden zunächst demographische Daten der Probanden erhoben. Darüber hinaus wurden umfangreiche Testungen zu der olfaktorischen Funktion, Depression und Lebensqualität, Schlaf, Kognition und neurologische Testungen durchgeführt. Alle Testungen wurden an 
beiden Testgruppen durchgeführt. Ausschließlich die Bildgebung mittels DAT-Scan wurde nur bei den Parkinsonpatienten durchgeführt.

\subsubsection{Neurologische Testungen}

Die MDS-UPDRS ist eine überarbeitete Version der UPDRS, die den Aufbau aus den vier Abschnitten des UPDRS, kognitive Funktion, Aktivitäten des täglichen Lebens, motorische Untersuchung und Komplikationen der Behandlung, beibehält. Zusätzlich werden spezifische Probleme der Parkinsonpatienten erfasst und Probleme behoben, die in der Originalfassung vorhanden waren (Goetz et al. 2007). Hier wird Abschnitt I (Maximalpunktzahl: 52 Punkte) als Erfahrungen des täglichen Lebens bezogen auf non-motorische Symptome und Abschnitt II (Maximalpunktzahl: 52 Punkte) als Erfahrungen des täglichen Lebens durch motorische Symptome definiert. Abschnitt III (Maximalpunktzahl: 76 Punkte) beschreibt die motorische Untersuchung. Der Abschnitt IV, motorische Komplikationen, wurde in der DeNoPa-Kassel-Studie nicht erhoben. In Abschnitt I-III kann demnach eine maximale Punktzahl von 180 Punkten erreicht werden.

Die Hoehn \& Yahr-Klassifikation beschreibt die Schwere der motorischen Symptomatik beim idiopathischen Parkinsonsyndrom. Dabei geht die Stadieneinteilung von 0 (keine Symptome) bis Stadium 5 (bettlägerig). Seit 1987 wird die modifizierte Hoehn \& Yahr-Skala angewandt. Diese wird in der nachfolgenden Tabelle 5 beschrieben.

Tabelle 5 Modifizierte Hoehn\& Yahr-Klassifikation

\section{Symptome}

\begin{tabular}{|l|l|}
\hline Stadium 0 & Keine sichtbaren Krankheitszeichen \\
\hline Stadium 1 & Unilaterale Erkrankung \\
\hline Stadium 1,5 & $\begin{array}{l}\text { Unilaterale Erkrankung unter Einbezie- } \\
\text { hung der Körperachse }\end{array}$ \\
\hline Stadium 2 & $\begin{array}{l}\text { Beidseitige Erkrankung ohne Beein- } \\
\text { trächtigung des Gleichgewichtssinnes }\end{array}$ \\
\hline
\end{tabular}




\begin{tabular}{|c|c|}
\hline Stadium 2,5 & $\begin{array}{l}\text { milde beidseitige Erkrankung ohne Be- } \\
\text { einträchtigung der posturalen Stabilität } \\
\text { im Zugtest }\end{array}$ \\
\hline Stadium 3 & $\begin{array}{l}\text { - milde bis mäßige beidseitige Erkran- } \\
\text { kung } \\
\text { - leichte posturale Instabilität } \\
\text { - körperlich unabhängig }\end{array}$ \\
\hline Stadium 4 & $\begin{array}{l}\text { - schwere Behinderung } \\
\text { - Fähigkeit besteht ohne Hilfe zu gehen } \\
\text { und zu stehen }\end{array}$ \\
\hline Stadium 5 & $\begin{array}{l}\text { - rollstuhlpflichtig } \\
\text { - bettlägerig, sofern keine Hilfe vorhan- } \\
\text { den }\end{array}$ \\
\hline
\end{tabular}

\subsubsection{Riechtestungen}

\subsubsection{Subjektive Riechtestungen und Fragebögen zu non-motorischen Sympto- men (persönliche Einschätzung, PD NMS-Fragebogen Frage 2, Skala zu non-motorischen Symptomen Frage 28)}

Die Probanden wurden zunächst zu ihrer persönlichen Einschätzung ihres Geruchssinnes befragt. Dabei konnten sie sich zwischen den Abstufungen „normal“, „vermindert“ oder „erhöht“ entscheiden.

Um non-motorische Symptome des idiopathischen Parkinsonsyndroms zu erfassen, wurde der PD NMS-Fragebogen (Chaudhuri et al. 2006) durchgeführt. Der Fokus lag bei der vorliegenden Auswertung vor allem bei der Frage 2 des PD NMS Fragebogens, in der nach subjektiven Veränderungen oder einem Verlust des Riechens und Schmeckens innerhalb des letzten Monats gefragt wurde. Im Fragebogen wurde außerdem beispielsweise nach Stuhlinkontinenz, Schwierigkeiten beim 
Schlucken oder Problemen, sich an Dinge zu erinnern, gefragt. Auch der Gesamtbetrag der insgesamt 30 Fragen (maximale Punktzahl: 30 Punkte) wurde statistisch ausgewertet.

Die Skala zur Erfassung nicht-motorischer Symptome bei der Parkinsonerkrankung (Chaudhuri et al. 2007) umfasst Fragen in insgesamt 9 verschiedenen Bereichen, diese beinhalten Fragen zu kardiovaskulären Beschwerden/ Stürzen, Schlaf/ Müdigkeit, Stimmung/ Kognition, Wahrnehmungsprobleme/ Halluzinationen, Aufmerksamkeit/ Gedächtnis, Gastrointestinaltrakt, Miktion, Sexualfunktion, Verschiedenes. Frage 28 befasst sich speziell mit Veränderungen des Riechens und Schmeckens. Dabei wurde die Frage nach der Häufigkeit ( $0=$ keine, $1=$ selten, $<1$ / Woche, 2= gelegentlich, 1/ Woche, 3= häufig, mehrmals pro Woche, 4= sehr häufig (täglich oder ständig) und Schwere $(0=$ keine, $1=$ leichte Symptome vorhanden, die wenig Belastung verursachen, 2= mäßige Belastung, 3= schwer, erhebliche Belastung) der Beschwerden untergliedert. Es wurde außerdem ein Score, bei dem die Häufigkeit mit der Schwere multipliziert wurde (Maximalwert: 12 Punkte) und ein Summenscore für die insgesamt 30 vorhandenen Fragen (Maximalwert: 150 Punkte) erhoben.

\subsubsection{Objektive Riechtestungen (Schwellentest, Diskriminationstest, Identifikati- onstest)}

Im Anschluss an die subjektiven Riechtestungen erfolgten umfangreiche objektive Testungen zu Geruchsdefiziten der Probanden, die mithilfe der Sniffin' Sticks durchgeführt wurden. Die sogenannten Sniffin' Sticks sind stiftförmige Applikatoren, die einen bestimmten Geruchsstoff enthalten. Diese wurden erstmals 1997 durch Hummel et al. (1997) zur Erfassung von Riechstörungen eingeführt. Es wurden insgesamt drei Testungen zur Schwelle, Diskrimination und zur Identifikation durchgeführt. Dabei wurde linksseitig bzw. rechtsseitig nicht separat gemessen.

Für den Schwellentest wurden dem Probanden drei Sniffin' Sticks präsentiert. Einer der Stifte besitzt dabei einen Geruchsstoff, den der Proband erkennen sollte. Dabei wurde in aufsteigender Verdünnung des Geruchsstoffes vorgegangen. Die Wertung erfolgt, wenn der Proband die höchste Verdünnung (maximal 16-fache Verdünnung) zwei Mal korrekt benannt hat. 
Für den Diskriminationstest wurden ebenfalls drei Stifte präsentiert, dabei hatten zwei von drei Stiften den gleichen Duft. Der dritte Stift wies einen anderen Duft auf und musste erkannt werden. 16 verschiedene Tripletts wurden hierbei getestet. Die Summe der korrekt beantworteten Gerüche ergibt den Diskriminationswert. Laut Ponsen et al. (2009) ist der Diskriminationstest am effektivsten, um eine zukünftige Parkinsonerkrankung vorherzusagen.

Abschließend wurde der Identifikationstest durchgeführt. Hier wurde die Wahrnehmung und Benennung der verschiedenen Gerüche getestet. Vier Antwortmöglichkeiten standen dabei zur Auswahl. Insgesamt 16 verschiedene Duftstoffe wurden getestet. Die Summe aller korrekten Antworten wurde als Gesamtwert in die Auswertung mit einbezogen. Die einzelnen Testergebnisse der Testungen 1-16 wurden zudem ausgewertet und mit denen der Folgeuntersuchung im Jahr 2015 verglichen.

\subsubsection{TDI-Score}

Durch die in den objektiven Riechtestungen erhobenen Daten konnte der TDI (threshold, discrimination, identification) -Score berechnet werden. Dieser ermöglicht eine Unterscheidung zwischen einer physiologischen Riechfunktion (Normosmie), einer abgeschwächten Riechfunktion (Hyposmie) und dem Verlust der Riechfunktion (Anosmie). Der TDI-Score wird über die Summe des Diskriminationstests, des Schwellentests und des Identifikationstests ermittelt. Die Punktzahl 30 stellte die Grenze zur Hyposmie dar (Wolfensberger et al. 2000). Eine Punktzahl $\leq 15$ spricht für eine Anosmie, dementsprechend eine Punktzahl $\geq 30$ für eine Normosmie (Hummel et al. 2007).

\subsubsection{Depression und Lebensqualität}

Die Geriatrische Depressionsskala (GDS) ist ein Fragebogen, der 15 Fragen umfasst. Er wurde von Yesavage et al. (1982-1983) entworfen und kann auf eine Altersdepression oder eine depressive Stimmungslage des Patienten hinweisen. Die Maximalpunktzahl des Tests liegt bei 15 Punkten. Bei einer Punktzahl von 5-10 Punkten würde man von einer leichten Depression sprechen, ab 11 Punkten von einer schweren Depression.

Der Beck-Depressions-Inventar (BDI)-Test, der 1961 von Beck et al. entwickelt wurde, ist ein psychologischer Fragebogen, der die Schwere einer depressiven 
Symptomatik erfassen soll. Zu 21 Themen (z.B. empfundene Traurigkeit, Zukunftsperspektive) werden Aussagen formuliert, die zu einer aufsteigenden Schwere der Symptomatik passen. Der Proband muss unter vier möglichen Aussagen, diejenige auswählen, die am meisten zutrifft. Dabei spricht eine insgesamt erreichte Punktzahl $\leq 13$ Punkten dafür, dass der Patient keine Depression hat. Eine Punktzahl zwischen 14-19 weist auf eine leichte Depression, eine Punktzahl zwischen 20-28 auf eine mittelgradige und eine Punktzahl $>28$ auf eine schwere Depression hin.

Die Montgomery-Åsberg Depression Rating Scale (MADRS) (Montgomery und Asberg 1979) wird als Interview durchgeführt. Die Bewertung des Untersuchers wird dabei in zehn Bereiche unterteilt: sichtbare Traurigkeit, innere Spannung, berichtete Traurigkeit, Schlaflosigkeit, Appetitverlust, Gefühllosigkeit, Konzentrationsschwierigkeiten, pessimistische Gedanken, Untätigkeit und Selbstmordgedanken. Bei diesem Test kann ein Maximalwert von 60 Punkten erreicht werden. Laut Müller et al. (2000) lässt sich dabei eine schwere Depression ab einer Punktzahl $\geq 35$ feststellen. Eine leichte Depression lässt sich aus einer Punktzahl von 9-17 Punkten, eine mittelgradige aus einer Punktzahl von 18-34 schließen (Mittmann et al. 1997).

Einen weiteren Test, der als Selbstfragebogen durchgeführt wurde, stellt der Parkinson's Disease-Fragebogen 39 (Peto et al. 1998) dar. Er dient zur Hilfestellung bei der Einschätzung der Lebensqualität von Parkinsonpatienten. Dabei werden die Fragen in 8 Kategorien (Mobilität, Aktivitäten des Lebens, emotionales Wohlbefinden, Stigmatisierung, soziale Unterstützung, kognitive Funktionen, Kommunikation, körperliche Missempfindungen) unterteilt. Er umfasst 39 Fragen, die sich vor allem auf nötige Hilfestellungen und die Selbstständigkeit des Patienten beziehen. Eine maximale Punktzahl von 156 Punkten kann in diesem Fragebogen erzielt werden.

\subsubsection{Schlaf}

Bei allen Probanden der DeNoPa-Kassel Studie wurde im Schlaflabor der Paracelsus-Elena-Klinik in Kassel eine Polysomnographie durchgeführt und mithilfe dessen der REM-Schlaf beurteilt. Dabei wurden während des Schlafes Elektroenzephalogramme (EEG), Elektrokardiogramme (EKG), Messungen der Muskelspannung (EMG) und Elektrookulogramme (EOG) aufgezeichnet. Darüber hinaus wurde pulsoxymetrisch der Sauerstoffgehalt des Blutes, die Körpertemperatur, der Atemfluss und die Beinbewegungen gemessen. Anhand der Befunde wurde diagnostiziert, ob 
eine REM-Schlaf-Verhaltensstörung (RBD), eine RBE oder ein fehlender REMSchlaf vorliegt.

\subsubsection{Kognition}

Der Mini-Mental-State Test dient zur Früherkennung kognitiver Abbauprozesse (Folstein et al. 1975). Dabei werden Fragen bzw. Aufgaben gestellt, die die Orientierung, die Merkfähigkeit, die Aufmerksamkeit, das Rechnen und Schreiben, die Erinnerungsfähigkeit, das Lesen und die visuo-konstruktive Fähigkeit prüfen. Ab Werten $\leq 25$ von 30 maximalen Punkten besteht der starke Verdacht, dass bei dem Patienten eine Demenz vorliegt.

Der Uhrentest kann neben dem Mini-Mental-State Test ebenfalls zum Screening kognitiver Einschränkungen durchgeführt werden. Hierfür malt der Patient eine Uhr in einen vorgezeichneten Kreis und soll die Uhrzeit „10 nach 11“ einzeichnen. Der Score für die Beurteilung geht hier von 1 (= perfekt, Ziffern richtig eingezeichnet, zwei Zeiger, die die richtige Uhrzeit anzeigen), 2 (= leichte visuell-räumliche zum Beispiel Ziffern stehen auf dem Kopf), 3 (= fehlerhafte Uhrzeit bei erhaltener visuellräumlicher Darstellung zum Beispiel nur ein Zeiger), 4 (= mittelgradige visuell-räumliche Desorganisation, sodass ein korrektes Einzeichnen der Uhrzeit unmöglich ist zum Beispiel Ziffern vergessen) bis 5 (keinerlei Darstellung einer Uhr, keine entfernte Ähnlichkeit mit einer Uhr, Patient schreibt Worte oder den Namen).

Ein weiterer Test, um milde kognitive Störungen, inklusive Alzheimer, zu identifizieren, ist das Montreal Cognitive Assessment (MoCA). Dabei werden das Gedächtnis, die Aufmerksamkeit, die Sprache, abstraktes Denken, Orientierung und exekutive Funktionen getestet. Eine Punktzahl > 25 Punkten von 30 möglichen Punkten wird als normale kognitive Leistung gewertet.

\subsubsection{Bildgebende Verfahren}

Die Dopamintransporter-Einzelphotonen-Emissions-Computertomographie (DATSPECT) ist eine bestimmte Form der Szintigraphie, bei der die Funktionsfähigkeit der zerebralen Dopamin-Transporter dargestellt werden kann. Diese Untersuchung wurde nur bei den Patienten durchgeführt. 


\subsection{Folgeuntersuchungen}

Es fanden im Rahmen der DeNoPa-Kassel-Studie zwei Folgeuntersuchungen, nach 12 und nach 24 Monaten, statt. Dabei wurden erneut umfangreiche Testungen durchgeführt. Für die statistische Auswertung der vorliegenden Arbeit wurden die Daten der Folgeuntersuchungen nach 24 Monaten verwendet. Es wurden vergleichend für die Baseline- und Folgeuntersuchungen nach 24 Monaten die Daten der subjektiven Einschätzung des Riechvermögens und objektive Riechtestungen zur Identifikation, Diskrimination und Schwelle dargestellt. Des Weiteren wurden klinische Parameter wie polysomnographische Daten und Daten der neuropsychologischen Testungen verglichen.

\subsection{Statistische Analyse}

Die statistische Auswertung der vorliegenden Arbeit erfolgte mit IBM $\circledast$ SPSS $\circledast$ Statistics 22, STATISTICA® 10, Excel $\AA^{2} 2016$ und BiAS $\AA$. Der Text wurde in Microsoft Word® 2016 verfasst.

Zunächst erfolgte die deskriptive Darstellung der Daten mithilfe von Mittelwerten (M), der Standardabweichung (SD), der Anzahl (n) und der prozentualen Verteilungen (\%). Die graphische Darstellung der Daten wurde durch Box-plots, Kreisdiagramme und Balkendiagramme umgesetzt.

Die Berechnung der Signifikanz für unabhängige Variablen erfolgte je nach Skalierung der Daten mit dem Chi-Quadrat-Test, dem Mann-Whitney-U-Test und dem tTest. Bei nominal skalierten Daten wurde der Chi-Quadrat-Test, bei ordinal skalierten Daten der Mann-Whitney-U-Test und bei Intervallskalen der t-Test angewandt. Für abhängige Variablen (gleicher Test, zwei festgelegte Zeitpunkte) wurde der pWert bei nominal skalierten Daten durch den McNemar-Test und für ordinal skalierte Daten durch den Wilcoxon-Test berechnet. Der daraus errechnete $p$-Wert bestätigt bei einem Wert $<.05(<5 \%)$ die Verwerfung der Nullhypothese und die Annahme der Gegenhypothese. Daraus wird ein signifikanter Unterschied zwischen den verglichenen Daten angenommen, der nicht auf einen Zufall zurückzuführen ist.

Die in dieser Arbeit aufgeführten Korrelationen entsprechen der Korrelation nach Spearman, die einen linearen Zusammenhang zwischen zwei Werten zeigen kann. Die Konvention nach Cohen legt für den Korrelationskoeffizienten ( $r$ ) einen Bereich 
von $-1 \leq r \leq+1$ fest. Der Korrelationskoeffizient beschreibt dabei die Enge des Zusammenhangs zwischen zwei Variablen. Ist $r>0$, so spricht man von einem positiven Zusammenhang (je größer die eine Variable ist, desto größer wird auch die andere), ist $r<0$, so bedeutet dies, dass ein negativer Zusammenhang besteht (je größer die eine Variable ist, umso kleiner wird die andere). $R=0$ zeigt, dass kein Zusammenhang besteht. Ein vollständig, linearer, positiver Zusammenhang wäre bei $r=+1$ gegeben, dementsprechend ein vollständig, linearer, negativer Zusammenhang bei -1. Ab einem Korrelationskoeffizienten von 0,1 würde man den Zusammenhang als geringgradig bezeichnen, ab 0,3 als mittelgradig und ab 0,5 als hochgradig.

Zur Bewertung diagnostischer Tests wird die Sensitivität und die Spezifität zu Hilfe genommen. Dabei zeigt die Sensitivität, wie viele Kranke von einem diagnostischen Test auch als „krank“ erkannt werden. Die Sensitivität ist demzufolge definiert als die Rate „richtig positiver" Ergebnisse. Dem gegenüber steht die Spezifität. Diese beschreibt, wie viele Gesunde von einem diagnostischen Test als „gesund“, also als „richtig negativ" bestätigt werden.

Tabelle 6 Sensitivität und Spezifität

\begin{tabular}{|l|l|l|}
\hline Diagnose & Testergebnis positiv & Testergebnis negativ \\
\hline krank & richtig positiv & falsch negativ \\
\hline gesund & falsch positiv & richtig negativ \\
\hline
\end{tabular}

Die ROC (receiver operating characteristic)- Kurve liefert einen visuellen Eindruck der "Güte" eines Diagnostischen Tests. Dabei wird die Fläche unter der Kurve (area under the curve) als Maß genommen.

Der Youden-Index ist ein Maß zur Beurteilung der Güte eines diagnostischen Tests, dessen wichtigste Aussagekraft die Kennzeichnung der Erkrankten in einem Test ist (Youden 1950). Er wird berechnet aus der Sensitivität und Spezifität (Sensitivität - Spezifität -1). Er kann Werte zwischen -1 und +1 annehmen. Bei einem diagnostischen Test sollte ein Youden-Index zwischen 0 und +1 erreicht werden. Je näher der Youden-Index bei +1 liegt, desto besser ist der vorliegende diagnostische Test in der Lage, Kranke und Gesunde voneinander zu trennen. 


\section{ERgeBNISSE}

\subsection{Demographische Daten}

In die statistische Auswertung wurden 117 Patienten mit einem idiopathischen Parkinsonsyndrom sowie 100 Kontrollpersonen einbezogen. Weitere demographische Daten zeigt Tabelle 7.

Tabelle 7 Demographische Daten, MDS UPDRS und Hoehn \& Yahr-Klassifikation der Parkinsonpatienten (PD) und der gesunden Kontrollgruppe (HC) für die Baseline-Testung und die Folgetestung nach 24 Monaten und der darauf bezogene $\mathrm{p}$-Wert

\begin{tabular}{|c|c|c|c|c|c|c|}
\hline \multirow{2}{*}{$\begin{array}{l}\text { Demographische } \\
\text { Daten }\end{array}$} & \multicolumn{2}{|c|}{ PD } & \multicolumn{2}{|c|}{$\mathrm{HC}$} & \multirow{2}{*}{$\begin{array}{l}\text { p- } \\
\text { Wert, } \\
\text { BL PD } \\
\text { vs. } \\
\text { HC }\end{array}$} & \multirow{2}{*}{$\begin{array}{l}\text { p- } \\
\text { Wert, } \\
\text { FU PD } \\
\text { vs. } \\
\text { HC }\end{array}$} \\
\hline & BL & FU & BL & FU & & \\
\hline Alter M (SD) & $\begin{array}{l}(\mathrm{n}=117) \\
63,67 \\
(9,63)\end{array}$ & $\begin{array}{l}(n=117) \\
67,76 \\
(9,61)\end{array}$ & $\begin{array}{l}(n=100) \\
64,5 \\
(6,85)\end{array}$ & $\begin{array}{l}(n=100) \\
68,68 \\
(6,83)\end{array}$ & .460 & .452 \\
\hline $\begin{array}{l}\text { Geschlecht } \\
\text { (Männlich/ weib- } \\
\text { lich, \%) }\end{array}$ & $\begin{array}{l}(\mathrm{n}=117) \\
76 / 41 \\
(65 \% / 35 \%)\end{array}$ & - & $\begin{array}{l}(n=100) \\
60 / 40 \\
(60 \% / 40 \\
\%)\end{array}$ & - & .452 & - \\
\hline $\begin{array}{l}\text { Dauer des Schul- } \\
\text { besuches (Jahre, } \\
\text { M) }\end{array}$ & $\begin{array}{l}(n=117) \\
9,44(1,70)\end{array}$ & - & $\begin{array}{l}(n=100) \\
9,64 \\
(1,81)\end{array}$ & - & .553 & - \\
\hline
\end{tabular}




\begin{tabular}{|c|c|c|c|c|c|c|}
\hline $\begin{array}{l}\text { Hoehn\& Yahr M } \\
\text { (SD) }\end{array}$ & $\begin{array}{l}(n=117) \\
1,76(0,64)\end{array}$ & $\begin{array}{l}(\mathrm{n}=116) \\
2,15 \\
(0,82)\end{array}$ & $\begin{array}{l}(n=100) \\
0,00 \\
(0,00)\end{array}$ & $\begin{array}{l}(n=100) \\
0,00 \\
(0,00)\end{array}$ & $<.001$ & $<.001$ \\
\hline $\begin{array}{l}\text { MDS UPDRS I M } \\
\text { (SD) }\end{array}$ & $\begin{array}{l}(n=117) \\
5,98(4,21)\end{array}$ & $\begin{array}{l}(n=116) \\
12,91 \\
(7,22)\end{array}$ & $\begin{array}{l}(n=100) \\
2,48 \\
(2,41)\end{array}$ & $\begin{array}{l}(n=100) \\
5,41 \\
(4,18)\end{array}$ & $<.001$ & $<.001$ \\
\hline $\begin{array}{l}\text { MDS UPDRS II M } \\
\text { (SD) }\end{array}$ & $\begin{array}{l}(n=117) \\
8,06(4,84)\end{array}$ & $\begin{array}{l}(n=116) \\
13,49 \\
(8,02)\end{array}$ & $\begin{array}{l}(n=100) \\
0,16 \\
(0,49)\end{array}$ & $\begin{array}{l}(n=100) \\
1,24 \\
(1,57)\end{array}$ & $<.001$ & $<.001$ \\
\hline $\begin{array}{l}\text { MDS UPDRS III M } \\
\text { (SD) }\end{array}$ & $\begin{array}{l}(n=117) \\
21,97 \\
(11,67)\end{array}$ & $\begin{array}{l}(n=116) \\
30,10 \\
(14,76)\end{array}$ & $\begin{array}{l}(n=100) \\
0,51 \\
(1,31)\end{array}$ & $\begin{array}{l}(n=100) \\
1,40 \\
(2,49)\end{array}$ & $<.001$ & $<.001$ \\
\hline $\begin{array}{l}\text { MDS UPDRS Ge- } \\
\text { samt M (SD) }\end{array}$ & $\begin{array}{l}(n=117) \\
36,05 \\
(17,62)\end{array}$ & $\begin{array}{l}(n=116) \\
57,67 \\
(26,39)\end{array}$ & $\begin{array}{l}(n=100) \\
3,15 \\
(2,97)\end{array}$ & $\begin{array}{l}(n=100) \\
8,05 \\
(6,89)\end{array}$ & $<.001$ & $<.001$ \\
\hline
\end{tabular}

$\mathrm{PD}=$ Parkinsonpatienten, $\mathrm{HC}=$ (healthy control) Kontrollgruppe, $\mathrm{n}=$ Anzahl, $\mathrm{M}=$ Mittelwert, $\mathrm{SD}=$ Standardabweichung

Für das Alter, das Geschlecht und die Dauer des Schulbesuchs zeigten sich keine signifikanten Unterschiede. Im Mittel lag das Alter der Patienten bei der BaselineDatenerfassung bei 63,67 Jahren, das der Kontrollgruppe bei 67,76 Jahren. Die Geschlechterverteilung lag bei 65 \% Männer und $35 \%$ Frauen bei den Patienten, im Vergleich zu der Kontrollgruppe mit 60 \% Männern und 40 \% Frauen. Mit im 
Mittel 9,44 Schuljahren bei den Patienten und 9,64 Jahren in der Kontrollgruppe zeigte sich auch hier ein ausgeglichenes Bild.

\subsubsection{Hoehn \& Yahr-Klassifikation}

25,6 \% (30 Personen) der Patienten waren beim Einschluss in die Studie im Stadium 1 nach Hoehn \& Yahr, 31,6 \% (37 Personen) im Stadium 1,5, 16,2 \% (19 Personen) im Stadium 2, 17,9 \% (21 Personen) im Stadium 2,5 und 8,5 \% (10 Personen) im Stadium 3. Im Stadium 4 und 5 befanden sich zum Zeitpunkt der Erhebung keine Patienten. Bei der Auswertung lag von allen 117 Patienten eine Angabe vor. In der Folgeuntersuchung zeigten sich $19 \%$ der Patienten (22 Personen) im Stadium 1, 8,6 \% (10 Personen) im Stadium 1,5, 33,6 \% (39 Personen) im Stadium 2, 15,5 \% (18 Personen) im Stadium 2,5, 16,4 \% (19 Personen) im Stadium 3 und 6,9 \% (8 Personen) im Stadium 4. Der Unterschied zwischen der Patientengruppe und der Kontrollgruppe erwies sich sowohl in der Baseline- als auch in der Folgeuntersuchung als signifikant $(p<.001)$.

\subsubsection{MDS-UPDRS I-III und Gesamt}

Im Mittel erreichten die Patienten (Minimalwert der Patienten $=0$, Maximalwert der Patienten =19 Punkte) in der MDS UPDRS I Klassifikation eine Punktzahl von 5,98, die Kontrollgruppe (Minimalwert der Kontrollgruppe $=0$, Maximalwert der Kontrollgruppe =15 Punkte) 2,48 Punkte.

Im Abschnitt II des MDS UPDRS zeigten sich Mittelwerte von 8,06 Punkten (Min. $=0$, Max. $=29$ Punkte) bei den Patienten und bei den Kontrollpersonen 0,16 bei Maximalwerten von 2 Punkten und Minimalwerten von 0 Punkten. Abschnitt III zeichnete sich durch einen Mittelwert von 21,97 Punkten bei den Patienten (Min. =3, Max. =66 Punkte) und einen Mittelwert von 0,51 Punkten (Min. =0, Max. =6 Punkte) bei der Kontrollgruppe aus. Für die Gesamtpunktzahl der Testabschnitte I-III ergaben sich bei den Patienten Mittelwerte von 36,05 Punkten (Min. =7, Max. =88), bei den Kontrollpersonen 3,15 Punkte (Min. =0, Max. =15). Ein signifikanter Unterschied konnte in allen vier Tests zwischen der Patientengruppe und den Kontrollpersonen nachgewiesen werden $(p<.05)$. 
Die Berechnungen zu der MDS-UPDRS ergaben bei den Patienten im Mittel höhere Punktzahlen als in der Kontrollgruppe. Demzufolge zeigte die Testung bei den Patienten eine stärkere Ausprägung motorischer Ausfälle, Einschränkungen im Alltag und non-motorischer Symptome auf als bei der Kontrollgruppe.

\subsubsection{Dauer der Erkrankung nach Erstmanifestation}

Die Zeitspanne nach dem Auftreten des ersten motorischen Symptoms lag bei den Patienten zum Zeitpunkt der Baseline Testungen im Mittel bei 23,7 Monaten, wobei die minimale Anzahl zwei und die maximale Anzahl 240 Monate betrug.

\section{Die Dauer der Erkrankung nach Auftreten der ersten Symptome bei den Patienten zum Zeitpunkt der Baseline-Testung}

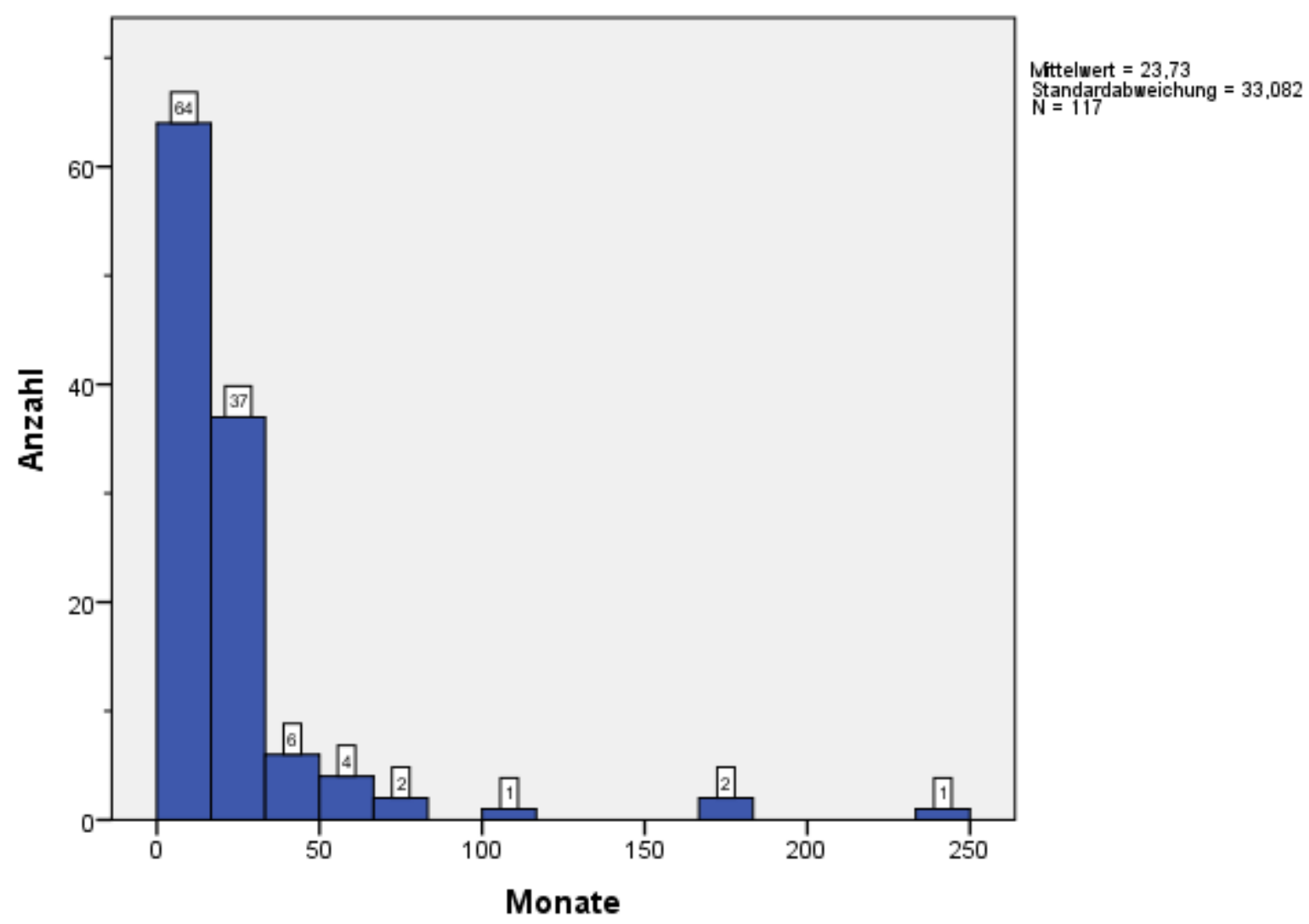

Abbildung 1 Dauer der Erkrankung der Parkinsonpatienten

Anamnestisch gaben 100 Kontrollpersonen und 114 Patienten an weitere Erkrankungen zu haben. Lediglich drei Patienten hatten keine weiteren Erkrankungen. Des Weiteren wurde bei den Patienten vor Einschluss in die Studie ein DAT-Scan durchgeführt. Von den dabei getesteten 99 Patienten zeigten alle einen pathologischen Befund (100\%). 


\subsection{Klinische Parameter}

Tabelle 8 Klinische Parameter: Testungen zu Depression (BDI, GDS, MADRS), der Lebensqualität (PDQ-39), REM-Schlafstörungen (Polysomnographie) und Kognition (MMST, Uhrentest, MoCA) der Parkinsonpatienten (PD) und der Kontrollgruppe (HC), vergleichend dargestellt für die Baseline- und Folgetestung nach 24 Monaten

\begin{tabular}{|c|c|c|c|c|c|c|}
\hline \multirow{4}{*}{ Klinische Parameter } & \multicolumn{2}{|c|}{ PD } & \multicolumn{2}{|c|}{$\mathrm{HC}$} & \multirow{4}{*}{$\begin{array}{l}\text { p-Wert } \\
\text { BL } \\
\text { PD vs. } \\
\text { HC }\end{array}$} & \multirow{4}{*}{$\begin{array}{l}\text { p-Wert } \\
\text { FU } \\
\text { PD vs. } \\
\text { HC }\end{array}$} \\
\hline & \multirow[t]{3}{*}{ BL } & \multirow[t]{3}{*}{ FU } & \multirow[t]{3}{*}{$\mathrm{BL}$} & \multirow[t]{3}{*}{ FU } & & \\
\hline & & & & & & \\
\hline & & & & & & \\
\hline \multirow[t]{3}{*}{ PDQ- 39 M (SD) } & $(n=113)$ & $(n=115)$ & $(n=96)$ & $(n=99)$ & \multirow[t]{3}{*}{$<.001$} & \multirow[t]{3}{*}{$<.001$} \\
\hline & 27,59 & 34,10 & 8,62 & 9,61 & & \\
\hline & $(18,14)$ & $(24,89)$ & $(9,68)$ & $(10,75)$ & & \\
\hline \multirow[t]{3}{*}{ GDS M (SD) } & $(n=113)$ & $(n=113)$ & $(\mathrm{n}=98)$ & $(n=100)$ & \multirow[t]{3}{*}{$<.001$} & \multirow[t]{3}{*}{$<.001$} \\
\hline & 3,52 & 2,75 & 1,14 & 1,92 & & \\
\hline & $(3,13)$ & $(2,61)$ & $(1,52)$ & $(2,02)$ & & \\
\hline \multirow[t]{3}{*}{ BDI M (SD) } & $(n=114)$ & $(n=112)$ & $(n=100)$ & $(\mathrm{n}=98)$ & \multirow[t]{3}{*}{$<.001$} & \multirow[t]{3}{*}{$<.001$} \\
\hline & 8,74 & 7,76 & 3,54 & 3,62 & & \\
\hline & $(6,64)$ & $(5,96)$ & $(4,06)$ & $(4,30)$ & & \\
\hline \multirow[t]{3}{*}{ MADRS M (SD) } & $(n=110)$ & $(n=114)$ & $(n=100)$ & $(\mathrm{n}=96)$ & \multirow[t]{3}{*}{$<.001$} & \multirow[t]{3}{*}{$<.001$} \\
\hline & 8,82 & 3,61 & 3,96 & 1,78 & & \\
\hline & $(7,22)$ & $(4,58)$ & $(4,87)$ & $(4,69)$ & & \\
\hline \multirow{5}{*}{$\begin{array}{l}\text { PSG REM- } \\
\text { Schlafstörungen } \\
\text { Ohne patholog. Be- } \\
\text { fund/ RBD/ RBE/ } \\
\text { kein REM-Schlaf (\%) }\end{array}$} & $(n=117)$ & $(n=111)$ & $(n=100)$ & $(\mathrm{n}=92)$ & \multirow[t]{5}{*}{$<.001$} & \multirow[t]{5}{*}{$<.001$} \\
\hline & $59 / 30 / 27 /$ & $33 / 59 / 11 /$ & $83 / 2 / 15 /$ & $79 / 2 / 11 /$ & & \\
\hline & 1 & 8 & 0 & 0 & & \\
\hline & $(50,4 / 25,6$ & $(29,7 / 53$ & $(83 / 2 / 15$ & $(85,9 / 2$ & & \\
\hline & $/ 23,1 / 0,9)$ & $2 / 9,9 / 7,2)$ & /0) & 2/12/0) & & \\
\hline MMST M (SD) & $(n=116)$ & $(n=116)$ & $(\mathrm{n}=97)$ & $(n=100)$ & .062 & $<.001$ \\
\hline
\end{tabular}




\begin{tabular}{|c|c|c|c|c|c|c|}
\hline & 28,57 & 27,66 & 28,88 & 28,73 & & \\
& $(1,31)$ & $(2,46)$ & $(1,13)$ & $(1,53)$ & & \\
\hline & $(n=116)$ & $(n=117)$ & $(n=99)$ & $(n=100)$ & .001 & .018 \\
\hline Uhrentest M (SD) & 1,60 & 1,72 & 1,27 & 1,41 & & \\
\hline MoCA M (SD) & $(0,91)$ & $(1,00)$ & $(0,65)$ & $(0,73)$ & & \\
\hline & $(n=88)$ & $(n=116)$ & $(n=89)$ & $(n=100)$ & .038 & .004 \\
\hline & 25,15 & 24,49 & 26,01 & 26,12 & & \\
& $(2,83)$ & $(3,99)$ & $(2,39)$ & $(2,67)$ & & \\
\hline
\end{tabular}

PSG= Polysomnographie, REM= Rapid-Eye-Movement, $M=$ Mittelwert, SD= Standardabweichung

\subsubsection{Lebensqualität}

Im Parkinson's Disease Fragebogen (PDQ-39) konnte insgesamt eine maximale Anzahl von 100 Punkten erreicht werden. Im Mittel erreichten die Patienten in den Baseline-Testungen 27,59 Punkte. In der Folgeuntersuchung zeigte sich eine Progredienz hinsichtlich der Abnahme der Lebensqualität mit einem Mittelwert von 34,10 Punkten. Die Kontrollgruppe zeigte mit einem Mittelwert von 8,62 Punkten eine wesentlich geringer empfundene Einschränkung der Lebensqualität. Auch hier zeigte sich in der Folgeuntersuchung mit 9,61 Punkten eine Verschlechterung des Ausgangswertes.

\subsubsection{Depression}

Anamnestisch gaben zum Zeitpunkt der Datenerhebung 23 (19,7 \%) von 117 Patienten Depressionen in der Vorgeschichte an, während es bei den Kontrollpersonen $15(15 \%)$ waren.

Die maximal zu erreichende Punktzahl in der Geriatrischen Depressionsskala (GDS) liegt bei 15 Punkten. Im Mittel erreichten die Patienten 3,52 Punkte, die Kontrollgruppe 1,14 Punkte. Dabei wurden von 113 gezählten Antworten 35 Patienten als leicht depressiv bewertet, fünf Patienten als schwer depressiv. Von der Kontrollgruppe wurde keiner als schwer depressiv eingestuft, leicht depressiv waren vier Personen, von insgesamt 98 gezählten Antworten. Im Vergleich zeigten demnach mehr Patienten eine leichte Depression als Kontrollpersonen. Der $p$-Wert lag bei < 
.001, was auf einen signifikanten Unterschied hinweist. Diese Signifikanz zeigte sich auch in der Folgeuntersuchung, bei der statistisch ein $p$-Wert von $<.001$ ermittelt werden konnte. Die Mittelwerte nahmen in beiden Testgruppen ab.

Die Patienten erreichten im Beck-Depressions-Inventar Test in der Baseline-Testung einen Minimalwert von 0 Punkten und einen Maximalwert von 29,4 Punkten. Die Kontrollgruppe erreichte im Vergleich dazu einen Maximalwert von 25 Punkten (Min. =0 Punkte). Der Mittelwert lag bei den Patienten bei 8,74 Punkten von 114 gültigen Antworten, die Kontrollgruppe erreichte im Mittel 3,54 Punkte bei 100 ausgewerteten Antworten. Laut Test zeigten in der Baseline-Testung demnach $89 \mathrm{~Pa}$ tienten keine Depression, 17 Patienten eine leichte Depression, 7 Patienten eine mittelgradige Depression und ein Patient eine schwere Depression. In der Kontrollgruppe wiesen 97 Probanden keine Depression auf. Lediglich zwei Kontrollpersonen wiesen eine leichte Depression auf, eine mittelgradige Depression eine Person und eine schwere Depression keiner der Probanden. In der Folgeuntersuchung wiesen von den Patienten 96 keine Depression auf, 11 Patienten eine leichte und 5 Patienten eine mittlere Depression. Eine schwere depressive Erkrankung lag bei keinem Probanden vor. Bei den Kontrollen zeigten von 98 getesteten Probanden, 95 keine Anzeichen einer Depression, 2 zeigten eine leichte und eine Person eine mittlere Depression.

Bei der Montgomery-Åsberg Depression Rating Scale (MADRS) konnte ein Maximalwert von 60 Punkten erreicht werden, die Patienten erreichten im Mittel eine Punktzahl von 8,82 Punkten bei einem Maximalwert von 34 Punkten und einem Minimalwert von 0 Punkten. Der Mittelwert bei der Kontrollgruppe lag bei 3,96 Punkten (Max. $=26$ Punkte, Min. $=0$ Punkte). Keine depressive Symptomatik zeigten 69 Patienten, eine leichtgradige Depression zeigten hier 29 Patienten, eine mittelgradige 12 Patienten. Eine schwere Depression lag bei keinem der Probanden vor. 86 Kontrollpersonen zeigten hier keine Depression, 12 eine leichte und 2 eine mittelgradige Depression. Es zeigte sich ein signifikanter Unterschied zwischen den Gruppen, sowohl in der Baseline als auch in der Folgeuntersuchung bei $p<.05$. 


\subsubsection{Schlaf}

Bei den Studienteilnehmern wurde in der Polysomnographie untersucht, ob REMSchlafstörungen vorliegen. Dabei wurde abgestuft nach $0=R E M-S c h l a f$ ohne pathologischen Befund, 1 =REM-Schlaf-Verhaltensstörung (RBD), 2 =RBE, 3 =fehlender REM-Schlaf.

50,4 \% (59 Personen) der Patienten hatten einen REM- Schlaf ohne pathologischen Befund, 25,6 \% (30 Personen) eine REM-Schlaf Störung, 23,1 \% (27 Personen) wiesen ein RBE auf und bei 0,9\% (1 Person) fehlte der REM-Schlaf. Bei der Kontrollgruppe hatten 83 \% (83 Personen) einen normalen REM-Schlaf, 2 \% (2 Personen) hatten eine REM- Schlafstörung und $15 \%$ (15 Personen) Personen ein RBE.

49,57\% der befragten Patienten zeigten in der Polysomnographie Schlafstörungen. Im Vergleich waren es bei den Kontrollpersonen nur $17 \%$, womit der Test zeigt, dass bei den Parkinsonpatienten eine wesentlich höhere Anzahl an Personen Schlafstörungen aufweisen.

\subsubsection{Kognition}

Bei dem Mini-Mental-State Test, der als Screening für kognitive Einschränkungen eingesetzt wird, kann eine maximale Punktzahl von 30 Punkten erreichten werden. Eine Punktzahl unter 25 Punkten weist auf eine Demenz hin. Hier zeigte sich kein signifikanter Unterschied zwischen den verglichenen Gruppen $(p=.062)$. Bei den Patienten erzielte nur eine Person mit 22 Punkten einen Wert unter 25 Punkten. Demzufolge wird nur bei einem Patienten der Verdacht einer Demenz Erkrankung geäußert. Alle anderen Testpersonen lagen über 25 Punkten. Hingegen zeigte sich in der Folgeuntersuchung ein signifikanter Unterschied. Der Mittelwerte der Parkinsonpatienten nahm in der Folgeuntersuchung ab.

Der Uhrentest stellte sich hier als signifikant dar $(p<.05)$.

Für die Patienten zeigte sich im Montreal Cognitive Assessment (MoCA) ein Mittelwert von 25,15 Punkten (Min. =16, Max. =30 Punkte), für die Kontrollgruppe ein Mittelwert von 26,01 Punkten (Min. =19, Max. =30 Punkte). Es zeigte sich bei den verglichenen Gruppen ein signifikanter Unterschied für diesen Test $(p<.05)$. 
37 Patienten erzielten Punktzahlen unter 25 und zeigten in diesem Test eine verminderte kognitive Leistung an. Bei den Kontrollpersonen erreichten 18 Personen weniger als 25 Punkte.

\subsection{Riechtestungen}

Die Riechtestungen wurden untergliedert in subjektive und objektive Riechtestungen. Die subjektiven Riechtestungen umfassen die persönliche Einschätzung, die Frage 2 des Parkinson's Disease Non-Motor Symptoms Fragebogens und deren Gesamtwertung sowie die Frage 28 in der Skala zu non-motorischen Symptomen mit der Untergliederung in Häufigkeit und Schwere der Symptome und deren Summenwert. Die objektiven Riechtestungen wurden mittels Sniffin`Sticks durchgeführt und wurden in die Bereiche Identifikationstest, Diskriminationstest und Schwellentest unterteilt.

\subsubsection{Subjektive Bewertungen des Riechvermögens}

In allen Testungen zu den subjektiven Beschwerden zeigten sich hoch signifikante Unterschiede zwischen der Kontrollgruppe und den Parkinsonpatienten.

Tabelle 9 Subjektive Bewertung des Riechvermögens: Persönliche Einschätzung, Befragung durch PD NMS-Fragebogen Frage 2, Skala zu non-motorischen Symptomen Frage 28 und deren Signifikanz ( $p-$ Werte)

\begin{tabular}{|l|l|l|l|}
\hline & PD & HC & p-Wert \\
\hline Persönliche Einschätzung & $(n=115)$ & $(n=100)$ & $<.001$ \\
\hline normal/vermindert/erhöht & $41 / 74 / 0$ & $79 / 13 / 8$ & \\
& $(35,65 \% /$ & $(79 \% / \quad 13 \% /$ & \\
\hline PD NMS-Fragebogen Frage 2 & $(n=117)$ & $(n=100)$ & $<.001$ \\
\hline ja/nein & $72 / 45(61,5 \% /$ & $10 / 90(10 \% /$ & \\
\hline PD NMS-Fragebogen Gesamt & $(n=117)$ & $90 \%)$ & \\
\hline & $38,5 \%)$ & $(n=100)$ & $<.001$ \\
\hline
\end{tabular}




\begin{tabular}{|c|c|c|c|}
\hline$M(S D)$ & $7,43(4,04)$ & $3,7(2,46)$ & \\
\hline $\begin{array}{l}\text { Skala zu non-motorischen } \\
\text { Symptomen Frage } 28 \text { Schwere } \\
\text { keine/leicht/mittel/schwer }\end{array}$ & $\begin{array}{l}(n=115) \\
51 / 33 / 21 / 10 \\
(44,3 \% / \\
28,7 \% / \\
18,3 \% / 8,7 \%)\end{array}$ & $\begin{array}{l}(\mathrm{n}=100) \\
90 / 8 / 1 / 1 \\
(90 \% / \quad 8 \% / \\
1 \% / 1 \%)\end{array}$ & $<.001$ \\
\hline $\begin{array}{l}\text { Skala zu non-motorischen } \\
\text { Symptomen Frage } 28 \text { Häufigkeit } \\
\text { keine/selten/gelegentlich/häu- } \\
\text { fig/sehr häufig }\end{array}$ & $\begin{array}{l}(n=115) \\
51 / 2 / 3 / \quad 11 / \\
48 \\
(44,3 \% / 1,7 \% / \\
2,6 \% / \quad 9,6 \% / \\
41,7 \%)\end{array}$ & $\begin{array}{l}(n=100) \\
90 / 2 / 2 / 1 / 5 \\
(90 \% / \quad 2 \% / \\
2 \% / 1 \% / 5 \%)\end{array}$ & $<.001$ \\
\hline $\begin{array}{l}\text { Skala zu non-motorischen } \\
\text { Symptomen Frage } 28 \text { Schwere } x \\
\text { Häufigkeit } \\
M(S D)\end{array}$ & $\begin{array}{l}(n=115) \\
3,37(3,83)\end{array}$ & $\begin{array}{l}(n=100) \\
0,41(1,61)\end{array}$ & $<.001$ \\
\hline $\begin{array}{l}\text { Skala zu non-motorischen } \\
\text { Symptomen Gesamt } \\
M(S D)\end{array}$ & $\begin{array}{l}(n=114) \\
36,23(30,71)\end{array}$ & $\begin{array}{l}(n=100) \\
12,73 \\
(13,29)\end{array}$ & $<.001$ \\
\hline
\end{tabular}

$\mathrm{PD}=$ Parkinsonpatienten, $\mathrm{HC}=$ Kontrollgruppe (healthy control), $\mathrm{n}=$ Anzahl, $\mathrm{M}=$ Mittelwert, $\mathrm{SD}=\mathrm{Stan}-$ dardabweichung, PD NMS Fragebogen= Parkinson's Disease Non- Motor Symptoms Fragebogen

3.3.1.1 Persönliche Einschätzung vor der Durchführung der Riechtestungen mithilfe der Sniffin' Sticks

Zu Beginn der Testungen wurden die Patienten befragt, ob sie ihr Riechvermögen als vermindert, normal oder erhöht einschätzen würden. Hier zeigte sich ein signifikanter Unterschied zwischen der Kontrollgruppe und den Patienten ( $p<.05) .64,35$ $\%$ der Patienten gaben an, dass ihr Riechvermögen vermindert ist, wohingegen nur $13 \%$ der Kontrollpersonen dies so empfanden. $79 \%$ der Kontrollgruppe und nur 
35,65 \% der Patienten beschrieben ihren Geruchssinn als unauffällig. Es zeigte eine wesentlich größere Anzahl an Patienten eine subjektive Verschlechterung des Riechens als Probanden der Kontrollgruppe. (siehe Abb. 2)

\section{Persönliche Bewertung des Riechvermögens}

PD subject

HC- healthy control

$\square$ unauffällig/normal

$\square$ vermindert/decreased

erhöht/increased
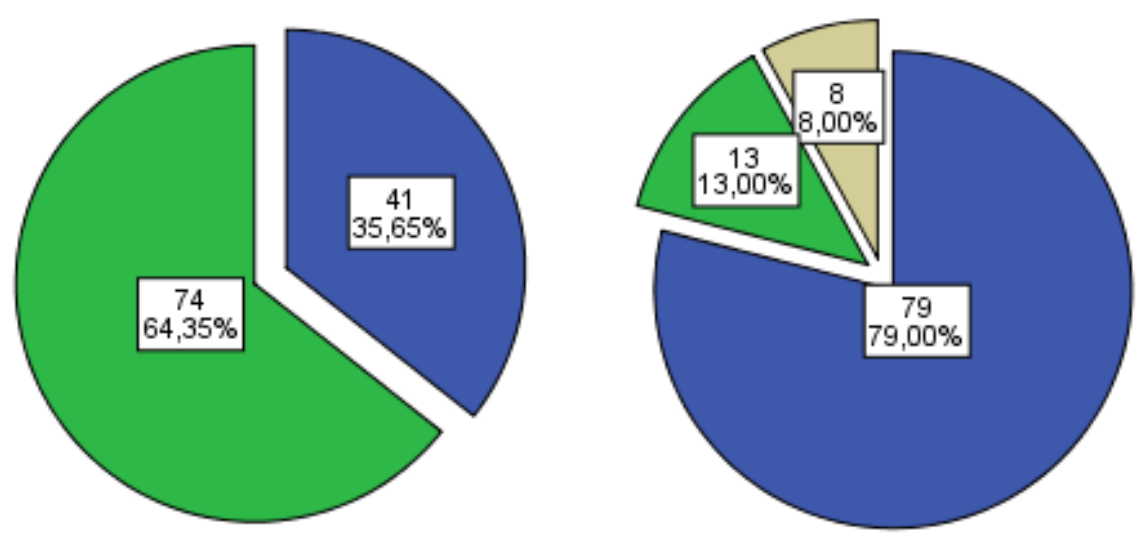

Abbildung 2 Persönliche Bewertung des Riechvermögens

\subsubsection{Fragebögen zu subjektiven Riechstörungen}

$72(61,5 \%)$ Patienten gaben in der Frage 2 des PD NMS Fragebogens bezüglich einer subjektiven Veränderungen oder einem Verlust des Riechens und Schmeckens innerhalb des letzten Monats an, eine Veränderung an, 45 (38,5\%) bemerkten keine Veränderung. Von den gesunden Probanden gaben nur 10 \% (10 Personen) eine subjektive Veränderung an, 90 \% (90 Personen) verneinten dies. Es zeigte sich ein signifikanter Unterschied $(p<.05)$. Für die Summe aller Fragen im PD NMS Fragebogen zeigte sich ebenfalls eine Signifikanz $(p<.05)$. Eine wesentlich größere Anzahl Patienten als Kontrollpersonen gab an, im Einklang mit der persönlichen Einschätzung, eine Veränderung des Riechens und Schmeckens bemerkt zu haben.

In dem Fragebogen der Skala zu non-motorischen Symptomen bei der Parkinsonerkrankung sagten 51 (43,6\%) der Patienten bei der Befragung nach der Schwere des Riechdefizits aus, dass sie keine Einschränkungen wahrgenommen haben. 33 
$(28,2 \%)$ Patienten gaben leichte Symptome an, 21 (17,9 \%) mäßige Beschwerden und $10(8,5 \%)$ eine erhebliche Belastung durch die schwere Symptomatik.

Von den insgesamt 215 zur Auswertung stehenden Antworten zu der Häufigkeit der Beschwerden, gaben 51 (43,6\%) der Patienten keine, 2 (1,7\%) seltene, 3 (2,6 \%) gelegentliche, 11 (9,4\%) häufige und 48 (41\%) sehr häufige Beeinträchtigungen durch das verminderte Riechen und Schmecken an. Bei der Kontrollgruppe gaben 90 (90\%) Personen keine, zwei (2\%) seltene, zwei (2\%) gelegentliche, eine Person (1\%) häufige und fünf (5\%) sehr häufige Beschwerden an.

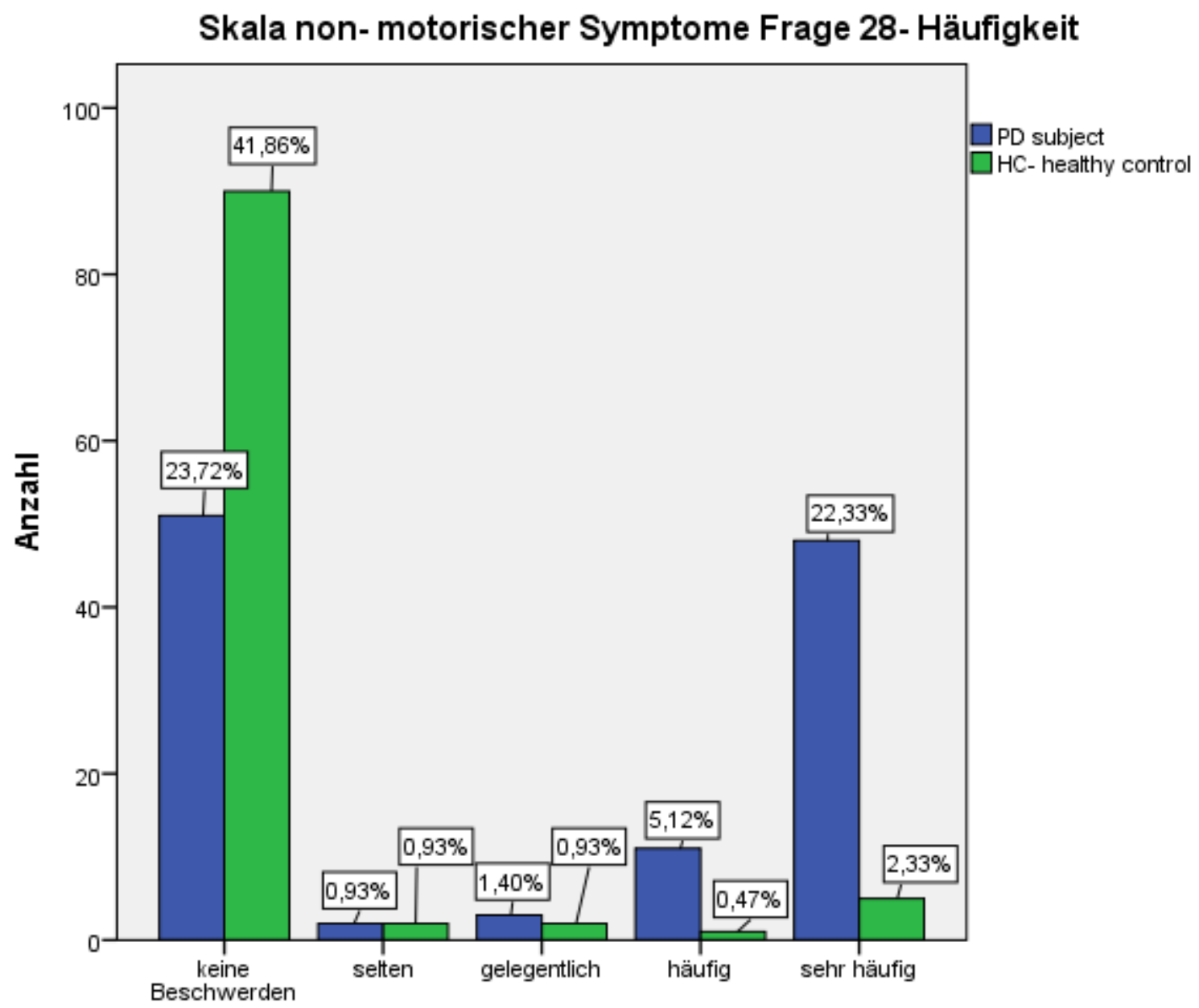

Häufigkeit der Beschwerden

Abbildung 3 Frage 28 der Skala zu non-motorischen Symptomen, Häufigkeit der Beschwerden

Bei der Berechnung des Produktes aus der Schwere und der Häufigkeit konnte ein Minimalwert von 0 Punkten und ein Maximalwert von 12 Punkten erreicht werden, im Mittel ließ sich eine Punktzahl von 3,37 Punkten für die Patienten errechnen, für die Kontrollen eine Punktzahl von 0,41. Insgesamt konnte bei allen Fragen zu der 
Skala zu non-motorischen Symptomen eine Punktzahl von 150 Punkten erreicht werden. Die Mittelwerte für die Patienten lagen bei 36,23 Punkten, die der Kontrollgruppe bei 12,73 Punkten.

Alle Auswertungen (Ausprägung, Häufigkeit, Häufigkeit x Ausprägung und die Gesamtsumme) ergaben signifikante Unterschiede zwischen der Kontrollgruppe und der Patientengruppe $(p<.05)$.

Es zeigten sich in allen Abschnitten der Testung Unterschiede zwischen den Gruppen, die Patienten gaben im Schnitt häufiger an Veränderungen beim Riechen und Schmecken bemerkt zu haben als die Kontrollgruppe.

In allen Testungen zur subjektiven Wahrnehmung des Riechens und Schmeckens unterschieden sich die verglichenen Gruppen grundlegend in ihren Angaben, eine wesentlich größere Anzahl der Patienten gab Riechstörungen an.

\subsubsection{Objektive Riechtestungen zur Schwelle, Diskrimination und} Identifikation mithilfe der Sniffin' Sticks

In den objektiven Riechtestungen zeigten sich hoch signifikante Unterschiede zwischen den getesteten Gruppen.

Tabelle 10 Objektive Riechtestungen (Schwellentest, Diskriminationstest, Identifikationstest) im Rahmen der Baseline-Datenerfassung, Durchführung mithilfe der Sniffin‘ Sticks, Signifikanz (p-Wert)

\begin{tabular}{|c|c|c|c|}
\hline & PD & $\mathrm{HC}$ & p-Wert \\
\hline Schwellentest & $(n=115)$ & $(n=100)$ & $<.001$ \\
\hline$M(S D)$ & $3,02(3,79)$ & $7,16(3,82)$ & \\
\hline \multirow{2}{*}{$\begin{array}{l}\text { Diskriminations- } \\
\text { test }\end{array}$} & $(n=115)$ & $(n=100)$ & $<.001$ \\
\hline & $8,21(3,52)$ & $11,97(2,58)$ & \\
\hline \multicolumn{4}{|l|}{$M(S D)$} \\
\hline \multirow{3}{*}{$\begin{array}{l}\text { Identifikations- } \\
\text { test Gesamt } \\
\text { M (SD) }\end{array}$} & $(\mathrm{n}=117)$ & $(n=100)$ & $<.001$ \\
\hline & $6,91(3,36)$ & $11,91(2,55)$ & \\
\hline & & & \\
\hline
\end{tabular}


Im Schwellen-, Diskriminations- und Identifikationstest erzielten die Testpersonen in der Kontrollgruppe signifikant höhere Punktzahlen als in der Gruppe der Parkinsonpatienten. Dieses Ergebnis weist auf eine deutlich bessere Riechfunktion hin.

\subsubsection{Schwellentest}

Der Schwellentest zeigte für die verglichenen Gruppen, Patienten- und Kontrollgruppe, signifikante Unterschiede $(p<.05)$. Im Schwellentest erzielten die Patienten einen Mittelwert von 3,04 Punkten, die Kontrollpersonen eine Punktzahl von 7,16.

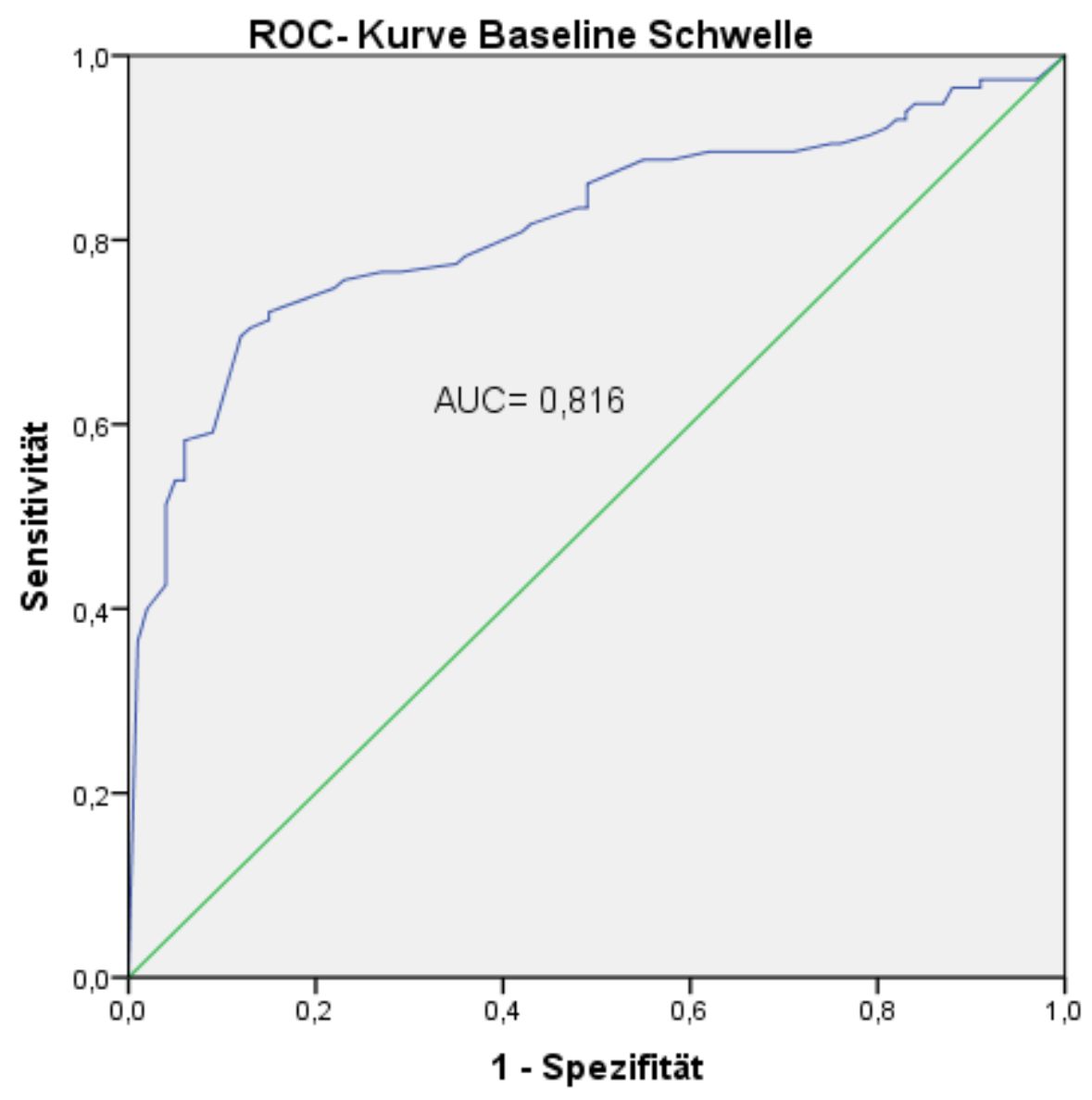

Abbildung 4 ROC-Kurve Schwellentest

In der ROC-Kurve zeigt sich eine Fläche unter der Kurve (AUC) von 0,816, was für eine gute Testgüte spricht. 


\subsubsection{Diskriminationstest}

Bei einem $p$-Wert von $p<.001$ ließ sich ebenfalls ein signifikanter Unterschied im Diskriminationstest nachweisen. Im Mittel erreichten die Patienten eine Punktzahl von 8,23, die Kontrollen 12,02 Punkte.

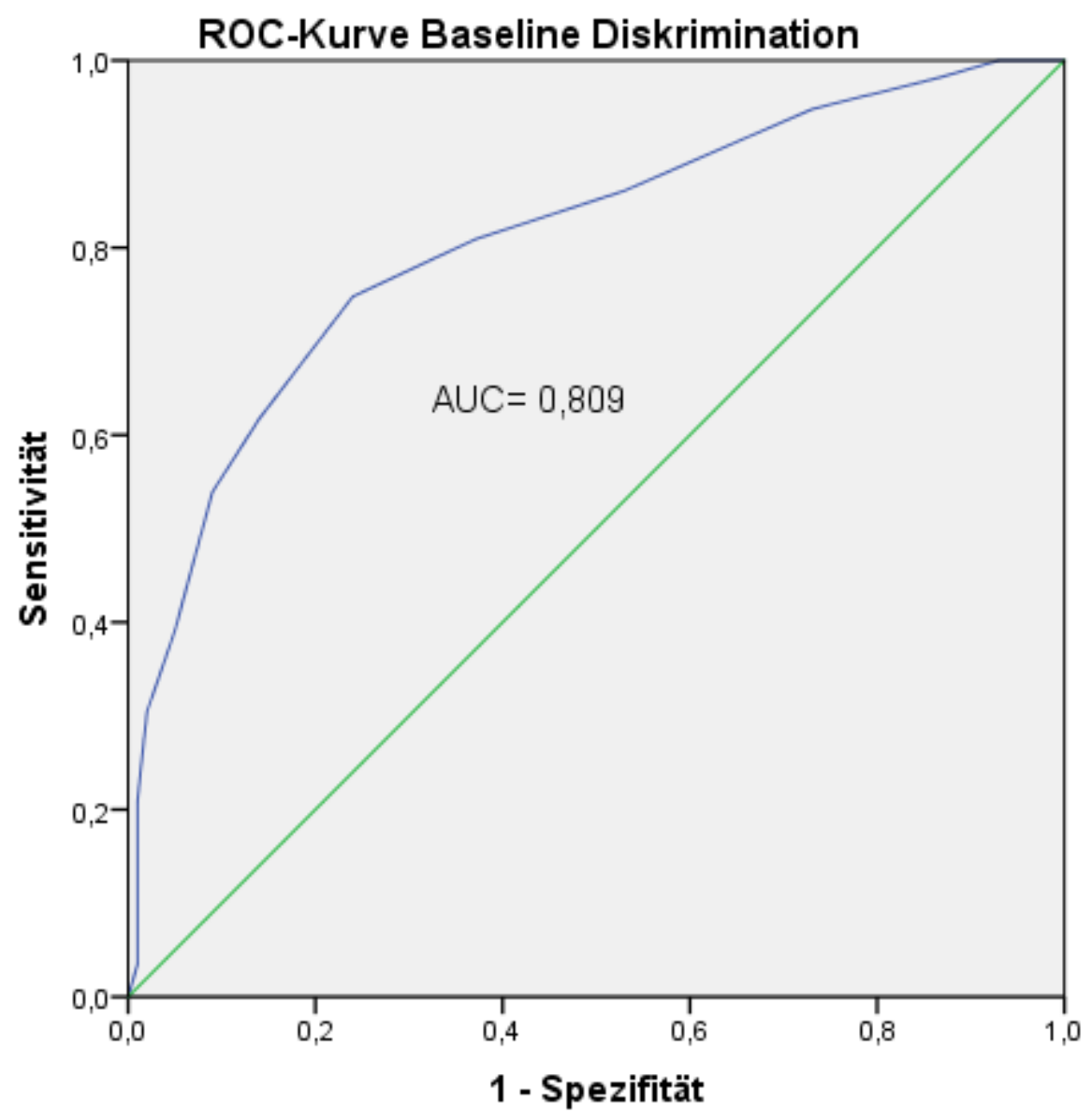

Abbildung 5 ROC-Kurve Diskriminationstest

Die AUC in der ROC-Kurve beträgt 0,809. 


\subsubsection{Identifikationstest Gesamt}

Im Identifikationstest Gesamt wurden alle korrekt benannten Qualitäten der $16 \mathrm{Te}$ stungen gezählt. Auch hier lag der $p$-Wert bei $p<.001$. Der Mittelwert lag bei den Patienten bei 6,87 Punkten, der der Kontrollgruppe bei 11,96.

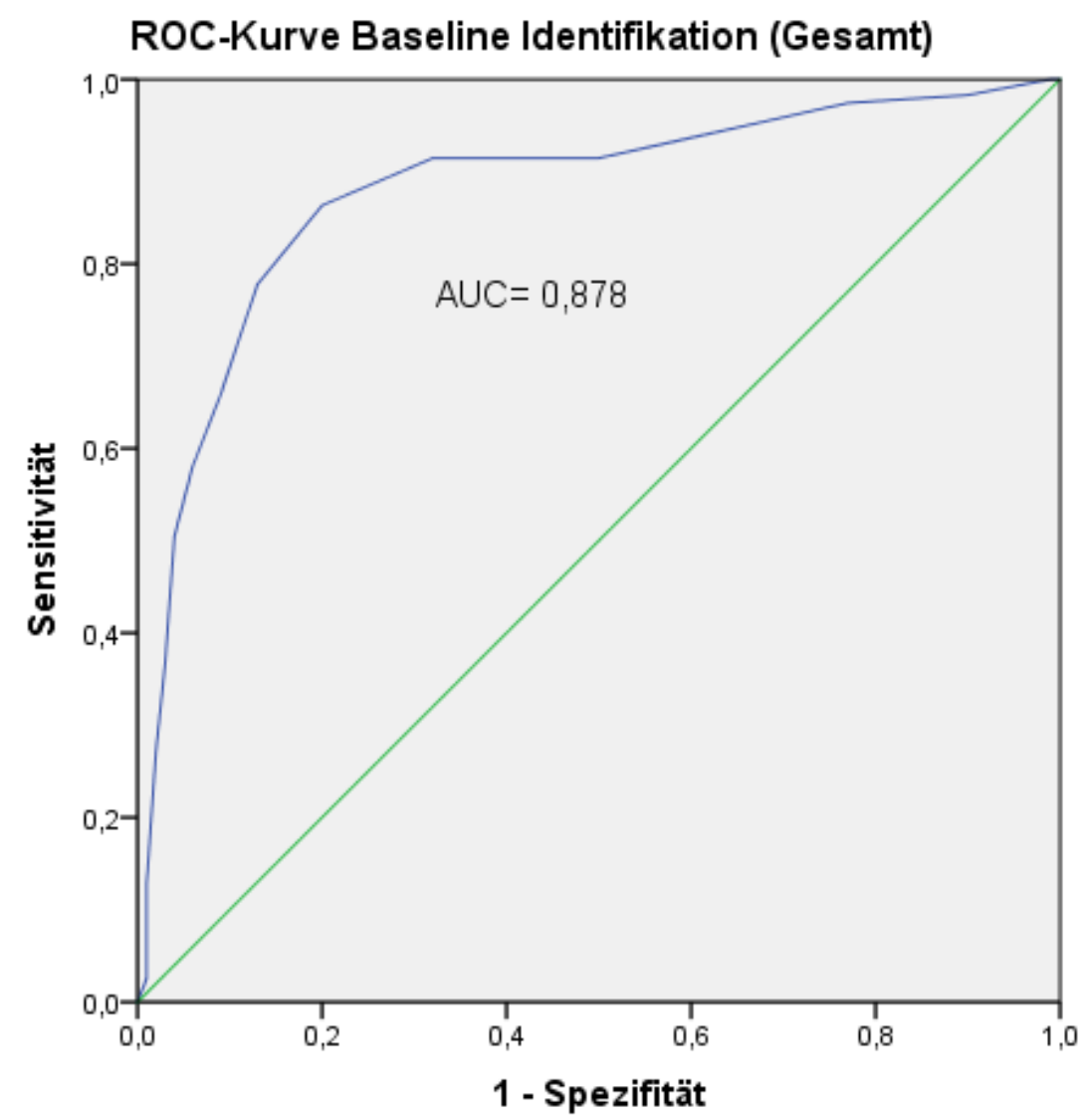

Abbildung 6 ROC-Kurve Identifikationstest

Die Area Under The Curve in der ROC-Kurve beträgt 0,878.

Im Schwellentest erzielten die Patienten im Mittel geringere Punktzahlen als die Kontrollpersonen. Auch im Diskriminationstest und im Identifikationstest Gesamt zeigte sich dies. Die Testergebnisse deuten im Vergleich zu der Kontrollgruppe auf einen abgeschwächten Geruchssinn bei den Patienten hin.

Für alle Tests zeigte sich in der ROC-Kurve eine AUC $>0,8$ und folglich eine gute Testgüte. 


\subsubsection{Identifikationstest 1-16}

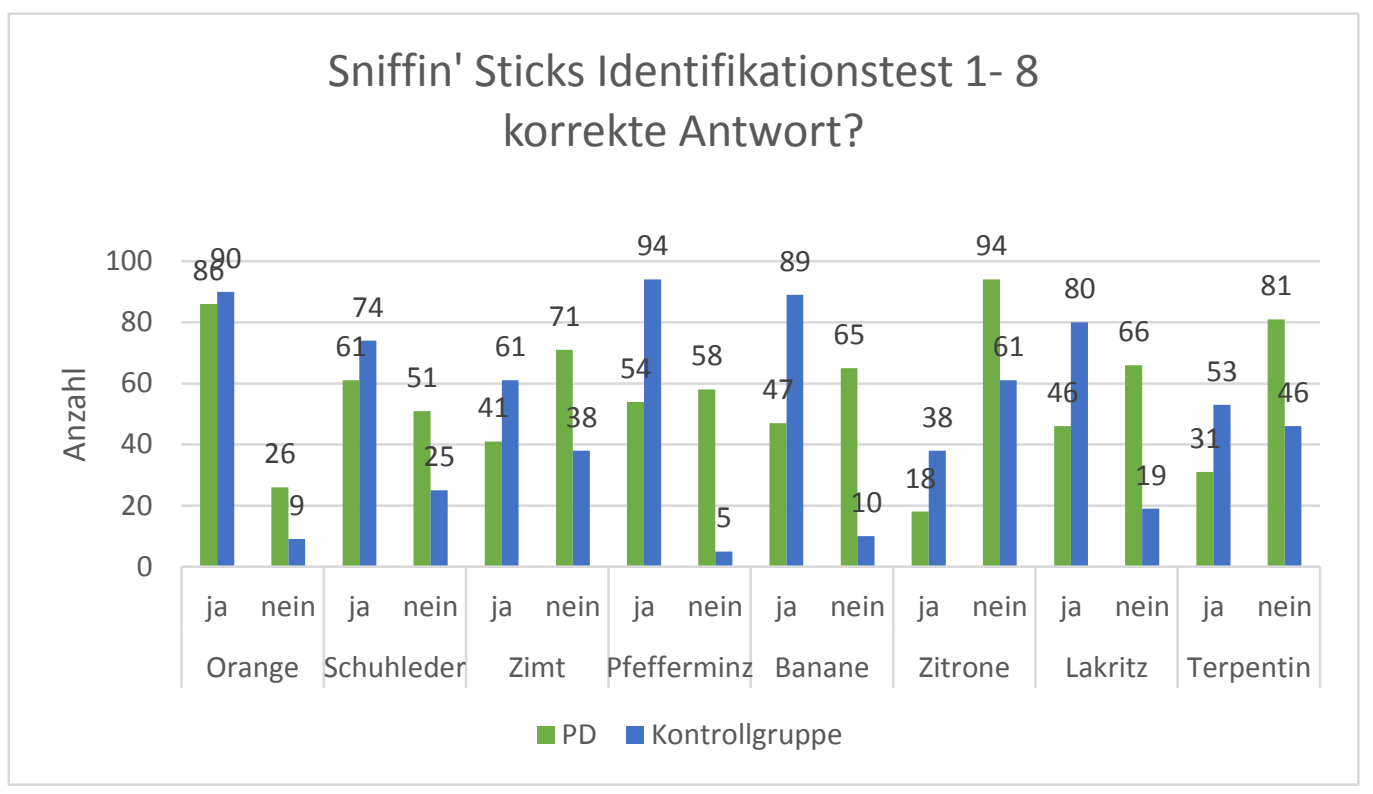

Abbildung 7 Korrekte Antworten im Sniffin‘ Sticks-Identifikationstest 1-8

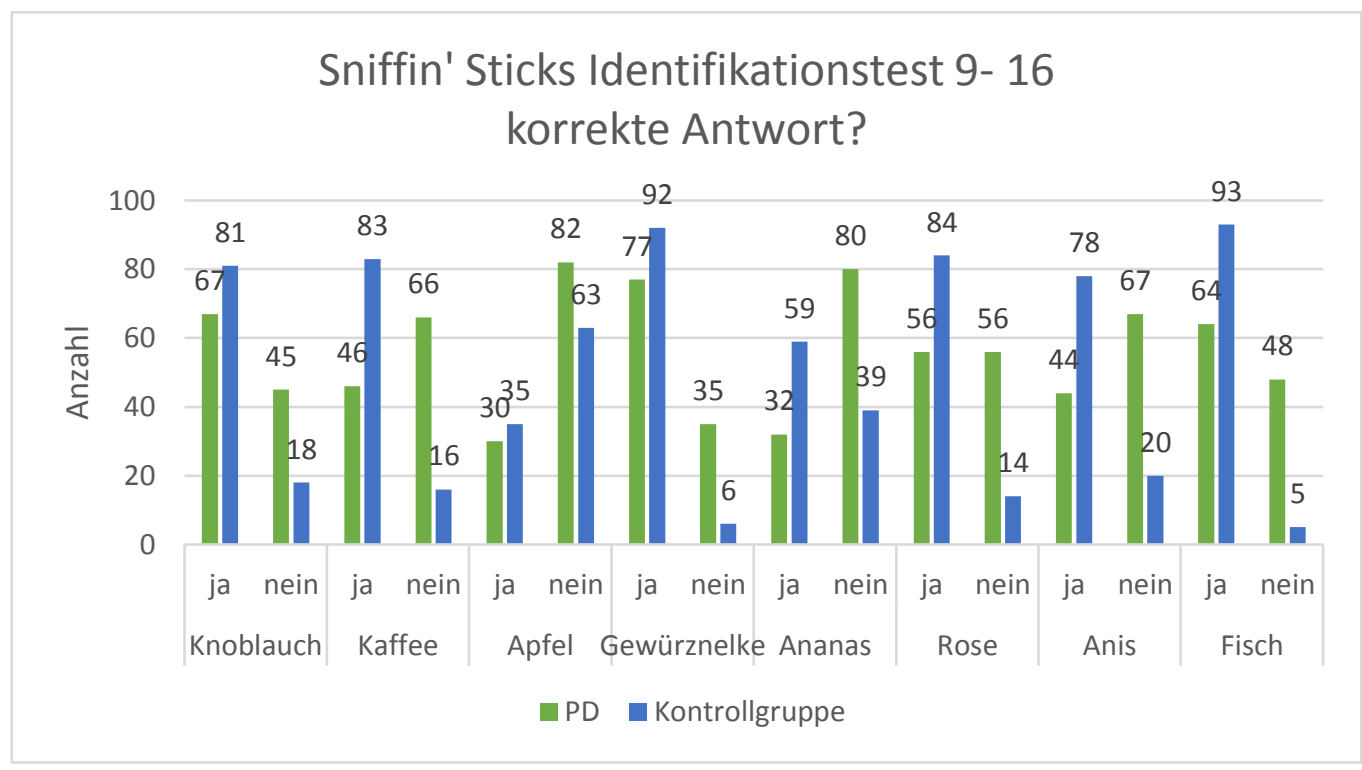

Abbildung 8 Korrekte Antworten im Sniffin‘ Sticks-Identifikationstest 9-16

Die meisten Falschantworten der Patienten (94 Falschantworten bei 18 korrekten Antworten) zeigten sich im Test 6, bei dem der Duftstoff „Zitrone“ erkannt werden sollte. Die zweithäufigste Anzahl an Falschantworten zeigte Test 11, in dem der Geruch „Apfel“ genannt werden sollte. Hier beantworteten 73,2 \% (82 Probanden) der Patienten die Frage falsch, 26,8 \% (30 Probanden) gaben die korrekte Antwort. 
Test 8 (Terpentin) schnitt mit 81 Falschantworten und 31 korrekten Antworten am drittschlechtesten ab.

Die besten Ergebnisse erzielten die Patienten bei dem Test 1, wo „Orange“ erkannt werden musste. Hierbei gaben 86 Patienten die korrekte Antwort an, 26 die falsche Antwort. Die zweitbesten Testergebnisse zeigte Test 12 (Gewürznelke) mit 77 korrekten Antworten und 35 falschen Antworten. Den Geruch „Knoblauch“ (Test 9) erkannten 67 Patienten (45 Falschantworten), womit der Test 9 am drittstärksten abschnitt.

Zwischen den Patienten und der Kontrollgruppe zeigten sich in allen Testungen, außer im Test Nr.11- Apfel ( $p=.163)$, signifikante Unterschiede.

Tabelle 11 Patienten Identifikationstests 1-16, Häufigkeitstabelle und p-Werte, Anzahl (\%)

\begin{tabular}{|c|c|c|c|c|c|c|}
\hline \multirow{2}{*}{$\begin{array}{l}\text { Sniffin' Sticks- } \\
\text { Identifikationstest }\end{array}$} & \multicolumn{2}{|c|}{ PD } & \multicolumn{2}{|c|}{ KG } & \multirow{2}{*}{$\begin{array}{l}\text { Feh- } \\
\text { lende } \\
\text { Antwor- } \\
\text { ten }\end{array}$} & \multirow{2}{*}{$\begin{array}{l}\text { p- } \\
\text { Wert }\end{array}$} \\
\hline & $\begin{array}{l}\text { korrekte } \\
\text { Antwort }\end{array}$ & $\begin{array}{l}\text { falsche } \\
\text { Antwort }\end{array}$ & $\begin{array}{l}\text { korrekte } \\
\text { Antwort }\end{array}$ & $\begin{array}{l}\text { falsche } \\
\text { Antwort }\end{array}$ & & \\
\hline Orange (1) & $\begin{array}{c}86 \\
(76,8)\end{array}$ & $\begin{array}{c}26 \\
(23,2)\end{array}$ & $\begin{array}{c}90 \\
(90,9)\end{array}$ & $9(9,1)$ & 6 & .006 \\
\hline Schuhleder (2) & $\begin{array}{c}61 \\
(54,5)\end{array}$ & $\begin{array}{c}51 \\
(45,5)\end{array}$ & $\begin{array}{c}74 \\
(74,7)\end{array}$ & $\begin{array}{c}25 \\
(25,3)\end{array}$ & 6 & .002 \\
\hline Zimt (3) & $\begin{array}{c}41 \\
(36,6)\end{array}$ & $\begin{array}{c}71 \\
(63,4)\end{array}$ & $\begin{array}{c}61 \\
(61,6)\end{array}$ & $\begin{array}{c}38 \\
(38,4)\end{array}$ & 6 & $<.001$ \\
\hline Pfefferminz (4) & $\begin{array}{c}54 \\
(48,2)\end{array}$ & $\begin{array}{c}58 \\
(51,8)\end{array}$ & $\begin{array}{c}94 \\
(94,9)\end{array}$ & $5(5,1)$ & 6 & $<.001$ \\
\hline Banane (5) & $47(42)$ & $65(58)$ & $\begin{array}{c}89 \\
(89,9)\end{array}$ & $\begin{array}{c}10 \\
(10,1)\end{array}$ & 6 & $<.001$ \\
\hline Zitrone (6) & $\begin{array}{c}18 \\
(16,1)\end{array}$ & $\begin{array}{c}94 \\
(83,9)\end{array}$ & $\begin{array}{c}38 \\
(38,4)\end{array}$ & $\begin{array}{c}61 \\
(61,6)\end{array}$ & 6 & $<.001$ \\
\hline Lakritz (7) & $\begin{array}{c}46 \\
(41,4)\end{array}$ & $\begin{array}{c}66 \\
(58,9)\end{array}$ & $\begin{array}{c}80 \\
(80,8)\end{array}$ & $\begin{array}{c}19 \\
(19,2)\end{array}$ & 6 & $<.001$ \\
\hline
\end{tabular}




\begin{tabular}{|c|c|c|c|c|c|c|}
\hline Terpentin (8) & $\begin{array}{c}31 \\
(27,7)\end{array}$ & $\begin{array}{c}81 \\
(72,3)\end{array}$ & $\begin{array}{c}53 \\
(53,5)\end{array}$ & $\begin{array}{c}46 \\
(46,5)\end{array}$ & 6 & $<.001$ \\
\hline Knoblauch (9) & $\begin{array}{c}67 \\
(59,8)\end{array}$ & $\begin{array}{c}45 \\
(40,2)\end{array}$ & $\begin{array}{c}81 \\
(81,8)\end{array}$ & $\begin{array}{c}18 \\
(18,2)\end{array}$ & 6 & $<.001$ \\
\hline Kaffee (10) & $\begin{array}{c}46 \\
(41,1)\end{array}$ & $\begin{array}{c}66 \\
(58,9)\end{array}$ & $\begin{array}{c}83 \\
(83,8)\end{array}$ & $\begin{array}{c}16 \\
(16,2)\end{array}$ & 6 & $<.001$ \\
\hline Apfel (11) & $\begin{array}{c}30 \\
(26,8)\end{array}$ & $\begin{array}{c}82 \\
(73,2)\end{array}$ & $\begin{array}{c}35 \\
(35,7)\end{array}$ & $\begin{array}{c}63 \\
(64,3)\end{array}$ & 7 & .163 \\
\hline Gewürznelke (12) & $\begin{array}{c}77 \\
(68,8)\end{array}$ & $\begin{array}{c}35 \\
(31,3)\end{array}$ & $\begin{array}{c}92 \\
(93,9)\end{array}$ & $6(6,1)$ & 7 & $<.001$ \\
\hline Ananas (13) & $\begin{array}{c}32 \\
(28,6)\end{array}$ & $\begin{array}{c}80 \\
(71,4)\end{array}$ & $\begin{array}{c}59 \\
(60,2)\end{array}$ & $\begin{array}{c}39 \\
(39,8)\end{array}$ & 7 & $<.001$ \\
\hline Rose (14) & $56(50)$ & $56(50)$ & $\begin{array}{c}84 \\
(85,7)\end{array}$ & $\begin{array}{c}14 \\
(14,3)\end{array}$ & 7 & $<.001$ \\
\hline Anis (15) & $\begin{array}{c}44 \\
(39,6)\end{array}$ & $\begin{array}{c}67 \\
(60,4)\end{array}$ & $\begin{array}{c}78 \\
(79,6)\end{array}$ & $\begin{array}{c}20 \\
(20,4)\end{array}$ & 8 & $<.001$ \\
\hline Fisch (16) & $\begin{array}{c}64 \\
(57,1)\end{array}$ & $\begin{array}{c}48 \\
(42,9)\end{array}$ & $\begin{array}{c}93 \\
(94,9)\end{array}$ & $5(5,1)$ & 7 & $<.001$ \\
\hline
\end{tabular}

\subsubsection{TDI-Score}

Eine Punktzahl $\geq 30$ im TDI-Score spricht für eine Normosmie, 16-29 Punkte für eine Hyposmie und $\leq 15$ Punkte für eine Anosmie. Bei den Patienten wurden 115 Antworten gewertet, bei der Kontrollgruppe 100. Die Patienten erreichten im TDIScore einen signifikant niedrigeren Mittelwert (18,22 Punkte) als die Kontrollgruppe (31,04 Punkte) und liegen damit deutlich im Bereich der Hyposmie. (s. Abb. 9)

Zwei Kontrollpersonen erzielten Ergebnisse in den Testungen, die für eine Anosmie sprechen, während bei den Patienten 47 Probanden als anosmisch bezeichnet werden können. Eine Hyposmie wiesen 56 Patienten und 39 Kontrollpersonen 
auf. Einen normalen Geruchssinn zeigten laut TDI-Score 12 Patienten und 59 Kontrollpersonen. Es zeigt sich ein hoch signifikanter Unterschied von $p<.001$.

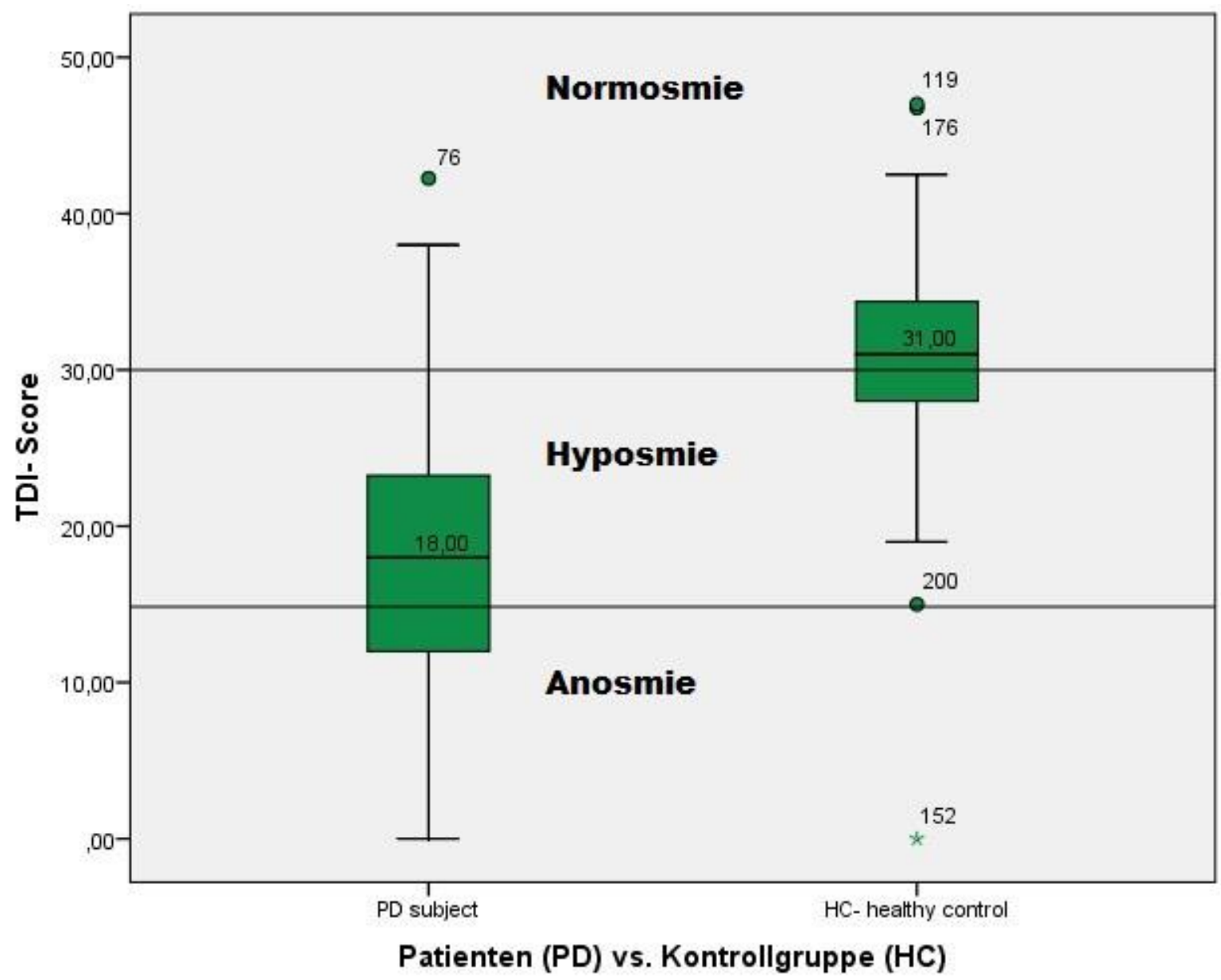

Abbildung 9 Box Plots TDI-Score

\subsubsection{Sensitivität, Spezifität und Youden-Index des Riechidentifikationstests}

Die höchste Sensitivität zeigte sich bei der Qualität „Zitrone“ mit 83,93 \%. Die zweithöchste Sensitivität zeigte die Qualität „Apfel“ mit 73,21 \%, am drittstärksten erwies sich der Geruch „Terpentin“ mit 72,32\%.

Bezogen auf die Spezifität lag der Höchstwert bei 94,95 \% mit dem Geruch „Pfefferminz", darauf folgend zeigte sich die Qualität "Gewürznelke“ mit 93,88 \% und "Orange“ mit 90,91\%.

Der Youden-Index wird berechnet durch die Sensitivität + Spezifität - 1. Der höchste Youden-Index zeigte die Qualität „Banane“ mit 0,4794, der zweithöchste Werte 
wurde für die Qualität „Pfefferminz“ errechnet $(0,4674)$. Am drittstärksten schnitt der Geruchsstoff „Kaffee“ mit einem Youden-Index von 0,4277 ab.

Tabelle 12 Sensitivität, Spezifität und Youden-Index (= Sensitivität+ Spezifität- 1) des Identifikationstests 1-16 der Patienten

\begin{tabular}{|c|c|c|c|}
\hline Identifikationstest & Sensitivität & Spezifität & Youden Index \\
\hline Orange (1) & $23,21 \%$ & $90,91 \%$ & 0,1412 \\
\hline Schuhleder (2) & $45,54 \%$ & $74,75 \%$ & 0,2029 \\
\hline Zimt (3) & $63,39 \%$ & $61,62 \%$ & 0,2501 \\
\hline Pfefferminz (4) & $51,79 \%$ & $94,95 \%$ & 0,4674 \\
\hline Banane (5) & $58,04 \%$ & $89,90 \%$ & 0,4794 \\
\hline Zitrone (6) & $83,93 \%$ & $38,38 \%$ & 0,2231 \\
\hline Lakritz (7) & $58,93 \%$ & $80,81 \%$ & 0,3974 \\
\hline Terpentin (8) & $72,32 \%$ & $53,54 \%$ & 0,2586 \\
\hline Knoblauch (9) & $40,18 \%$ & $81,82 \%$ & 0,22 \\
\hline Kaffee (10) & $58,93 \%$ & $83,84 \%$ & 0,4277 \\
\hline Apfel (11) & $73,21 \%$ & $35,71 \%$ & 0,0892 \\
\hline Gewürznelke (12) & $31,25 \%$ & $93,88 \%$ & 0,2513 \\
\hline Ananas (13) & $71,43 \%$ & $60,20 \%$ & 0,3163 \\
\hline Rose (14) & $50,00 \%$ & $85,71 \%$ & 0,3571 \\
\hline Anis (15) & $60,36 \%$ & $79,59 \%$ & 0,3995 \\
\hline Fisch (16) & $42,86 \%$ & $94,90 \%$ & 0,3776 \\
\hline
\end{tabular}




\subsection{Korrelationen der Baseline-Daten}

3.4.1 Korrelationen Riechtest Schwelle, Diskrimination und Identifikation Gesamt In den nachfolgenden Tabellen werden die objektiven Riechtestungen, die mit Hilfe der Sniffin' Sticks durchgeführt wurden, mit weiteren Testungen korreliert.

Tabelle 3 beschreibt die Korrelationen der Patienten. Die Werte, die eine Korrelation beschreiben, sind dabei hervorgehoben.

Tabelle 3 Korrelationen der Patienten, Korrelationskoeffizient (r) der Sniffin' Sticks Schwelle, Diskrimination, Identifikation Gesamt und p-Wert (in Klammern)

\begin{tabular}{|c|c|c|c|}
\hline & \multicolumn{3}{|c|}{ Sniffin' Sticks } \\
\hline & $\begin{array}{l}\text { Schwellen- } \\
\text { test }\end{array}$ & Diskriminationstest & $\begin{array}{l}\text { Identifikationstest } \\
\text { Gesamt }\end{array}$ \\
\hline Alter & $\begin{array}{c}-0,345(< \\
.05)\end{array}$ & $-0,224(<.05)$ & $-\mathbf{0 , 2 0 0}(.05)$ \\
\hline $\begin{array}{l}\text { Erkrankungs- } \\
\text { dauer }\end{array}$ & $\begin{array}{c}0,037( \\
.696)\end{array}$ & $\mathbf{0 , 1 1 7}(.214)$ & 0,097 (.298) \\
\hline
\end{tabular}

\begin{tabular}{l|l|l|l|}
$\begin{array}{l}\text { Persönliche Ein- } \\
\text { schätzung }\end{array}$ & $-0,472(<.05)$ & $-0,429(<.05)$ & $-0,427(<.05)$ \\
\hline $\begin{array}{l}\text { Parkinson's Dise- } \\
\text { ase Fragebogen } \\
\text { Gesamt }\end{array}$ & $-0,200(<.05)$ & $-0,195(<.05)$ & $-0,119(.202)$ \\
\hline $\begin{array}{l}\text { Skala zu non-mo- } \\
\text { torischen Symp- } \\
\text { tomen Frage 28 } \\
\text { Schwere }\end{array}$ & $-0,560(<.05)$ & $-0,450(<.05)$ & $-0,365(<.05)$ \\
\hline
\end{tabular}




\begin{tabular}{|c|c|c|c|}
\hline $\begin{array}{l}\text { Skala zu non-mo- } \\
\text { torischen Symp- } \\
\text { tomen Frage } 28 \\
\text { Häufigkeit }\end{array}$ & $-0,520(<.05)$ & $-0,382(<.05)$ & $-0,331(<.05)$ \\
\hline $\begin{array}{l}\text { Skala zu non-mo- } \\
\text { torischen Symp- } \\
\text { tomen Frage } 28 \\
\text { HxS }\end{array}$ & $-0,561(<.05)$ & $-0,461(<.05)$ & $-0,359(<.05)$ \\
\hline $\begin{array}{l}\text { Skala zu non-mo- } \\
\text { torischen Symp- } \\
\text { tomen Frage } 28 \\
\text { Gesamt }\end{array}$ & $-0,147(.121)$ & $-0,181(.054)$ & $-0,077(.418)$ \\
\hline Uhrentest & $-0,073(.436)$ & $-0,162(.084)$ & $-0,076(.419)$ \\
\hline MMST & $0,017(.858)$ & $\mathbf{0 , 1 4 1}(.134)$ & $\mathbf{0 , 1 5 4}(.099)$ \\
\hline GDS & $-0,040(.675)$ & $-0,046(.633)$ & 0,039 (.682) \\
\hline$B D I$ & $-0,047(.624)$ & $-0,081(.396)$ & $0,060(.525)$ \\
\hline MADRS & 0,009 (.926) & $-0,056(.558)$ & $-0,055(.568)$ \\
\hline MDS UPDRS I & $-0,059$ (.533) & $-0,095$ (.312) & 0,003 (.971) \\
\hline MDS UPDRS II & $-0,091(.336)$ & - 0,169 (.070) & $-0,089(.342)$ \\
\hline MDS UPDRS III & $-0,077(.415)$ & - 0,218 (.019) & $-0,138(.137)$ \\
\hline $\begin{array}{l}\text { MDS UPDRS Ge- } \\
\text { samt }\end{array}$ & $-0,095(.314)$ & $-0,218(.019)$ & $-\mathbf{0 , 1 2 1}(.195)$ \\
\hline
\end{tabular}

Es zeigten sich sowohl negative als auch positive Korrelationen. 
Bezogen auf das Alter der Patienten zeigten sich gering- bis mittelgradige negative Korrelationen. Demzufolge erzielten die Probanden geringere Testwerte in den Riechtests, je älter die Patienten waren.

Die Erkrankungsdauer korrelierte geringgradig positiv mit dem Diskriminationstest. In Bezug auf die Abschnitte der Frage 28 der Skala zu non-motorischen Symptomen und dem PD NMS Fragebogen präsentierten sich ausschließlich geringgradige bis hochgradige, negative Korrelationen. Demzufolge geht ein hoher Score bei der Frage 28 zu der Schwere, Häufigkeit, HxS und Gesamt der Skala zu non-motorischen Symptomen und der PD NMS Gesamt Testung mit einem geringen Wert bei den Riechtests einher. Wenn subjektiv eine Veränderung des Riechens wahrgenommen wurde, ließ sich das in den objektiven Riechtests zur Schwelle, Diskrimination und Identifikation Gesamt auch nachvollziehen.

Für den Uhrentest stellte sich mit dem Diskriminationstest eine geringgradige negative Korrelation dar. Erzielten die Patienten im Uhrentest hohe Testwerte, die für eine kognitive Einschränkung sprechen, fielen auch die Auswertungen des Diskriminationstests schlecht aus. Eine geringgradige positive Korrelation zeigte sich bei dem Mini-Mental-State Test (MMST) mit dem Diskriminations- und dem Identifikationstest. Je besser die kognitive Leistung im MMST war, umso besser war auch das Riechvermögen der Patienten.

Die Depressionstestungen korrelierten nicht mit den Riechtestungen.

Die Spearman-Korrelation zeigte für die MDS UPDRS Testungen und vor allem für den Diskriminationstest geringgradige, negative, lineare Korrelationen. Dies deutet daraufhin, dass hohe Scores in den UPDRS Testungen und damit eine vorliegende pathognomonische Symptomatik des idiopathischen Parkinsonsyndroms mit geringen Scores bei den Riechtests und damit einem verminderten Riechvermögen einhergehen.

In Tabelle 5 werden die Korrelationen der Kontrollgruppe beschrieben. 
Tabelle 5 Korrelationen der Kontrollgruppe, Korrelationskoeffizient (r) der Riechtestungen Schwelle, Diskrimination und Identifikation Gesamt, p-Wert (in Klammern)

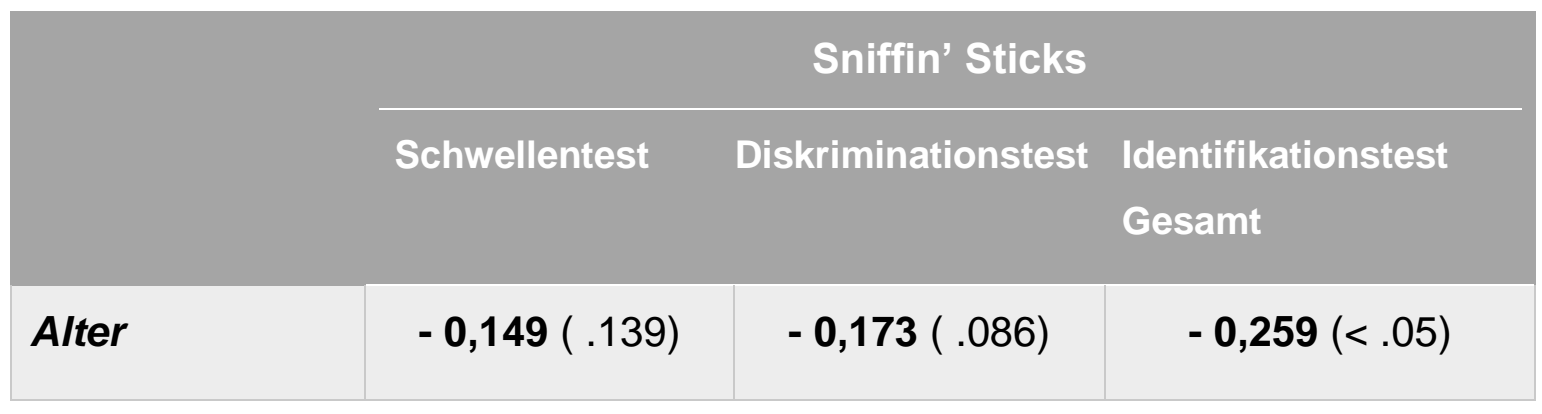

\begin{tabular}{|c|c|c|c|}
\hline $\begin{array}{l}\text { Persönliche Ein- } \\
\text { schätzung }\end{array}$ & $-\mathbf{0 , 2 6 8}(<.05)$ & 0,007 ( .944) & $0,002(.982)$ \\
\hline PD NMS Gesamt & $-\mathbf{0 , 1 2 9}(.199)$ & $-0,011(.916)$ & $-0,022(.826)$ \\
\hline $\begin{array}{l}\text { Skala zu non- } \\
\text { motorischen } \\
\text { Symptomen } \\
\text { Frage } 28 \\
\text { Schwere }\end{array}$ & $-0,116(.251)$ & $-0,180(.073)$ & $-0,049(.630)$ \\
\hline $\begin{array}{l}\text { Skala zu non- } \\
\text { motorischen } \\
\text { Symptomen } \\
\text { Frage } 28 \text { Häufig- } \\
\text { keit }\end{array}$ & $-0,118(.241)$ & $-0,174(.084)$ & $-0,050(.621)$ \\
\hline $\begin{array}{l}\text { Skala zu non- } \\
\text { motorischen } \\
\text { Symptomen } \\
\text { Frage } 28 \mathrm{H} \text { x S }\end{array}$ & $-0,120(.233)$ & $-0,178(.077)$ & $-0,053(.597)$ \\
\hline $\begin{array}{l}\text { Skala zu non- } \\
\text { motorischen } \\
\text { Symptomen }\end{array}$ & $-0,029(.772)$ & $0,036(.720)$ & $-0,043(.668)$ \\
\hline
\end{tabular}




\section{Frage 28 Ge- samt}

\begin{tabular}{|l|l|l|l|}
\hline Uhrentest & $-\mathbf{0 , 1 1 6}(.253)$ & $-0,076(.456)$ & $-\mathbf{0 , 1 2 9}(.202)$ \\
\hline MMST & $-0,003(.976)$ & $-0,006(.956)$ & $\mathbf{0 , 1 6 8}(.101)$ \\
\hline
\end{tabular}

\begin{tabular}{|l|l|l|l|}
\hline GDS & $-0,080(.434)$ & $0,015(.881)$ & $-\mathbf{0 , 1 6 9}(.096)$ \\
\hline BDI & $-0,034(.741)$ & $-0,007(.946)$ & $-\mathbf{0 , 1 4 2}(.160)$ \\
\hline MADRS & $-\mathbf{0 , 1 1 1}(.273)$ & $0,018(.856)$ & $-0,059(.563)$ \\
\hline
\end{tabular}

\begin{tabular}{|l|c|c|c|}
\hline MDS UPDRS I & $-0,025(.807)$ & $0,086(.396)$ & $-0,010(.924)$ \\
\hline MDS UPDRS II & $-0,001(.990)$ & $-0,056(.577)$ & $\mathbf{0 , 1 1 0}(.277)$ \\
\hline MDS UPDRS III & $-0,095(.349)$ & $-\mathbf{0 , 1 5 3}(.129)$ & $-\mathbf{0 , 1 6 5}(.100)$ \\
\hline MDS UPDRS Ge- & $-0,042(.678)$ & $-0,035(.727)$ & $-0,059(.560)$ \\
\hline samt & & & \\
\hline
\end{tabular}

Insgesamt korrelierten nur einige Testungen der Kontrollgruppe geringgradig miteinander.

Das Alter der Kontrollgruppe korrelierte geringgradig negativ mit den Riechtests.

Für die subjektiven Riechtestungen zeigten sich des Weiteren negative Korrelationen, vor allem bezogen auf die Schwellen- und Diskriminationstests.

Der Uhrentest korrelierte negativ mit dem Diskriminationstest und dem Identifikationstest, während der Mini-Mental State Test im Vergleich eine positive Korrelation mit dem Identifikationstest zeigte.

Der Schwellen- und Identifikationstest korrelierte teilweise geringgradig negativ mit den Depressionstestungen GDS, BDI und MADRS. Es gingen demzufolge hohe 
Werte in den Depressionstests und damit eine Tendenz zur Depression mit niedrigen Scores bei den Riechtests und damit einem herabgesetzten Geruchssinn einher.

Außerdem stellten sich für die MDS UPRDS Klassifikation geringe negative Korrelationen im Diskriminations- und Identifikationstest dar.

\subsection{Folgeuntersuchung der Riechtestungen nach 24 Monaten}

Im Rahmen der Folgeuntersuchung nach 24 Monaten wurden erneut umfangreiche Testungen durchgeführt. Nachfolgend werden diese im Vergleich zu der Baseline Testung dargestellt.

\subsubsection{Subjektive Riechtestungen}

Tabelle 13 Subjektive Riechtestungen, Vergleich Baseline- und Folgeuntersuchung nach 24 Monaten, p-Wert für die Patienten in der Baseline (BL)- und Folgeuntersuchung (FU)

\begin{tabular}{|c|c|c|c|c|c|}
\hline \multirow[t]{2}{*}{ Tests } & \multicolumn{2}{|c|}{ PD } & \multicolumn{2}{|c|}{ KG } & \multirow{2}{*}{$\begin{array}{l}\text { p-Wert } \\
\text { PD BL vs. FU }\end{array}$} \\
\hline & BL & FU & BL & FU & \\
\hline $\begin{array}{l}\text { Persönliche Be- } \\
\text { wertung } \\
\text { normal/vermin- } \\
\text { dert/erhöht } \\
\text { (\%) }\end{array}$ & $\begin{array}{l}(n=115) \\
41 / 74 / 0 \\
(35,65 / \\
64,35 / 0)\end{array}$ & $\begin{array}{c}(n=114) \\
15 / 98 / 1 \\
(13,2 / \\
86 / 0,9)\end{array}$ & $\begin{array}{l}(n=100) \\
79 / 13 / 8 \\
(79 / 13 / 1 \\
8)\end{array}$ & $\begin{array}{c}(n=95) \\
71 / 24 / 0 \\
(74,7 / \\
25,3 / 0)\end{array}$ & $<.001$ \\
\hline $\begin{array}{l}\text { PD NMS Fragebo- } \\
\text { gen Frage } 2 \\
\text { ja/nein (\%) }\end{array}$ & $\begin{array}{l}(n=117) \\
72(61,5) / \\
45(38,5)\end{array}$ & $\begin{array}{l}(\mathrm{n}=117) \\
11 \quad(9,4) / \\
106 \\
(90,6)\end{array}$ & $\begin{array}{l}(\mathrm{n}=100) \\
10 \quad(10) / \\
90(90)\end{array}$ & $\begin{array}{l}(n=100) \\
3(3) / 97 \\
(97)\end{array}$ & $<.001$ \\
\hline $\begin{array}{l}\text { PD NMS Fragebo- } \\
\text { gen Gesamt } \\
M(S D)\end{array}$ & $\begin{array}{l}(n=117) \\
7,43 \\
(4,04)\end{array}$ & $\begin{array}{l}(n=117) \\
8,43 \\
(4,28)\end{array}$ & $\begin{array}{l}(n=100) \\
3,7(2,46)\end{array}$ & $\begin{array}{l}(n=100) \\
4,12 \\
(2,92)\end{array}$ & .003 \\
\hline
\end{tabular}




\begin{tabular}{|c|c|c|c|c|}
\hline $\begin{array}{l}\text { Skala zu non-mo- } \\
\text { torischen Sympto- } \\
\text { men Frage } 28 \\
\text { Schwere } \\
\text { keine/leicht/mä- } \\
\text { Big/schwer }\end{array}$ & $\begin{array}{l}(n=115) \\
51 / \quad 33 / \\
21 / 10 \\
(44,3 \% / \\
28,7 \% / \\
18,3 \% / \\
8,7 \%)\end{array}$ & $\begin{array}{l}(n=116) \\
107 / 5 / 2 / \\
2 \\
(92,2 \% / \\
4,3 \% / \\
1,7 \% / \\
1,7 \%)\end{array}$ & $\begin{array}{l}(n=100) \\
90 / 8 / 1 / \\
1 \\
(90 \% / \\
8 \% / 1 \% / \\
1 \%)\end{array}$ & $\begin{array}{l}(\mathrm{n}=100) \\
98 / 2 / 0 / \\
0 \\
(98 \% / \\
2 \% / 0 \% / \\
0 \%)\end{array}$ \\
\hline
\end{tabular}

\begin{tabular}{|c|c|c|c|c|}
\hline $\begin{array}{l}\text { Skala zu non-mo- } \\
\text { torischen Sympto- } \\
\text { men Frage } 28 \text { Häu- } \\
\text { figkeit } \\
\text { nie/selten/gelegent- } \\
\text { lich/häu- } \\
\text { fig/sehr/häufig }\end{array}$ & $\begin{array}{l}(n=115) \\
51 / 2 / 3 / \\
11 / 48 \\
(44,3 \% / \\
1,7 \% / \\
2,6 \% / \\
9,6 \% / \\
41,7 \%)\end{array}$ & $\begin{array}{l}(n=116) \\
107 / 1 / 2 / \\
1 / 5 \\
(92,2 \% / \\
0,9 \% / \\
1,7 \% / \\
0,9 \% / \\
4,3 \%)\end{array}$ & $\begin{array}{l}(n=100) \\
90 / 2 / 2 / \\
1 / 5 \\
(90 / 2 \% / \\
2 \% / 1 \% / \\
5 \%)\end{array}$ & $\begin{array}{l}98 / 1 / 0 / \\
0 / 1 \\
(98 / 1 \% / \\
0 \% / 0 \% / \\
1 \%)\end{array}$ \\
\hline
\end{tabular}

\begin{tabular}{|l|l|l|l|l|l|}
\hline Skala zu non-mo- & $(\mathrm{n}=115)$ & $(\mathrm{n}=116)$ & $(\mathrm{n}=100)$ & $(\mathrm{n}=100)$ & $<.001$ \\
torischen Sympto- & 3,37 & 0,45 & 0,41 & 0,05 & \\
$\begin{array}{l}\text { men Frage 28 HxS } \\
M(S D)\end{array}$ & $(3,83)$ & $(1,93)$ & $(1,61)$ & $(0,41)$ & \\
$M(S)$ & & & &
\end{tabular}

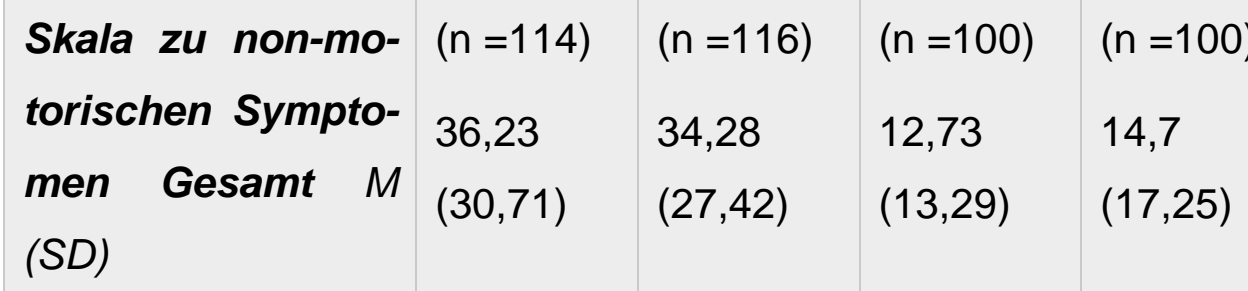

$\mathrm{PD}=$ Parkinsonpatienten, $\mathrm{KG}=$ Kontrollgruppe, $\mathrm{BL}=$ Baseline, $\mathrm{FU}=$ Folgeuntersuchung (Follow-up), $\mathrm{M=}$ Mittelwert, SD= Standardabweichung

Es zeigten sich bei den Patienten signifikante Unterschiede zwischen der BaselineTestung und der Folgeuntersuchung, ausgenommen die Gesamtwertung der Skala zu non-motorischen Symptomen zeigte keinen signifikanten Unterschied $(p=.429)$. Nach 24 Monaten gaben beispielsweise nur noch 13,2 \% der Patienten an ihren Geruchssinn als normal zu empfinden, in der Baseline-Testung empfanden dies 
noch mehr als doppelt so viele Patienten (35,65\%). Bei der Frage 2 des PD NMS Fragebogens gaben in der Folgeuntersuchung jedoch weniger Patienten (9,4\%) eine Veränderung oder einen Verlust des Riechens und Schmeckens an als in der Baseline-Testung (61,5 \%). Auch bei der Skala zu non-motorischen Symptomen Frage 28 sagten im Follow-up (92,2 \%) mehr Patienten aus keine Beschwerden zu haben als in der Baseline-Testung (44,3\%).

Die drei Testungen zur der subjektiven Wahrnehmung des Riechens stehen somit im Widerspruch zueinander.

\subsubsection{Objektive Riechtestungen zur Identifikation von 16 Qualitäten mithilfe der Sniffin' Sticks}

Identifikationstest 1- 16

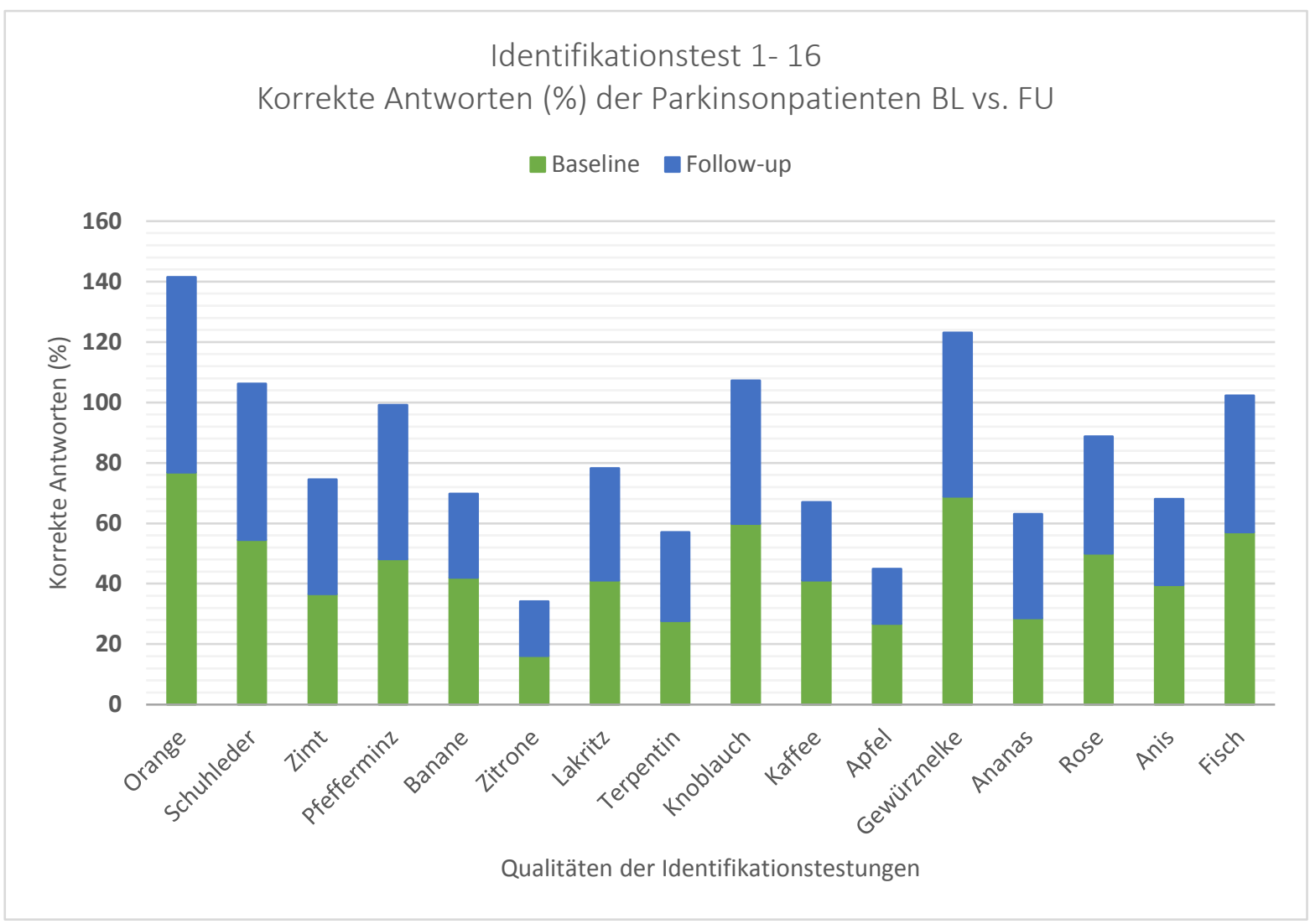

Abbildung 10 Identifikationstestung 1-16, Vergleich Baseline- und Folgeuntersuchung

Signifikante Unterschiede zwischen den Baseline Testungen und der Folgeuntersuchung zeigten bei den Patienten Test Nr.5-Banane $(p=.016)$, Nr.9-Knoblauch $(p=$ .047), Nr.10-Kaffee ( $p=.006)$, Nr.12-Gewürznelke $(p=.011)$, Nr.15-Anis $(p=.047)$ und Nr.16-Fisch $(p=.023)$. 
Bei den Kontrollpersonen unterschieden sich Test Nr.8-Terpentin $(p=.036)$ und Nr.10-Kaffee signifikant $(p=.036)$. Hier gaben die getesteten Personen abweichende Antworten an.

Tabelle 14 Korrekte Antworten im Identifikationstest 1-16 und Gesamt, Baseline- und Folgeuntersuchung, Signifikanz (p-Wert) BL vs. FU

\begin{tabular}{|c|c|c|c|c|c|c|}
\hline \multirow[t]{3}{*}{ Identifikationstest } & \multicolumn{2}{|c|}{ PD } & \multirow{3}{*}{$\begin{array}{l}\text { p-Wert } \\
\text { PD } \\
\text { BL vs. FU }\end{array}$} & \multicolumn{2}{|c|}{ HC } & \multirow{3}{*}{$\begin{array}{l}\text { p-Wert } \\
\text { KG } \\
\text { BL vs. FU }\end{array}$} \\
\hline & BL & FU & & BL & FU & \\
\hline & & & & & & \\
\hline Orange (1) & $\begin{array}{c}86 \\
(76,8 \\
\%)\end{array}$ & $\begin{array}{c}75 \\
(64,7 \%)\end{array}$ & .050 & $\begin{array}{c}90 \text { (90 } \\
\%)\end{array}$ & $\begin{array}{c}94 \\
(94 \%)\end{array}$ & .581 \\
\hline Schuhleder (2) & $\begin{array}{c}61 \\
(54,5 \\
\%)\end{array}$ & $\begin{array}{c}60 \\
(51,7 \%)\end{array}$ & .878 & $\begin{array}{l}74 \\
(74,7 \%)\end{array}$ & $\begin{array}{c}77 \\
(77 \%)\end{array}$ & .720 \\
\hline Zimt (3) & $\begin{array}{c}41 \\
(36,6 \\
\%)\end{array}$ & $\begin{array}{c}44 \\
(37,9 \%)\end{array}$ & .864 & $\begin{array}{c}61 \\
(61,6 \%)\end{array}$ & $\begin{array}{c}73 \\
(73 \%)\end{array}$ & .065 \\
\hline Pfefferminz (4) & $\begin{array}{c}54 \\
(48,2 \\
\%)\end{array}$ & $\begin{array}{c}59 \\
(50,9 \%)\end{array}$ & .755 & $\begin{array}{c}94 \\
(94,9 \%)\end{array}$ & $\begin{array}{c}92 \\
(92 \%)\end{array}$ & .508 \\
\hline Banane (5) & $\begin{array}{c}47 \\
(42 \%)\end{array}$ & $\begin{array}{c}32 \\
(27,8 \%)\end{array}$ & .016 & $\begin{array}{c}89 \\
(89,9 \%)\end{array}$ & $\begin{array}{c}85 \\
(85 \%)\end{array}$ & .388 \\
\hline Zitrone (6) & $\begin{array}{c}18 \\
(16,1 \\
\%)\end{array}$ & $\begin{array}{c}21 \\
(18,1 \%)\end{array}$ & .851 & $\begin{array}{c}38 \\
(38,4 \%)\end{array}$ & $\begin{array}{c}35 \\
(35 \%)\end{array}$ & .755 \\
\hline Lakritz (7) & $\begin{array}{c}46 \\
(41,1 \\
\%)\end{array}$ & $\begin{array}{c}43 \\
(37,1 \%)\end{array}$ & .627 & $\begin{array}{c}80 \\
(80,8 \%)\end{array}$ & $\begin{array}{c}88 \\
(88 \%)\end{array}$ & .115 \\
\hline
\end{tabular}




\begin{tabular}{|c|c|c|c|c|c|c|}
\hline Terpentin (8) & $\begin{array}{c}31 \\
(27,7 \\
\%)\end{array}$ & $\begin{array}{c}34 \\
(29,3 \%)\end{array}$ & .871 & $\begin{array}{c}53 \\
(53,5 \%)\end{array}$ & $\begin{array}{c}39 \\
(39 \%)\end{array}$ & .036 \\
\hline Knoblauch (9) & $\begin{array}{c}67 \\
(59,8 \\
\%)\end{array}$ & $\begin{array}{c}55 \\
(47,4 \%)\end{array}$ & .047 & $\begin{array}{c}81 \\
(81,8 \%)\end{array}$ & $\begin{array}{c}86 \\
(86 \%)\end{array}$ & .359 \\
\hline Kaffee (10) & $\begin{array}{c}46 \\
(41,1 \\
\%)\end{array}$ & $\begin{array}{c}30 \\
(25,9 \%)\end{array}$ & .006 & $\begin{array}{c}83 \\
(83,8 \%)\end{array}$ & $\begin{array}{c}71 \\
(71 \%)\end{array}$ & .036 \\
\hline Apfel (11) & $\begin{array}{c}30 \\
(26,8 \\
\%)\end{array}$ & $\begin{array}{c}21 \\
(18,1 \%)\end{array}$ & .163 & $\begin{array}{c}35 \\
(35,7 \%)\end{array}$ & $\begin{array}{c}36 \\
(36 \%)\end{array}$ & 1.000 \\
\hline Gewürznelke (12) & $\begin{array}{c}77 \\
(68,8 \\
\%)\end{array}$ & $\begin{array}{c}63 \\
(54,3 \%)\end{array}$ & .011 & $\begin{array}{c}92 \\
(93,9 \%)\end{array}$ & $\begin{array}{c}86 \\
(86 \%)\end{array}$ & .092 \\
\hline Ananas (13) & $\begin{array}{c}32 \\
(28,6 \\
\%)\end{array}$ & $\begin{array}{c}40 \\
(34,5 \%)\end{array}$ & .229 & $\begin{array}{c}59 \\
(60,2 \%)\end{array}$ & $\begin{array}{c}64 \\
(64 \%)\end{array}$ & .608 \\
\hline Rose (14) & $\begin{array}{c}56 \\
(50 \%)\end{array}$ & $\begin{array}{c}45 \\
(38,8 \%)\end{array}$ & .081 & $\begin{array}{c}84 \\
(85,7 \%)\end{array}$ & $\begin{array}{c}87 \\
(87 \%)\end{array}$ & .791 \\
\hline Anis (15) & $\begin{array}{c}44 \\
(39,6 \\
\%)\end{array}$ & $\begin{array}{c}33 \\
(28,4 \%)\end{array}$ & .047 & $\begin{array}{c}78 \\
(79,6 \%)\end{array}$ & $\begin{array}{c}87 \\
(87 \%)\end{array}$ & .152 \\
\hline Fisch (16) & $\begin{array}{c}64 \\
(57,1 \\
\%)\end{array}$ & $\begin{array}{c}52 \\
(45,2 \%)\end{array}$ & .023 & $\begin{array}{c}93 \\
(94,9 \%)\end{array}$ & $\begin{array}{c}92 \\
(92 \%)\end{array}$ & .687 \\
\hline Gesamt M (SD) & $\begin{array}{c}6,91 \\
(3,36)\end{array}$ & $\begin{array}{c}5,98 \\
(3,49)\end{array}$ & $<.001$ & $\begin{array}{l}11,91 \\
(2,55)\end{array}$ & $\begin{array}{l}11,87 \\
(2,52)\end{array}$ & .730 \\
\hline
\end{tabular}




\subsubsection{Identifikationstests Gesamt}

Die hier dargestellte Grafik zeigt die Entwicklung des Gesamtscores der Identifikationstestungen von der Baseline- bis zur Folgeuntersuchung. Es sind die Mittelwerte dargestellt.

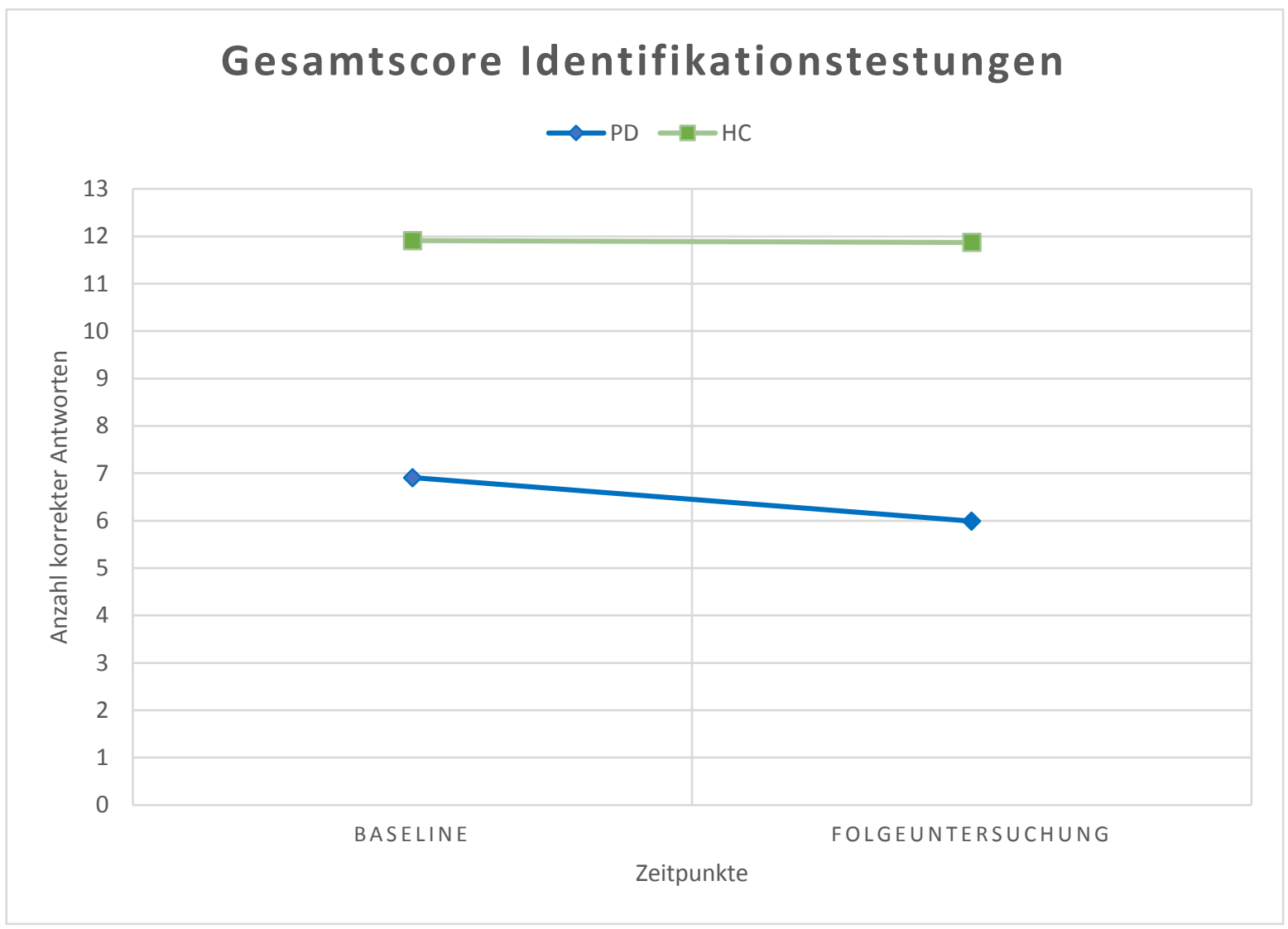

Abbildung 11 Gesamtscore der Identifikationstestungen, Baseline und Folgeuntersuchung

Während die Parkinsonpatienten in der Baseline-Testung einen Mittelwert von 6,91 Punkten zeigten, waren es in der Folgeuntersuchung im Mittel noch 5,99 Punkte. Hier liegt ein signifikanter Unterschied vor $(p<.001)$. Die Kontrollgruppe zeigte in der Baseline-Testung einen höheren Mittelwert (11,91 Punkte). In der Folgeuntersuchung erzielten sie im Mittel 11,87 Punkte. Es liegt kein signifikanter Unterschied vor $(p=.730)$. Die Patienten wiesen nach 24 Monaten einen signifikant schlechteren Riechtest auf als in der Baseline-Testung, für die Kontrollgruppe ließ sich das nicht nachweisen. 


\section{Diskussion}

\subsection{Demographische Daten}

Die in diese Studie miteinbezogenen 117 Patienten und 100 Kontrollpersonen zeigten bezogen auf das Alter, das Geschlecht und die Schulbildung, keine signifikanten Unterschiede, sodass ein Vergleich zulässig ist. Wie erwartet, waren die Testergebnisse des MDS UPDRS und der Hoehn \& Yahr-Klassifikation im Mittel in der Patientengruppe höher als bei der Kontrollgruppe. In allen Testungen der Folgeuntersuchungen zeigte sich darüber hinaus dem Krankheitsverlauf folgend eine progrediente Symptomatik. Dies ist anhand der ansteigenden Mittelwerte der MDS UPDRS Testergebnisse nachzuvollziehen. Über die Hälfte der Patienten (57,2 \%) befanden sich nach Hoehn und Yahr zum Zeitpunkt der ersten Datenerfassung in einem frühen Stadium der Erkrankung, in der die Symptome auf eine Körperhälfte beschränkt waren (Stadium 1 und 1,5). Nach 24 Monaten zeigten die Probanden deutlich niedrigere Testergebnisse. In der Folgeuntersuchung befanden sich lediglich 27,6\% in Stadium 1 und 1,5. Demgegenüber befanden sich über die Hälfte der Patienten (65,5\%) im Stadium 2, 2,5 und 3. Es fand also zu den fortgeschrittenen Stadien hin eine deutliche Verschiebung statt.

Die Dauer der Erkrankung zum Zeitpunkt der erstmaligen Datenerhebung 2012 lag bei den Patienten bei 23,7 Monaten. Dabei ist zu erwähnen, dass der Beginn der Erkrankung ein subjektiv wahrgenommener Zeitpunkt ist, der näherungsweise durch den Patienten festgelegt wird. Die maximale Zeitspanne lag bei 20 Jahren, die minimale bei zwei Monaten.

\subsection{Klinische Parameter}

Für die Erfassung einer bestehenden Depression wurde für die vorliegende Arbeit der GDS, BDI und MADRS durchgeführt. Sowohl für die Baseline- als auch für die Folgeuntersuchungen zeigten sich im Vergleich zwischen der Patientengruppe und der Kontrollgruppe hochsignifikante Unterschiede in allen Testungen $(p<.001)$. Die Patienten zeigten sowohl im GDS als auch im BDI und MADRS häufiger eine depressive Symptomatik als die Kontrollpersonen. Hierbei ist zu erwähnen, dass sich 
die Symptome einer Depression und die einer Parkinsonsymptomatik, wie beispielsweise Agitation, Müdigkeit, Appetitlosigkeit oder Hypomimie, überschneiden. Dies stellt bezüglich der Diagnosestellung eine Herausforderung dar (Schrag 2006). Die Lebensqualität der Patienten mit idiopathischem Parkinsonsyndrom wird jedoch entscheidend von mit der Erkrankung einhergehenden Symptomen, wie Depression, körperliche Beeinträchtigung und der Schwere der Erkrankung sowie durch kognitive Einschränkungen, beeinträchtigt (Schrag 2000). Um dies zu erfassen, wurde auch der PDQ-39-Test in die DeNoPa-Studie miteinbezogen. In den Testergebnissen des PDQ-39 spiegelt sich dabei die vorangegangene Hypothese wider. Die Patienten zeigten in den Baseline- und Folgeuntersuchungen im Mittel höhere Ergebnisse als die Kontrollgruppe, was auf eine schlechtere Lebensqualität durch Symptome, wie beispielsweise Depression, schließen lässt.

\subsection{Riechtestungen}

Die Diagnosestellung des idiopathischen Parkinsonsyndroms in einem frühen Stadium der Erkrankung ist von entscheidender Bedeutung. Es könnte dadurch ein maximaler Nutzen aus einer Therapie gezogen werden, die die Progression der Erkrankung aufhalten könnte (Bohnen et al. 2007).

\subsubsection{Objektive und subjektive Riechtestungen}

Die in der vorliegenden Arbeit präsentierten Ergebnisse lassen die Annahme zu, dass Parkinsonpatienten im Rahmen der Erkrankung frühzeitig eine Riechstörung entwickeln. Diese Vermutung trafen Doty et al. bereits 1992.

Die subjektiven und objektiven Riechtestungen zeigen alle hoch signifikante Unterschiede zwischen der Patientengruppe und der gesunden Kontrollgruppe. Sowohl die persönliche Einschätzung und die subjektiven Fragebögen (PD NMSFragebogen Frage 2 und Skala zu non-motorischen Symptomen Frage 28) als auch die Ergebnisse der objektiven Testungen mithilfe der Sniffin' Sticks (Schwellentest, Diskriminationstest und Identifikationstest) unterstreichen, dass die De-Novo-Parkinsonpatienten im Gegensatz zu der Kontrollgruppe größere Riechdefizite zeigen.

$64,35 \%$ der Patienten gaben in der persönlichen Einschätzung bereits an, eine verminderte Riechfunktion bemerkt zu haben, während dies im Vergleich nur $13 \%$ der 
Kontrollpersonen beobachteten. Auch in der Frage 2 des PD NMS Fragebogens, in der explizit nach Veränderungen beim Riechen und Schmecken gefragt wurde, beantworteten über die Hälfte der Patienten (61,5\%) und lediglich $10 \%$ der Kontrollpersonen die Frage mit „ja“. Auch die Skala zu non-motorischen Symptomen zeigte diese Tendenz. Wie bereits erwähnt, wurde die Frage 28, auf die hier Bezug genommen wird, in mehrere Abschnitte untergliedert. Es sollten Angaben über die Schwere und Häufigkeit des Riechdefizits gemacht werden. Daraus wurde die Summe und das Produkt gebildet. Mittelgradig schwere Einschränkungen zeigten $18,3 \%$ der Patienten und lediglich $1 \%$ der Kontrollgruppe. 41,7 \% der Patienten gaben sogar sehr häufige Beschwerden an, während dies nur $5 \%$ der Probanden in der Kontrollgruppe aufwiesen. Die objektiven Riechtests bestätigen die subjektiven Angaben. Auch hier zeigten alle Testungen hoch signifikante Unterschiede. Die Mittelwerte in allen drei Testungen (Schwellentest, Diskriminationstest, Identifikationstest) waren bei den Patienten niedriger als bei der Kontrollgruppe.

Damit einhergehend zeigt sich der TDI-Score, der bei $49 \%$ der Patienten eine Hyposmie und bei $41 \%$ eine Anosmie aufwies. Demzufolge beobachteten $90 \%$ der Patienten eine Beeinträchtigung des Riechens. Im Vergleich dazu zeigten nur $41 \%$ der Kontrollpersonen eine Einschränkung (39 \% Hyposmie, $2 \%$ Anosmie).

Auch in den errechneten Spearman Korrelationen zeigten sich für die objektiven Riechtestungen geringe bis hochgradige Zusammenhänge mit den subjektiven Bewertungen. Für den Gesamtbetrag des PD NMS Fragebogens zeigten sich geringgradige, negative Korrelationen, die Skala zu non-motorischen Symptomen korrelierte sogar zum Teil hochgradig. Für den Schwellentest zeigten sich bezogen auf die Schwere, Häufigkeit und deren Summe ein hochgradig negativer Zusammenhang. Zeigten die Patienten also in den subjektiven Fragebögen nach eigenen Beobachtungen Einschränkung des Geruchs, konnte dies auch in den objektiven Testungen durch den Schwellentest, Diskriminationstest und Identifikationstest mittels Sniffin' Sticks nachgewiesen werden.

Um die Entwicklung des Riechvermögens im Laufe der Erkrankung verfolgen zu können, wurden die Riechtestungen nach 24 Monaten erneut durchgeführt. Hierbei zeigte sich in den persönlichen Angaben zum Riechvermögen, dass 35,65 \% der Patienten zu Beginn der Studie subjektiv ein normales Riechvermögen angaben. Nach 24 Monaten lag diese Gruppe nur noch bei 13,2 \%. Die Anzahl der Patienten 
mit einem verminderten Riechvermögen nahm ebenfalls von $64,35 \%$ auf $86 \%$ zu. Es ließ sich demnach im Verlauf eine deutliche Abnahme des Riechvermögens nachweisen. Der Unterschied zwischen der Baseline- und der Folgeuntersuchung ist signifikant $(p<.001)$.

Bezugnehmend auf die Frage, ob eine Veränderung des Riechvermögens beobachtet wurde (PD NMS-Fragebogen Frage 2), nahm die Anzahl der positiv beantworteten Fragen von $61,5 \%$ auf $9,4 \%$ bei den Patienten ab. Dies lässt sich dadurch erklären, dass der Grad der Progression des Riechdefizits zu gering ist, als dass er subjektiv wahrnehmbar wäre. Die Skala zu non-motorischen Symptomen gab, bezogen auf die Schwere der Symptomatik, wider Erwarten in der Folgeuntersuchung bei 92,2\% der Patienten, im Vergleich zu der Baseline-Testung 44,3\%, keine Beschwerden an. 4,3\% gaben nur noch leichte Beschwerden (Baseline 28,7 \%), 1,7 $\%$ (Baseline 18,3 \%) mäßige und 1,7 \% (Baseline 8,7 \%) schwere Symptome an. Die gleiche Tendenz zeigte sich bei allen Testungen der Skala zu non-motorischen Symptomen.

Der Identifikationstest zeigte bei den Patienten für bestimmte Qualitäten einen signifikanten Unterschied zwischen der Baseline- und der Folgeuntersuchung. Dieser lag bei der Qualität Banane, Knoblauch, Kaffee, Gewürznelke, Anis und Fisch vor. Hier gaben demzufolge mit einem signifikanten Unterschied weniger Patienten eine korrekte Antwort als zum Beginn der Studie. Die Einschränkungen des Riechvermögens haben hier zugenommen. Auch im Gesamtscore des Identifikationstests wird eine Progression der Symptomatik bei den Patienten deutlich. Die Patienten zeigten hier signifikant schlechtere Ergebnisse als in der Baseline-Testung.

Bezogen auf die daraus zu ziehenden Schlussfolgerungen muss jedoch bedacht werden, dass eine Störung der Riechfunktion nicht krankheitsspezifisch für das idiopathische Parkinsonsyndrom ist. Das Symptom der Hyposmie tritt auch bei anderen neurodegenerativen Erkrankungen, wie bei der Lewy-Körper-Demenz (McShane et al. 2001), der Multiplen Systematrophie (MSA), Chorea Huntington, Morbus Alzheimer, dem Guam-Parkinson-Demenz-Komplex und Motoneuronenerkrankungen, auf (Hawkes 1999). Eine Unterscheidung zwischen der supranukleären Blickparese (PSP) und dem idiopathischen Parkinsonsyndrom scheint jedoch durch einen Riechtest aufgrund der normalen Riechfunktion bei der supranukleären Blickparese möglich zu sein (Doty et al. 1993). Des Weiteren ist laut Katzenschlager 
(2004) ein vaskuläres Parkinsonsyndrom aufgrund der normalen Riechfunktion ebenfalls auszuschließen. Deshalb kann eine potenziell frühzeitige Diagnosestellung nur mit der Unterstützung anderer diagnostischer Verfahren sichergestellt werden. Tissingh et al. (2001) schlägt die Kombination von Riechtests mit bildgebenden Verfahren wie dem SPECT oder dem PET-CT vor, um die Erkrankung im prämotorischen Stadium diagnostizieren zu können. Auch in einer Studie von Brooks (1998) wird das PET-CT oder das SPECT als sensitivste Methode zur Detektion von präklinisch vorliegender dopaminerger Dysfunktion und damit einer aktiven Parkinsonerkrankung bei Personen mit dem Risiko eine Parkinsonerkrankung zu bekommen, proklamiert. Dies entspricht jedoch einer kostenintensiven Methode, die nicht in allen Krankenhäusern verfügbar ist und demzufolge nicht standardmäßig durchführbar ist. Des Weiteren ist eine Unterscheidung zwischen dem idiopathischen Parkinsonsyndrom, der Multiplen Systematrophie (MSA) und der Progressiven supranukleären Blickparese (PSP) nicht möglich (Mollenhauer und Zhang 2012). Sommer et al. (2004) schlagen vor, Patienten mit einer olfaktorischen Dysfunktion zusätzlich einer transkraniellen Sonographie zu unterziehen, um Patienten mit dem Risiko ein idiopathisches Parkinsonsyndrom zu entwickeln, zu identifizieren. Die transkranielle Sonographie ist im Vergleich kosteneffizienter, weniger invasiv und einer größeren Anzahl an Krankenhäusern zugänglich (Schapira 2013).

Auch Montgomery et al. (2000) schreibt, dass eine Verbesserung der prädiktiven Aussagekraft durch die Kombination von unabhängigen Tests gewährleistet werden kann. Für die von inm benannte Testbatterie schlägt er die Kombination von olfaktorischen Tests, Testungen zu Depression (BDI) und der Motorik (Bewegung des Handgelenkes) vor. Diese Kombination erzielte eine um $10 \%$ höhere Sensitivität (69 \%) bei der Unterscheidung zwischen Patienten mit einer milden Symptomatik und der Kontrollgruppe als die ausschließlich olfaktorische Testung. In den vorliegenden Daten korrelierte keine der getesteten Depressionsskalen (BDI, GDS, MADRS) mit den objektiven Riechtestungen. Es zeigten laut dem BDI insgesamt 25 Patienten eine Depression, davon lag eine leichte Depression bei 17 Patienten vor, eine mittelgradige bei 7 Patienten und eine schwere bei einem Patienten vor.

Des Weiteren muss ein Augenmerk darauf liegen, dass die Ergebnisse eines Riechtests auch von weiteren Faktoren beeinflusst werden können. So beschreiben Hedner et al. (2010) die Diskrimination und Identifikation eines Geruches als einen komplexen Vorgang, der eine gewisse kognitive Leistung erfordert. Betrachtet man die 
Korrelationen der vorliegenden Arbeit, zeigt sich dieser Zusammenhang, bezogen auf die Riechtestungen und die Testungen zur Kognition (Uhrentest, Mini-MentalState Test) auch. Im Uhrentest liegen bei den Patienten, bezogen auf den Diskriminationstest, geringgradige negative Korrelationen vor. Dementsprechend gehen geringe Werte im Diskriminationstest und somit ein geschwächter Geruchssinn mit hohen Werten im Uhrentest einher. Je höher der Wert im Uhrentest ausfällt, umso größer ist die kognitive Einschränkung. Der Schwellen- und Identifikationstest zeigt in Bezug auf den Uhrentest keine Korrelation. Der MMST korreliert geringgradig positiv mit dem Diskriminations- und dem Identifikationstest. Hohe Werte im MiniMental-State Test und eine dadurch ermittelte gute kognitive Leistung geht mit einem hohen Wert in den Riechtests einher. Da die Summe der korrekten Antworten bei den Riechtestungen gezählt wird, lässt sich daraus auf einen guten Geruchssinn schließen. Laut einer Studie von Kadastik-Eerme et al. (2016) ist dabei die kognitive Einschränkung unter den non-motorischen Symptomen des idiopathischen Parkinsonsyndroms das häufigste Symptom. Diese Feststellung unterstreicht die Relevanz dieses Problems.

Ein weiterer Faktor, der die Riechfunktion beeinflussen kann, ist das Alter. So beschreiben auch Daum et al. (2000) in ihrer Studie eine abnehmende Leistung des Riechvermögens mit zunehmendem Alter. Auch in den vorliegenden Daten zeigt sich hierfür ein Zusammenhang. Das Alter korreliert sowohl bei den Patienten als auch bei der Kontrollgruppe gering- bis mittelgradig negativ mit den drei objektiven Riechtestungen. Mit zunehmendem Alter zeigt sich demnach ein geringerer Wert in den Riechtestungen und damit ein abgeschwächter Geruchssinn. Krishnan et al. (2011) postulieren hingegen, dass auf alle non-motorischen Symptome bezogen, in einer auf das Alter angeglichenen Gruppe die Parkinsonpatienten häufiger non- motorische Symptome zeigen und diese schwerwiegender sind.

Im Vergleich lässt sich laut Boesveldt et al. (2008) und Daum et al. (2000) durch den Identifikationstest häufiger eine Riechstörung nachweisen als durch den Diskriminationstest. Demnach eignet sich der Identifikationstest besser zur Unterscheidung zwischen den Parkinsonpatienten und der Kontrollgruppe. In den vorliegenden Daten erreichten die Sensitivitäts- und Spezifitätswerte im Identifikationstest zum Teil Werte > 90 \%. Im Vergleich zu dem Schwellentest $(0,816)$ und dem Diskriminationstest $(0,809)$ zeigte sich für diese Fragestellung die größte Fläche unter der 
Kurve (AUC) in der ROC-Kurve im Identifikationstest mit 0,878. Dabei lässt die Fläche unter der Kurve Rückschlüsse über die Güte eines Tests ziehen. Je näher der Wert an +1 liegt, umso größer ist die Aussagekraft des Tests. Die vorliegenden Ergebnisse würden diese Hypothese demnach unterstreichen.

Darüber hinaus stellte Tissingh et al. (2001) für den Diskriminationstest einen Zusammenhang mit dem Erkrankungsstadium fest. Mit voranschreitendem Stadium der Erkrankung, nimmt die Riechfunktion ab. Diese Annahme wird durch die vorliegenden Daten gestützt. Der MDS UPDRS Abschnitt II, III und die Gesamtsumme korrelierten dabei geringgradig negativ mit den Riechtestungen. Je höher die Punktzahl des MDS UPDRS und damit je gravierender die Symptomatik, umso geringer sind die Testergebnisse in den Riechtestungen und die Leistung des Riechvermögens.

Die Diagnosestellung „Hyposmie“ stellt aufgrund der Tatsache, dass die Patienten das Riechdefizit selbst häufig nicht wahrnehmen und aufgrund mangelnder standardisierter ökonomischer Testverfahren ein Problem dar. Des Weiteren wird die Prüfung des Riechvermögens in der neurologischen Untersuchung oft vernachlässigt (Daum et al. 2000). Umso wichtiger ist es, die Testverfahren, die eine Hyposmie nachweisen können, zu optimieren. Um präzisere diagnostische Riechtests zu entwickeln, muss also das genaue Schemata der Hyposmie bei Parkinsonpatienten, verglichen mit der Bewertung der gewöhnlichen Riechfunktion, bekannt sein (Bohnen et al. 2007). Ein erster Schritt wäre beispielsweise die Optimierung der Riechtestungen mittels Sniffin' Sticks für Parkinsonpatienten. Dafür wurde in der vorliegenden Arbeit für die Riechtestungen der Patienten die Sensitivität, die Spezifität und der Youden-Index bestimmt. Der Youden-Index, der ein Maß für die Testgüte darstellt, lag für die Qualität „Banane“ am höchsten (0,4794). Der Geruch „Banane“ hat von den 16 getesteten Geruchsstoffen demnach am besten die Erkrankten von den gesunden Probanden unterschieden. Die Qualität „Pfefferminz" schnitt dabei mit 0,4674 am zweitstärksten ab, nachfolgend mit einem Youden-Index von 0,4277 zeigte sich der Geruchsstoff „Kaffee“ in der vorliegenden Studie. Daum et al. (2000) gab in seiner Studie, in der die Testungen auch mithilfe der Sniffin' Sticks durchgeführt wurden, in absteigender Stärke „Lakritz“, „Anis“, „Ananas“, „Apfel“, „Terpentin“ und „Banane" als die Qualitäten mit der größten diskriminatorischen Aussagekraft an. Auch in der hier vorliegenden statistischen Auswertung zeigte die Qualität „Apfel" mit 73,21\% und „Terpentin“ mit 72,32 \% die zweit- und dritthöchste Sensitivität. 
Die höchste Sensitivität wurde hier jedoch durch die Qualität „Zitrone“ mit 83,93 \% vertreten. Auch in der Studie von Double et al. (2003) wird berichtet, dass die Qualitäten „Banane“, „Ananas“, „Benzin“, „Rauch“ und „Zimt“ zwischen der Patientenund Kontrollgruppe diskriminieren und es somit möglich ist einen selektiveren Riechtest für Parkinsonpatienten zu entwickeln. Hier wurden die Testungen jedoch durch den UPSIT (University of Pennsylvania Smell Identification Test) und lediglich mit 12 Qualitäten durchgeführt. Hawkes und Shephard (1993) verwendeten für ihre Testungen ebenfalls den UPSIT, jedoch wurden hier 40 Gerüche in die Studie miteinbezogen. Die höchste Sensitivität und Spezifität zeigten hier die Qualitäten „Pizza“ und „Wintergrün“. Die Daten sind demzufolge nur limitiert vergleichbar, da diese „Pizza“ und „Wintergrün“ in der für die vorliegende Studie verwendeten Gerüche nicht vorkommen.

Die durch James Parkinson 1817 verfassten Beobachtungen zu der nach ihm benannten Erkrankung sind somit nur zum Teil korrekt, betrachtet man die kognitiven und olfaktorischen Einschränkungen, die im Laufe der Erkrankung auftreten. 


\section{ZUSAMMENFASSUNG}

Morbus Parkinson ist nach Morbus Alzheimer die am zweithäufigsten auftretende neurodegenerative Erkrankung. Die Diagnosestellung beruht zum jetzigen Zeitpunkt auf der klinischen Untersuchung und dem Ansprechen auf dopaminerge Medikamente, der Goldstandard ist jedoch nach wie vor die pathologische Untersuchung des Patienten post mortem. Ziel der derzeitigen Forschung ist es, durch die Detektion diagnostischer Biomarker eine frühzeitigere Diagnosestellung sowie die Entwicklung und Anwendung neuroprotektiver Therapien zu ermöglichen.

Bis dato existiert kein diagnostischer Biomarker für das idiopathische Parkinsonsyndrom und die einer Diagnose zu Grunde liegenden Symptome treten auch bei anderen Erkrankungen auf. Sie sind demnach nicht krankheitsspezifisch. Die Relevanz dieses Aspektes zeigt die hohe Anzahl an Fehldiagnosen, die in frühen Stadien des idiopathischen Parkinsonsyndroms gestellt werden. Diese kann bei Ärzten mit limitierter klinischer Erfahrung bei bis zu $25 \%$ liegen. Eine potentiell neuroprotektive Therapie könnte durch einen entsprechenden Biomarker zu einem wesentlich früheren Zeitpunkt eingesetzt werden und der dadurch denkbar veränderte Krankheitsverlauf gemessen werden (Miller und O'Callaghan 2015). Dabei muss bedacht werden, dass für verschiedene Typen der Parkinsonerkrankung eventuell unterschiedliche Biomarker nötig sind, weshalb eine Kombination mehrerer Marker der entscheidende Punkt zu sein scheint (Mollenhauer und Zhang 2012).

In der vorliegenden Arbeit wurden zur Detektion diagnostischer Biomarker an 117 De-Novo-Parkinson-Patienten und 100 Kontrollpersonen vielseitige Testungen, vor allem bezüglich non-motorischer Symptome, durchgeführt und ausgewertet. Insbesondere die Riechtestungen mithilfe der Sniffin' Sticks (Hummel et al. 1997) sollten in dieser Arbeit im Vordergrund stehen. Dabei konnten in den objektiven Identifikationstestungen in der Gruppe der Patienten für bestimmte Qualitäten hohe Sensitivitäten und Spezifitäten nachgewiesen werden und die bei den Parkinsonpatienten vorliegende Riechstörung spezifiziert werden. Die höchsten Sensitivitäten erzielten dabei in absteigender Reihenfolge die Qualitäten Zitrone (83,93 \%), Apfel (73,21\%) und Terpentin (72,32\%), diese konnten demnach von den 16 getesteten Qualitäten am besten die erkrankten Personen hervorheben. Pfefferminz (94,95 \%), Gewürznelke $(93,88 \%)$ und Orange $(90,91 \%)$ zeichneten sich durch die höchsten Spezifi- 
tätswerte aus. Im direkten Vergleich der drei objektiv durchgeführten Riechtestungen zur Schwelle, Diskrimination und Identifikation von Gerüchen kann bei allen durch die ermittelte Area Under The Curve von $>0,8$, von einer guten Testgüte gesprochen werden. Den höchsten Wert erzielte jedoch der Identifikationstest mit 0,878 .

Die vorliegende Arbeit kann darüber hinaus eine Aussage über den Verlauf des Riechvermögens der Probanden treffen, da zu vergleichende Daten aus der Folgeuntersuchung nach 24 Monaten miteinbezogen wurden. In der Folgeuntersuchung konnte ein progredient zunehmendes Riechdefizit bei den Parkinsonpatienten nachgewiesen werden. Der Gesamtscore des Identifikationstests, der aus den korrekt beantworteten Fragen der 16 Identifikationstestungen errechnet wird, zeigt hier eine signifikante Abnahme von im Mittel 6,91 auf 5,99 nach 24 Monaten. Das Riechvermögen der Patienten hat demnach im Vergleich zu der ersten Messung signifikant abgenommen, dies ließ sich für die Kontrollgruppe nicht nachweisen.

Die idiopathische Hyposmie scheint demnach ein präsymptomatisches, non-motorisches Symptom des Morbus Parkinson darzustellen, das im Laufe der Erkrankung zunimmt. Somit könnte das olfaktorische Defizit zukünftig einen Teil eines diagnostischen Biomarkers ausmachen. Als Screening für das idiopathische Parkinsonsyndrom dürfen nicht ausschließlich Riechtestungen durchgeführt werden, da die Hyposmie auch aus anderen Erkrankung, wie beispielsweise viralen Infekten, resultieren kann (Ponsen et al. 2010). Der Schlüssel zur Erfassung von Parkinsonerkrankten in einem früheren Stadium wird demnach die Kombination von mehreren klinischen Testungen sein, von denen die Prüfung des Riechvermögens ein Teil sein kann. Die Erfassung dieses olfaktorischen Defizits kann kostengünstig und binnen kurzer Zeit mittels Sniffin' Sticks getestet werden. Deshalb sollte die Prüfung der olfaktorischen Funktion standardmäßig differenzialdiagnostisch bei neurodegenerativen motorischen Erkrankung eingesetzt werden und weiterhin ein Bestandteil zukünftiger wissenschaftlicher Untersuchungen zur Erfassung diagnostischer Biomarker der idiopathischen Parkinsonerkrankung sein. 


\section{LITERATURVERZEICHNIS}

Adler CH (2011): Premotor symptoms and early diagnosis of Parkinson's disease. The Int J of Neurosci $\underline{121}$ (Suppl 2), 3-8

Alves G, Forsaa EB, Pedersen KF, Dreetz GM, Larsen JP (2008): Epidemiology of Parkinson's disease. J Neurol 255 (Suppl 5), 18-32

Beck AT, Ward CH, Mendelson M, Mock J, Erbaugh J (1961): An inventory for measuring depression. Arch Gen Psychiatry. 4, 561-571

Becker G, Muller A, Braune S, Buttner T, Benecke R, Greulich W, Klein W, Mark G, Rieke J, Thumler R (2002): Early diagnosis of Parkinson's disease. J Neurol 249 (Suppl 3), 3-8

Berardelli A, Wenning GK, Antonini A, Berg D, Bloem BR, Bonifati V, Brooks D, Burn DJ, Colosimo C, Fanciulli A et al. (2013): EFNS/MDS-ES recommendations for the diagnosis of Parkinson's disease. European Journal of Neurology 20, 16-34

Berendse HW, Booij J, Francot CM, Bergmans PL, Hijman R, Stoof JC, Wolters EC (2001): Subclinical dopaminergic dysfunction in asymptomatic Parkinson's disease patients' relatives with a decreased sense of smell. Annals of Neurology $\underline{50}, 34-41$

Biomarkers Definitions Working Group (2001): Biomarkers and surrogate endpoints: preferred definitions and conceptual framework. Clin Pharmacol Ther $\underline{69}$, 89-95

Bloem BR, Hausdorff JM, Visser JE, Giladi N (2004): Falls and freezing of gait in Parkinson's disease: A review of two interconnected, episodic phenomena. Mov Disord 19, 871-884

Boesveldt S, Verbaan D, Knol DL, Visser M, van Rooden SM, van Hilten JJ, Berendse HW (2008): A comparative study of odor identification and odor discrimination deficits in Parkinson's disease. Mov Disord 23, 1984-1990

Bohnen NI, Gedela S, Kuwabara H, Constantine GM, Mathis CA, Studenski SA, Moore RY (2007): Selective hyposmia and nigrostriatal dopaminergic denervation in Parkinson's disease. J Neurol $\underline{254}, 84-90$

Braak H, Del Tredici K, Rub U, Vos RAI de, Jansen Steur ENH, Braak E (2003): Staging of brain pathology related to sporadic Parkinson's disease. Neurobiol Aging 24, 197-211

Braak H, Ghebremedhin E, Rub U, Bratzke H, Del Tredici K (2004): Stages in the development of Parkinson's disease-related pathology. Cell and tissue research $\underline{318}, 121-134$

Brooks DJ (1998): The early diagnosis of parkinson's disease. Ann Neurol. 44 , S10-S18

Chaudhuri KR, Martinez-Martin P, Schapira AH, Stocchi F, Sethi K, Odin P, Brown RG, Koller W, Barone $P$, MacPhee $G$ et al. (2006): International multicenter pilot study of the first comprehensive self-completed nonmotor symptoms questionnaire for Parkinson's disease: The NMSQuest study. Mov Disord. 21, 916-923

Chaudhuri KR, Martinez-Martin P, Brown RG, Sethi K, Stocchi F, Odin P, Ondo W, Abe K, MacPhee $G$, MacMahon D et al. (2007): The metric properties of a novel non-motor symptoms scale for Parkinson's disease: Results from an international pilot study. Mov Disord 22, 1901-1911

Chen H, Burton EA, Ross GW, Huang X, Savica R, Abbott RD, Ascherio A, Caviness JN, Gao X, Gray KA et al. (2013): Research on the Pre-Motor Symptoms of Parkinson's Disease: Clinical and Etiological Implications. Environ Health Perspect 121(11-12), 1245-1252

Connolly BS, Lang AE (2014): Pharmacological Treatment of Parkinson Disease. JAMA $\underline{311}, 1670$ 
Daum RF, Sekinger B, Kobal G, Lang CJ (2000): Riechprüfung mit "sniffin' sticks" zur klinischen Diagnostik des Morbus Parkinson. Nervenarzt $\underline{71}$, 643-650

DGN: Deutsche Gesellschaft für Neurologie: Leitlinien für Diagnostik und Therapie in der Neurologie. Hrsg. von der Kommission "Leitlinien der Deutschen Gesellschaft für Neurologie" und DIner HC, Putzki N. 4. Auflage; Thieme, Stuttgart 2008

DGN: Deutsche Gesellschaft für Neurologie (2016): S3-Leitlinie: Idiopathisches Parkinsonsyndrom, 01.01.2016: http://www.dgn.org/images/red_leitli-

nien/LL_2016/PDFs_Download/030010_LL_langfassung_ips_2016.pdf

Dorsey ER, Constantinescu R, Thompson JP, Biglan KM, Holloway RG, Kieburtz K, Marshall FJ, Ravina BM, Schifitto G, Siderowf A et al. (2007): Projected number of people with Parkinson disease in the most populous nations, 2005 through 2030. Neurology $\underline{68}, 384-386$

Doty RL, Riklan M, Deems DA, Reynolds C, Stellar S (1989): The olfactory and cognitive deficits of parkinson's disease: Evidence for independence. Ann Neurol 25, $166-171$

Doty RL, Stern MB, Pfeiffer C, Gollomp SM, Hurtig HI (1992): Bilateral olfactory dysfunction in early stage treated and untreated idiopathic Parkinson's disease. J Neurol Neurosurg Psychiatry $\underline{55}, 138-142$

Doty RL, Golbe LI, McKeown DA, Stern MB, Lehrach CM, Crawford D (1993): Olfactory testing differentiates between progressive supranuclear palsy and idiopathic Parkinson's disease. Neurology 43,962

Double KL, Rowe DB, Hayes M, Chan DKY, Blackie J, Corbett A, Joffe R, Fung VS, Morris J, Halliday GM (2003): Identifying the pattern of olfactory deficits in Parkinson disease using the brief smell identification test. Archives of neurology $\underline{60}, 545-549$

Erro R, Picillo M, Vitale C, Amboni M, Moccia M, Longo K, Cozzolino A, Giordano F, Rosa A de, Michele $G$ de et al. (2013): Non-motor symptoms in early Parkinson's disease: a 2-year follow-up study on previously untreated patients. J Neurol Neurosurg Psychiatry $\underline{84}, 14-17$

Fahn S (2003): Description of Parkinson's disease as a clinical syndrome. Ann NY Acad Sci 991, 114

Fahn S (2015): The medical treatment of Parkinson disease from James Parkinson to George Cotzias. Mov Disord 30, 4-18

Folstein MF, Folstein SE, McHugh PR (1975): “Mini-mental state”. J Psychiat Res $\underline{12}$, 189-198

Gaenslen A, Swid I, Liepelt-Scarfone I, Godau J, Berg D (2011): The patients' perception of prodromal symptoms before the initial diagnosis of Parkinson's disease. Mov Disord 26, 653-658

Goetz CG, Fahn S, Martinez-Martin P, Poewe W, Sampaio C, Stebbins GT, Stern MB, Tilley BC, Dodel R, Dubois B et al. (2007): Movement Disorder Society-sponsored revision of the Unified Parkinson's Disease Rating Scale (MDS-UPDRS): Process, format, and clinimetric testing plan. Movement disorders official journal of the Movement Disorder Society 22, 41-47

Gonera EG, Hof MV, Berger HJC, van Weel C, Horstink, Martin WIM (1997): Symptoms and duration of the prodromal phase in parkinson's disease. Mov Disord 12, 871-876

Haehner A, Hummel T, Hummel C, Sommer U, Junghanns S, Reichmann H (2007): Olfactory loss may be a first sign of idiopathic Parkinson's disease. Mov Disord 22, 839-842

Harding AJ (2002): Clinical correlates of selective pathology in the amygdala of patients with Parkinson's disease. Brain $\underline{125}, 2431-2445$ 
Haugen J, Muller ML, Kotagal V, Albin RL, Koeppe RA, Scott PJ, Frey KA, Bohnen NI (2016): Prevalence of impaired odor identification in Parkinson disease with imaging evidence of nigrostriatal denervation. J neural transm $\underline{123}, 421-424$

Hawkes CH (1999): Is Parkinson's disease a primary olfactory disorder? QJM $\underline{92}, 473-480$

Hawkes CH, Shephard BC (1993): Selective anosmia in Parkinson's disease? Lancet 341, 435-436

Hedner M, Larsson M, Arnold N, Zucco GM, Hummel T (2010): Cognitive factors in odor detection, odor discrimination, and odor identification tasks. Journal of clinical and experimental neuropsychology $\underline{32}, 1062-1067$

Hely MA, Morris JG, Reid WG, Trafficante R (2005): Sydney Multicenter Study of Parkinson's disease: non-L-dopa-responsive problems dominate at 15 years. Movement disorders official journal of the Movement Disorder Society 20, 190-199

Herting B, Schulze S, Reichmann H, Haehner A, Hummel T (2008): A longitudinal study of olfactory function in patients with idiopathic Parkinson's disease. J Neurol 255, 367-370

Hoehn MM, Yahr MD (2001): Parkinsonism: onset, progression, and mortality. 1967. Neurology $\underline{57}, 26$

Hughes AJ, Daniel SE, Kilford L, Lees AJ (1992): Accuracy of clinical diagnosis of idiopathic Parkinson's disease: A clinico-pathological study of 100 cases. J Neurol Neurosurg Psychiatry $\underline{55}, 181-$ 184

Huisman E, Uylings HB, Hoogland PV (2004): A 100\% increase of dopaminergic cells in the olfactory bulb may explain hyposmia in Parkinson's disease. Movement disorders official journal of the Movement Disorder Society 19, 687-692

Hummel T, Sekinger B, Wolf SR, Pauli E, Kobal G (1997): 'Sniffin' Sticks': Olfactory Performance Assessed by the Combined Testing of Odour Identification, Odor Discrimination and Olfactory Threshold. Chem Senses 22, 39-52

Hummel T, Kobal G, Gudziol H, Mackay-Sim A (2007): Normative data for the "Sniffin' Sticks" including tests of odor identification, odor discrimination, and olfactory thresholds: an upgrade based on a group of more than 3,000 subjects. EurArch Otorhinoaryngol 264, 237-243

Jankovic J (2008): Parkinson's disease: clinical features and diagnosis. J Neurol Neurosurg Psychiatry $\underline{79}, 368-376$

Kadastik-Eerme L, Muldmaa M, Lilles S, Rosenthal M, Taba N, Taba P (2016): Nonmotor Features in Parkinson's Disease: What Are the Most Important Associated Factors? Parkinson's disease $\underline{2016}, 4370674$

Kalia LV, Lang AE (2015): Parkinson's disease. Lancet $\underline{386}$, 896-912

Katzenschlager R (2004): Olfactory function distinguishes vascular parkinsonism from Parkinson's disease. J Neurol Neurosurg Psychiatry $\underline{75}$, 1749-1752

Klein C (2006): Implications of genetics on the diagnosis and care of patients with Parkinson disease. Arch Neurol $\underline{63}, 328-334$

Kleiner-Fisman G, Herzog J, Fisman DN, Tamma F, Lyons KE, Pahwa R, Lang AE, Deuschl G (2006): Subthalamic nucleus deep brain stimulation: Summary and meta-analysis of outcomes. Mov Disord 21, S290-S304 
Krishnan S, Sarma G, Sarma S, Kishore A (2011): Do nonmotor symptoms in Parkinson's disease differ from normal aging? Movement disorders official journal of the Movement Disorder Society 26, $2110-2113$

Mayeux R, Marder K, Cote L, Denaro J, Hemenegildo N, Mejia H, Tang MX, Lantigua R, Wilder D, Gurland B (1995): The frequency of idiopathic Parkinson's disease by age, ethnic group, and sex in northern Manhattan, 1988-1993. Am J Epidemiol 142, 820-827

McShane RH, Nagy Z, Esiri MM, King E, Joachim C, Sullivan N, Smith AD (2001): Anosmia in dementia is associated with Lewy bodies rather than Alzheimer's pathology. J Neurol Neurosurg Psychiatry $\underline{70}, 739-743$

Miller DB, O'Callaghan JP (2015): Biomarkers of Parkinson's disease: present and future. Metabolism: clinical and experimental $\underline{64}, 6$

Mittmann N (1997): Montgomery-Asberg severity gradations. Am J Psychiatry 154, 1320b-1321

Mollenhauer B, Zhang J (2012): Biochemical premotor biomarkers for Parkinson's disease. Movement disorders official journal of the Movement Disorder Society 27, 644-650

Mollenhauer B, Locascio JJ, Schulz-Schaeffer W, Sixel-Döring F, Trenkwalder C, Schlossmacher MG (2011): $\alpha$-Synuclein and tau concentrations in cerebrospinal fluid of patients presenting with parkinsonism: A cohort study. Lancet Neurology 10, 230-240

Mollenhauer B, Trautmann E, Sixel-Doring F, Wicke T, Ebentheuer J, Schaumburg M, Lang E, Focke NK, Kumar KR, Lohmann K et al. (2013): Nonmotor and diagnostic findings in subjects with de novo Parkinson disease of the DeNoPa cohort. Neurology 81, 1226-1234

Montgomery E (2000): Early detection of probable idiopathic Parkinson's disease: I. development of a diagnostic test battery. Mov Disord, 467-473

Montgomery SA, Asberg M (1979): A new depression scale designed to be sensitive to change. Brit J Psychiat 134, 382-389

Müller MJ, Szegedi A, Wetzel H, Benkert O (2000): Moderate and severe depression. Journal of Affective Disorders $\underline{60}, 137-140$

Muntean ML, Sixel-Doring F, Trenkwalder C (2014): REM sleep behavior disorder in Parkinson's disease. J neural transm 121 , Suppl 141-47

Poewe W, Deuschl G: Diagnose und Differenzialdiagnose. In: Oertel W, Deuschl G, Poewe W (Hrsg.): Parkinson-Syndrome und andere Bewegungsstörungen. Thieme, Stuttgart 2012, 50-66

Parkinson J (2002): An essay on the shaking palsy. 1817. J Neuropsychiatry Clin Neurosci 14, 223236

Peto V, Jenkinson C, Fitzpatrick R (1998): PDQ-39: a review of the development, validation and application of a Parkinson's disease quality of life questionnaire and its associated measures. J Neurol $\underline{245}$ Suppl 1, 4

Ponsen MM, Stoffers D, Booij J, van Eck-Smit BLF, Wolters EC, Berendse HW (2004): Idiopathic hyposmia as a preclinical sign of Parkinson's disease. Ann Neurol $\underline{56}, 173-181$

Ponsen MM, Stoffers D, Twisk JW, Wolters EC, Berendse HW (2009): Hyposmia and executive dysfunction as predictors of future Parkinson's disease: A prospective study. Mov Disord 24, 10601065 
Ponsen MM, Stoffers D, Wolters E, Booij J, Berendse HW (2010): Olfactory testing combined with dopamine transporter imaging as a method to detect prodromal Parkinson's disease. J Neurol Neurosurg Psychiatry 81, 396-399

Postuma RB, Montplaisir J (2006): Potential early markers of Parkinson's disease in idiopathic rapid-eye-movement sleep behaviour disorder. Lancet Neurology $\underline{5}, 552-553$

Quinn N, Critchley P, Marsden CD (1987): Young onset Parkinson's disease. Movement disorders official journal of the Movement Disorder Society 2, 73-91

Reichmann H (2010): Clinical Criteria for the Diagnosis of Parkinson's Disease. Neurodegener Dis 7, 284-290

Ross GW, Petrovitch H, Abbott RD, Tanner CM, Popper J, Masaki K, Launer L, White LR (2008): Association of olfactory dysfunction with risk for future Parkinson's disease. Ann Neurol $\underline{63}, 167-173$

Schapira AH (2013): Recent developments in biomarkers in Parkinson disease. Current Opinion in Neurology 26, 395-400

Schrag A (2000): What contributes to quality of life in patients with Parkinson's disease? J Neurol Neurosurg Psychiatry $\underline{69}, 308-312$

Schrag A (2006): Quality of life and depression in Parkinson's disease. J Neurol Sci $\underline{248}, 151-157$

Schrag A, Jahanshahi M, Quinn N (2000): What contributes to quality of life in patients with Parkinson's disease? J Neurol Neurosurg Psychiatry $\underline{69}, 308-312$

Sixel-Döring F, Ebersbach G (2010): Nachsorge nach tiefer Hirnstimulation bei Patienten mit M. Parkinson. Nervenarzt $\underline{81}, 688-695$

Snijders AH, Nijkrake MJ, Bakker M, Munneke M, Wind C, Bloem BR (2008): Clinimetrics of freezing of gait. Movement disorders official journal of the Movement Disorder Society 23 Suppl 2 , S468-74

Sommer U, Hummel T, Cormann K, Mueller A, Frasnelli J, Kropp J, Reichmann H (2004): Detection of presymptomatic Parkinson's disease: combining smell tests, transcranial sonography, and SPECT. Movement disorders official journal of the Movement Disorder Society 19, 1196-1202

Spiegel J, Storch A, Jost WH (2006): Early diagnosis of Parkinson's disease. J Neurol 253 Suppl 4, 7

Stern MB, Marek KL, Friedman J, Hauser RA, LeWitt PA, Tarsy D, Olanow CW (2004): Double-blind, randomized, controlled trial of rasagiline as monotherapy in early Parkinson's disease patients. Movement disorders official journal of the Movement Disorder Society 19, 916-923

Stoof JC, Winogrodzka A, van Muiswinkel FL, Wolters EC, Voorn P, Groenewegen HJ, Booij J, Drukarch B (1999): Leads for the development of neuroprotective treatment in Parkinson's disease and brain imaging methods for estimating treatment efficacy. European journal of pharmacology $\underline{375}, 75-86$

Swanson LW, Petrovich GD (1998): What is the amygdala? Trends in Neurosciences $\underline{21}, 323-331$

Tarsy D (2012): Treatment of Parkinson Disease: A 64-Year-Old Man With Motor Complications of Advanced Parkinson Disease. JAMA 307, 2305-2314

Tissingh G, Berendse HW, Bergmans P, Dewaard R, Drukarch B, Stoof JC, Wolters E (2001): Loss of olfaction in de novo and treated Parkinson's disease: Possible implications for early diagnosis.

Mov Disord 16, 41-46 
Tolosa E, Pont-Sunyer C (2011): Progress in defining the premotor phase of Parkinson's disease. J Neurol Sci $\underline{310}, 4-8$

van Den Eeden SK (2003): Incidence of Parkinson's Disease: Variation by Age, Gender, and Race/Ethnicity. Am J Epidemiol 157, 1015-1022

Wenning GK, Shephard B, Hawkes C, Petruckevitch A, Lees A, Quinn N (1995): Olfactory function in atypical parkinsonian syndromes. Acta Neurologica Scandinavica $\underline{91}$, 247-250

Wirdefeldt K, Adami HO, Cole P, Trichopoulos D, Mandel J (2011): Epidemiology and etiology of Parkinson's disease: a review of the evidence. Eur J Epidemiol 26 Suppl 1, 58

Wolfensberger M, Schnieper I, Welge-Lussen A (2000): Sniffin'Sticks: a new olfactory test battery. Acta oto-laryngologica $\underline{120}, 303-306$

Yesavage JA, Brink TL, Rose TL, Lum O, Huang V, Adey M, Leirer VO. (1982-1983): Development and validation of a geriatric depression screening scale: a preliminary report. J Psychiatr Res., 3749

Youden WJ (1950): Index for rating diagnostic tests. Cancer $\underline{3}$, 32-35 


\section{ANHANG}
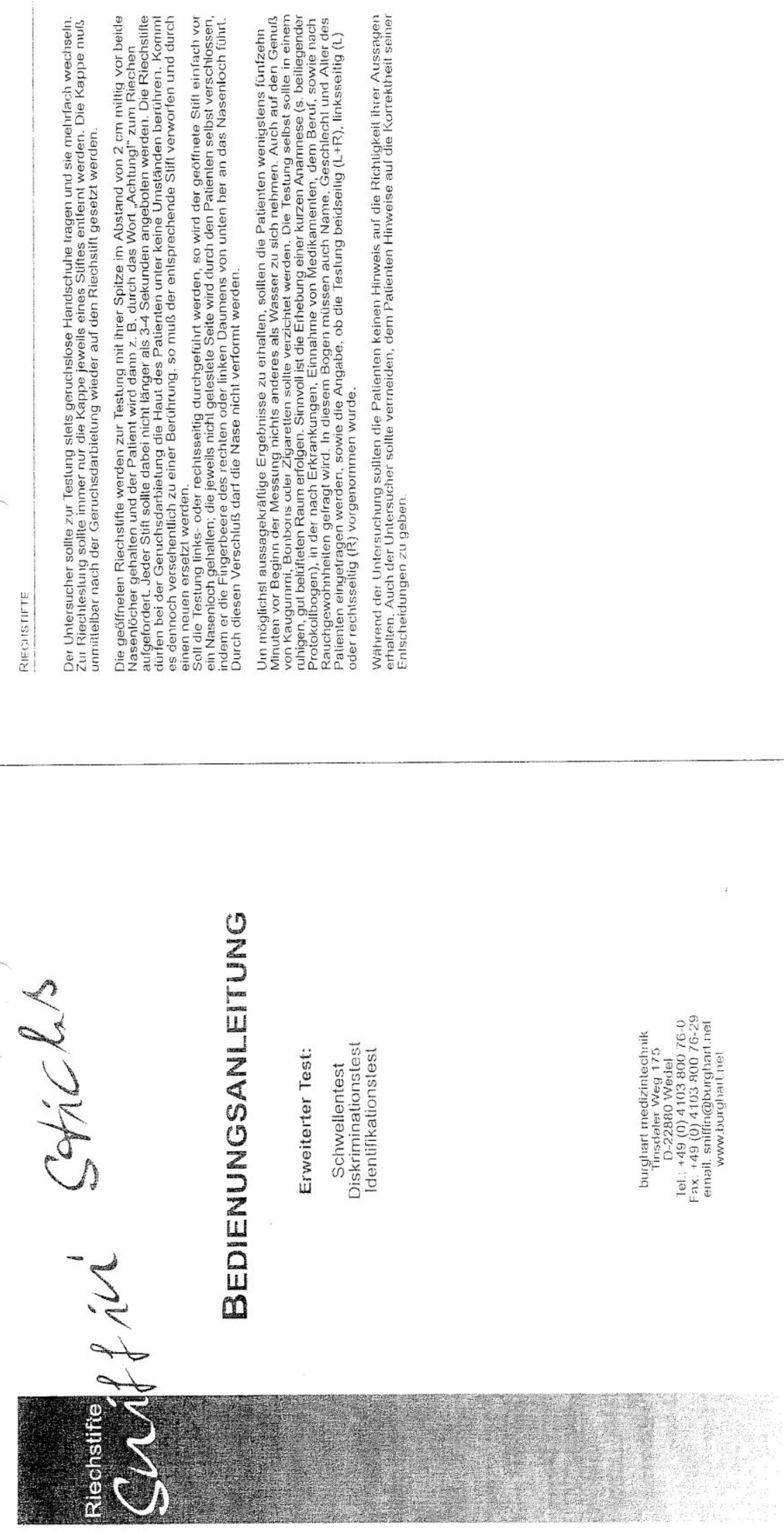

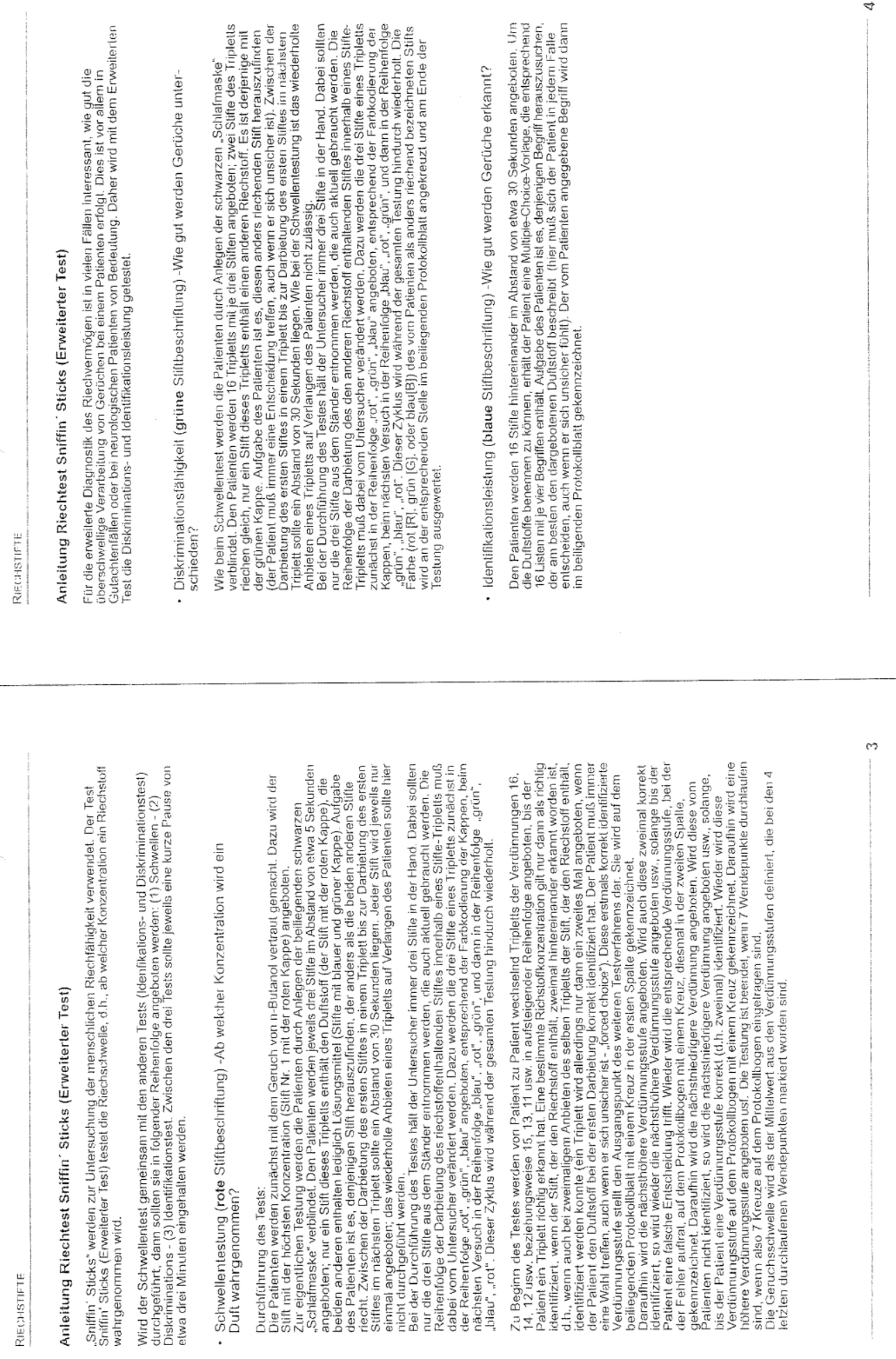

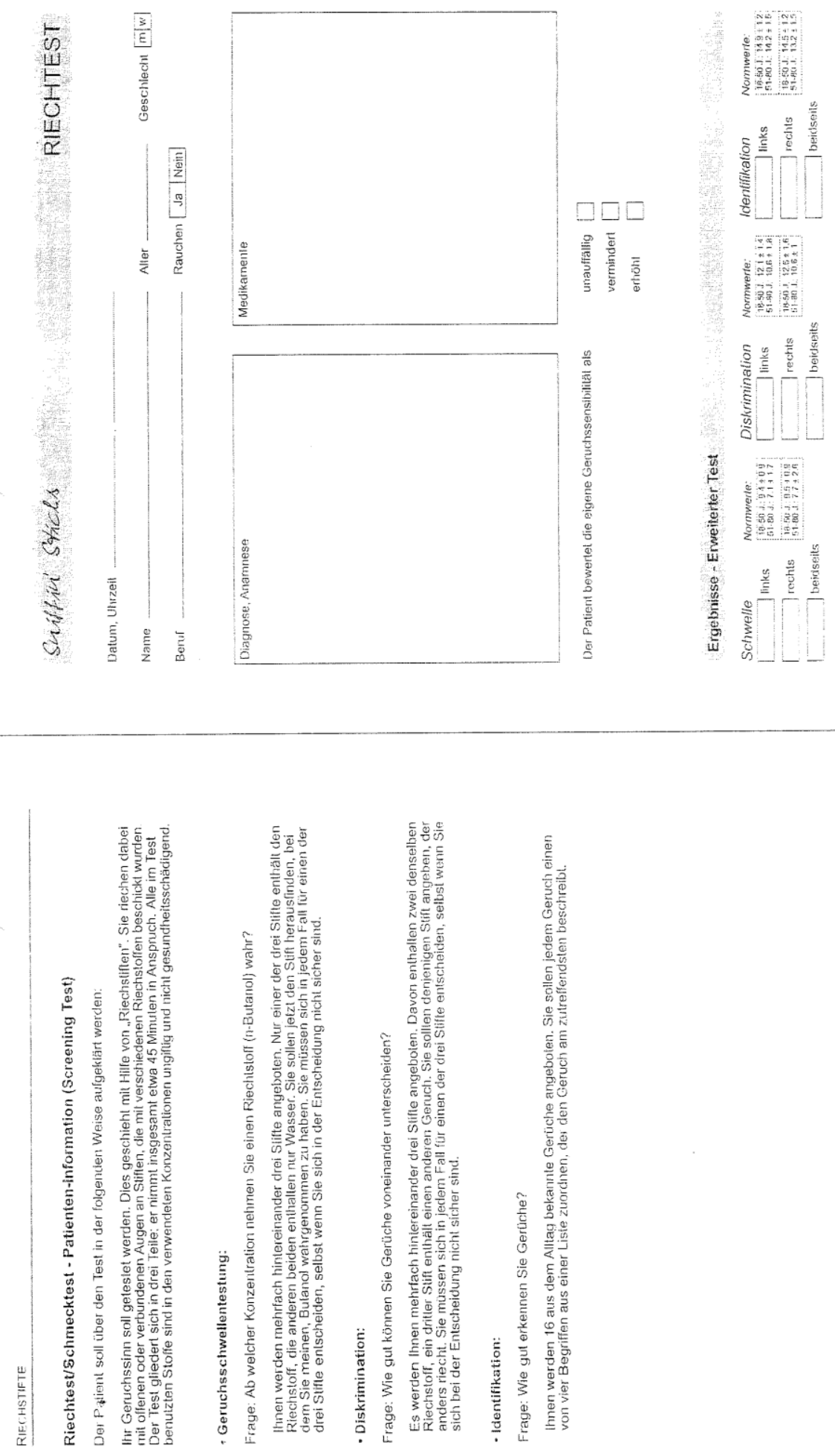

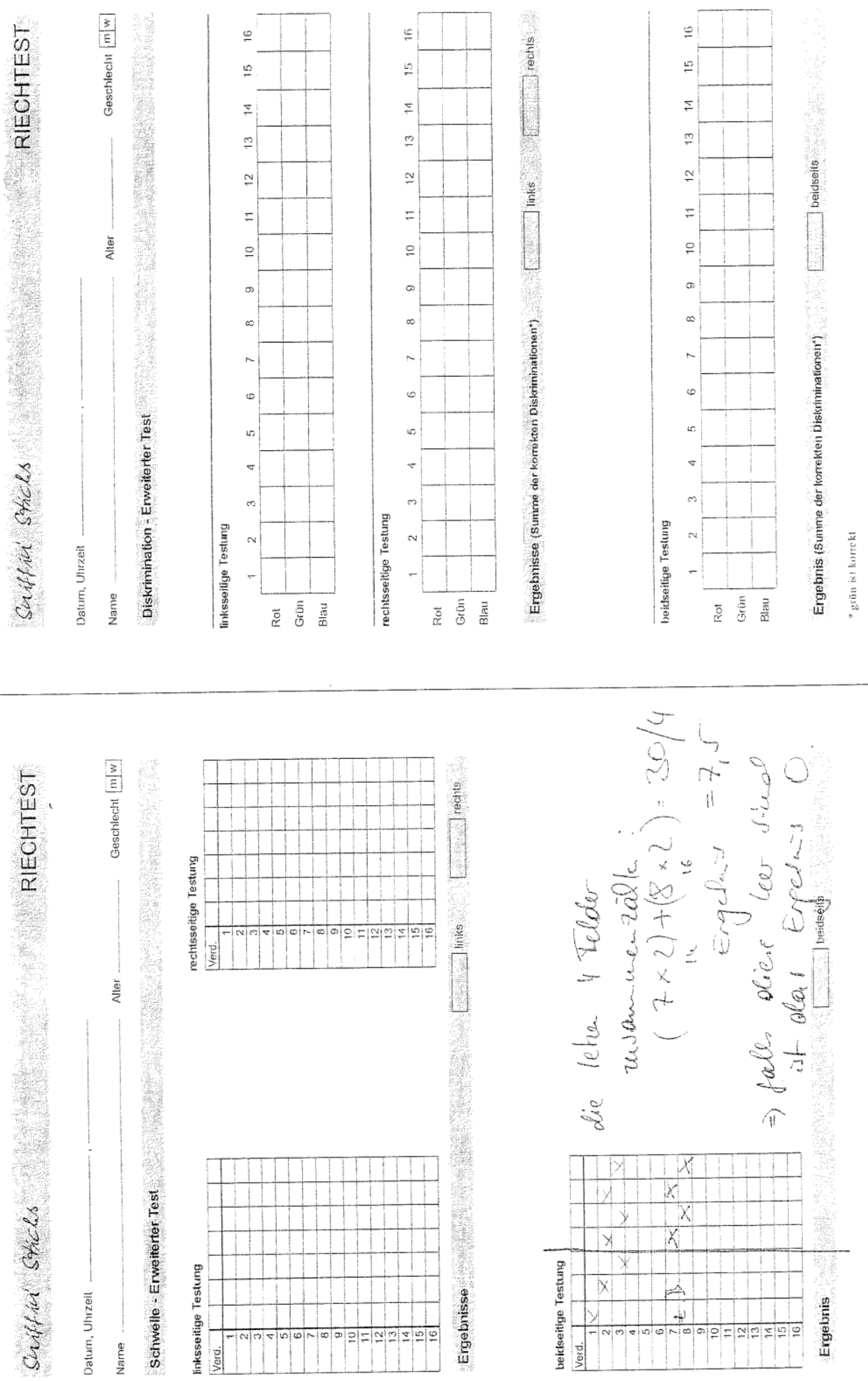

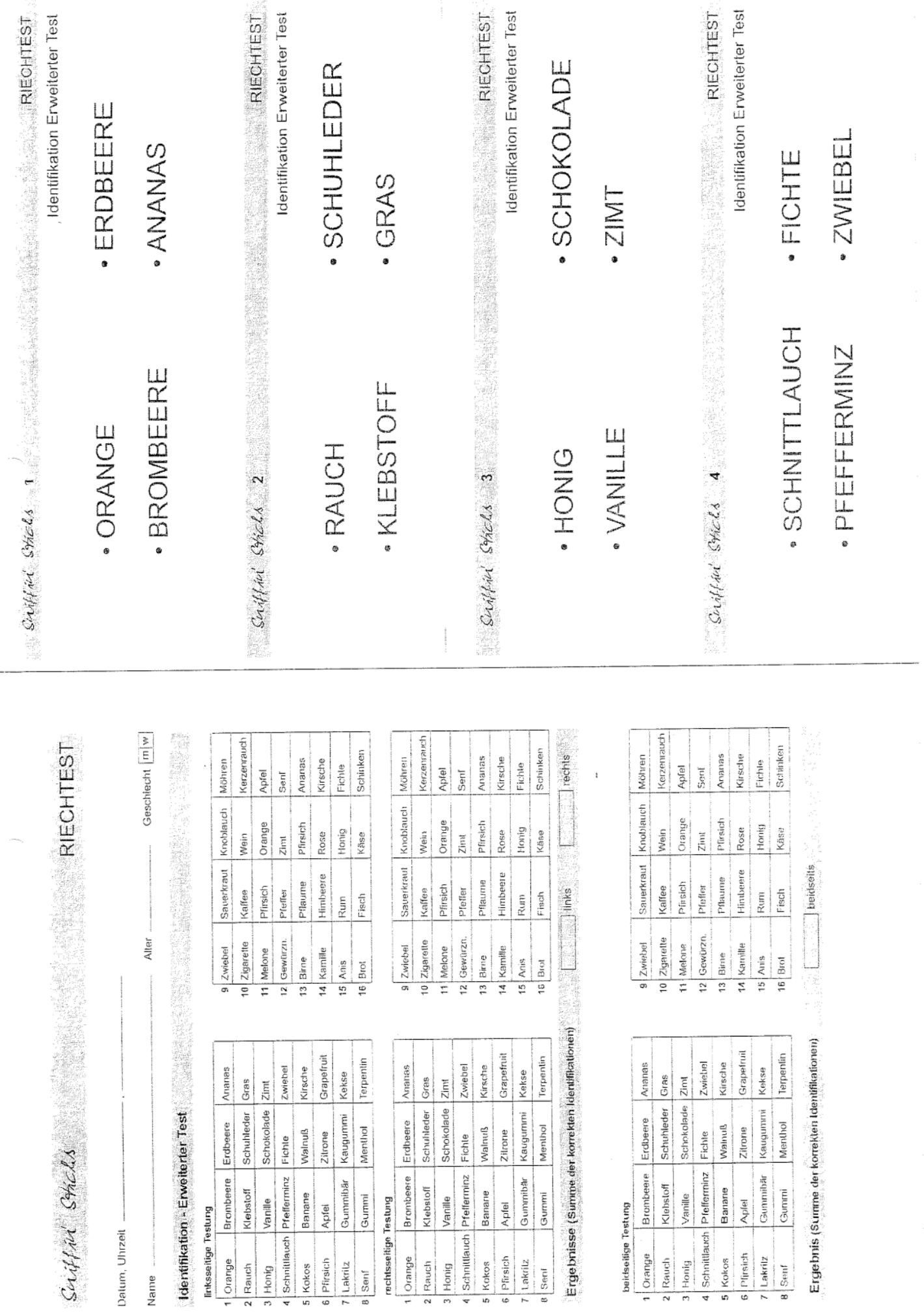

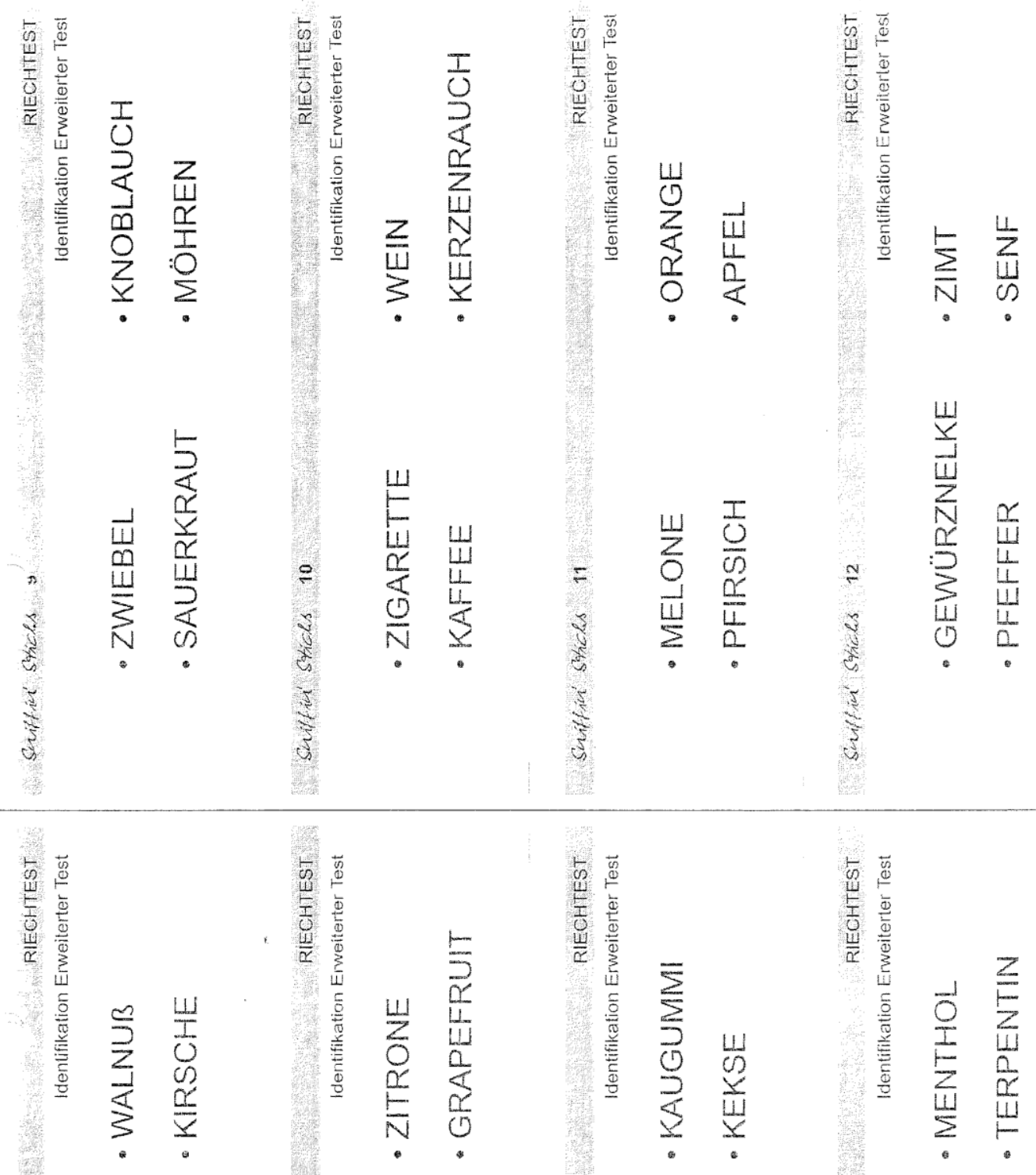

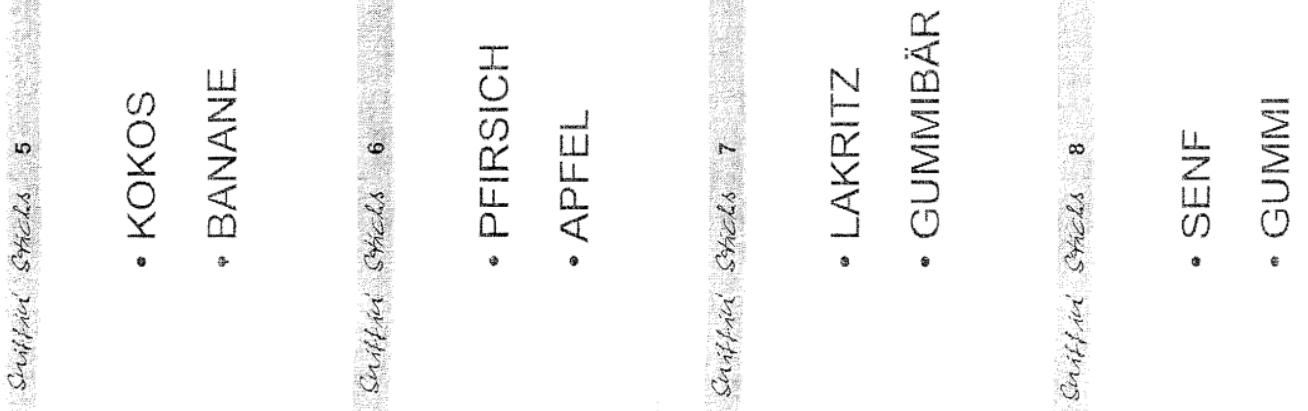




\section{MDS UPDRS}

\begin{tabular}{|c|c|c|}
\hline $1 . \mathrm{A}$ & Informationsquellen & $\begin{array}{l}\square \text { Patient } \\
\square \text { Betreuungsperson } \\
\square \text { Patient+Betreuungs- } \\
\text { person }\end{array}$ \\
\hline \multicolumn{3}{|c|}{ Teil I } \\
\hline 1.1. & Kognitive Beeinträchtigung & \\
\hline 1.2 & $\begin{array}{l}\text { Halluzinationen und } \\
\text { Psychose }\end{array}$ & \\
\hline 1.3 & Depressive Stimmung & \\
\hline 1.4 & Ängstliche Stimmung & \\
\hline 1.5 & Apathie & \\
\hline 1.6 & $\begin{array}{l}\text { Merkmale eines Dopamin- } \\
\text { Dysregulationssyndroms }\end{array}$ & \\
\hline $1.6 a$ & $\begin{array}{l}\text { Wer hat den Fragebogen } \\
\text { ausgefüllt }\end{array}$ & $\begin{array}{l}\square \text { Patient } \\
\square \text { Betreuungsperson } \\
\square \text { Patient+Betreuungs- } \\
\text { person }\end{array}$ \\
\hline 1.7 & Schlafstörungen & \\
\hline 1.8 & Tagesschläfrigkeit & \\
\hline 1.9 & $\begin{array}{l}\text { Schmerz und andere } \\
\text { Sinnesempfindungen }\end{array}$ & \\
\hline 1.10 & Blasenstörungen & \\
\hline 1.11 & Verstopfungsprobleme & \\
\hline 1.12 & $\begin{array}{l}\text { Schwindelgefühl im } \\
\text { Stehen }\end{array}$ & \\
\hline 1.13 & Mattigkeit & \\
\hline \multicolumn{3}{|c|}{ Teil II } \\
\hline 2.1 & Sprache & \\
\hline 2.2 & $\begin{array}{l}\text { Speichelfluss und } \\
\text { Ptyalismus }\end{array}$ & \\
\hline 2.3 & Kauen und Schlucken & \\
\hline 2.4 & Essen & \\
\hline 2.5 & Ankleiden & \\
\hline
\end{tabular}

\begin{tabular}{|c|c|c|}
\hline 2.6 & Körperpflege & \\
\hline 2.7 & Handschrift & \\
\hline 2.8 & $\begin{array}{l}\text { Hobbys und andere } \\
\text { Aktivitäten }\end{array}$ & \\
\hline 2.9 & Wenden im Bett & \\
\hline 2.10 & Tremor & \\
\hline 2.11 & $\begin{array}{l}\text { Aufstehen aus dem Bett, } \\
\text { Aussteigen aus einem Auto } \\
\text { oder Aufstehen aus einem } \\
\text { tiefen Sessel }\end{array}$ & \\
\hline 2.12 & Gehen und Gleichgewicht & \\
\hline 2.13 & Blockaden beim Gehen & \\
\hline \multicolumn{3}{|c|}{ Teil III } \\
\hline $3 a$ & $\begin{array}{l}\text { Erhält der Patient } \\
\text { Medikamente? }\end{array}$ & $\square$ Nein $\square$ Ja \\
\hline $3 b$ & $\begin{array}{l}\text { Klinischer Status des } \\
\text { Patienten }\end{array}$ & $\square$ Off $\square$ On \\
\hline $3 c$ & $\begin{array}{l}\text { Nimmt der Patient Levodopa } \\
\text { ein? }\end{array}$ & $\square$ Nein $\square \mathrm{Ja}$ \\
\hline $3 . \mathrm{C1}$ & $\begin{array}{l}\text { Falls ja, wie viele Minuten } \\
\text { sind seit der letzten } \\
\text { Medikamenten-dosis } \\
\text { vergangen? }\end{array}$ & $\overline{\text { Minuten }}$ \\
\hline 3.1 & Sprache & \\
\hline 3.2 & Gesichtsaudruck & \\
\hline \multirow[t]{5}{*}{$3.3 a$} & Rigor-Nacken & \\
\hline & Rigor- ROE & \\
\hline & Rigor - LOE & \\
\hline & Rigor - RUE & \\
\hline & Rigor - LUE & \\
\hline $3.4 a$ & Fingertippen- rechte Hand & \\
\hline $3.4 \mathrm{~b}$ & Fingertippen - linke Hand & \\
\hline $3.5 \mathrm{a}$ & $\begin{array}{l}\text { Handbewegungen - rechte } \\
\text { Hand }\end{array}$ & \\
\hline $3.5 b$ & $\begin{array}{l}\text { Handbewegungen - linke } \\
\text { Hand }\end{array}$ & \\
\hline
\end{tabular}

DeNoPa 


\section{MDS UPDRS (Fortsetzung)}

\begin{tabular}{|c|c|c|}
\hline $3.6 a$ & $\begin{array}{l}\text { Pronation-Supination - } \\
\text { rechte Hand }\end{array}$ & \\
\hline $3.6 b$ & $\begin{array}{l}\text { Pronation-Supination - } \\
\text { linke Hand }\end{array}$ & \\
\hline $3.7 a$ & $\begin{array}{l}\text { Vorfusstippen - rechter } \\
\text { Fuß }\end{array}$ & \\
\hline $3.7 \mathrm{~b}$ & Vorfußtippen - linker Fuß & \\
\hline $3.8 a$ & $\begin{array}{l}\text { Beweglichkeit der Beine - } \\
\text { rechtes Bein }\end{array}$ & \\
\hline $3.8 \mathrm{~b}$ & $\begin{array}{l}\text { Beweglichkeit der Beine - } \\
\text { linkes Bein }\end{array}$ & \\
\hline 3.9 & Aufstehen vom Stuhl & \\
\hline 3.10 & Gangbild & \\
\hline 3.11 & Blockade beim Gehen & \\
\hline 3.12 & Posturale Stabilität & \\
\hline 3.13 & Körperhaltung & \\
\hline 3.14 & $\begin{array}{l}\text { Globale Spontaneität der } \\
\text { Bewegung }\end{array}$ & \\
\hline $3.15 a$ & Haltetremor - rechte Hand & \\
\hline $3.15 b$ & Haltetremor - linke Hand & \\
\hline $3.16 a$ & $\begin{array}{l}\text { Bewegungstremor - } \\
\text { rechte Hand }\end{array}$ & \\
\hline $3.16 \mathrm{~b}$ & $\begin{array}{l}\text { Bewegungstremor - linke } \\
\text { Hand }\end{array}$ & \\
\hline $3.17 a$ & $\begin{array}{l}\text { Amplitude des } \\
\text { Ruhetremors-ROE }\end{array}$ & \\
\hline $3.17 \mathrm{~b}$ & $\begin{array}{l}\text { Amplitude des } \\
\text { Ruhetremors- LOE }\end{array}$ & \\
\hline $3.17 \mathrm{c}$ & $\begin{array}{l}\text { Amplitude des } \\
\text { Ruhetremors-RUE }\end{array}$ & \\
\hline $3.17 d$ & $\begin{array}{l}\text { Amplitude des } \\
\text { Ruhetremors- LUE }\end{array}$ & \\
\hline $3.17 \mathrm{e}$ & $\begin{array}{l}\text { Amplitude des } \\
\text { Ruhetremors- } \\
\text { Lippen/Kiefer }\end{array}$ & \\
\hline 3.18 & $\begin{array}{l}\text { Konstanz des } \\
\text { Ruhetremors }\end{array}$ & \\
\hline \multicolumn{3}{|c|}{ Einfluss der Dyskinesien auf Teil III } \\
\hline & Traten Dyskinesien auf? & $\square$ Nein $\square$ Ja \\
\hline & $\begin{array}{l}\text { Hatten diese Einfluss auf } \\
\text { die Bewertung? }\end{array}$ & $\square$ Nein $\square$ Ja \\
\hline
\end{tabular}

\begin{tabular}{|c|c|c|}
\hline \multicolumn{3}{|c|}{ Hoehn und Yahr Stadium } \\
\hline & $\begin{array}{l}\text { 0: Asymptomatisch. } \\
\text { 1: Nur einseitige Beteiligung. } \\
\text { 2: Beidseitige Beteiligung ohne } \\
\text { Gleichgewichtsstörung. } \\
\text { 3: Leichte bis mäßig ausgeprägte } \\
\text { beidseitige Beteiligung; gewisse } \\
\text { Haltungsinstabilität, jedoch körperlich } \\
\text { unabhängig; braucht Unterstützung zum } \\
\text { Ausgleich beim Zugtest. } \\
\text { 4: Starke Behinderung; kann aber noch } \\
\text { ohne Hilfe gehen oder stehen. } \\
\text { 5. Ohne fremde Hilfe auf den Rollstuhl } \\
\text { angewiesen oder bettlägerig. }\end{array}$ & \\
\hline \multicolumn{3}{|c|}{ Teil IV: Motorische Komplikationen } \\
\hline \multirow[t]{4}{*}{4.1} & Dauer der Dyskinesien & \\
\hline & $\begin{array}{l}\text { 1. Stunden der Wachzeit } \\
\text { gesamt: }\end{array}$ & \\
\hline & $\begin{array}{l}\text { 2. Gesamtzahl von Stunden mit } \\
\text { Dyskinesien }\end{array}$ & \\
\hline & 3. \%Dyskinesien $=\left((2 / 1)^{*} 100\right.$ & \\
\hline 4.2 & $\begin{array}{l}\text { Funktionelle Beeinträchtigung durch } \\
\text { Dyskinesien }\end{array}$ & \\
\hline 4.3 & Dauer der OFF-Phase & \\
\hline 4.4 & $\begin{array}{l}\text { Funktionelle Beeinträchtigung durch } \\
\text { Fluktuationen }\end{array}$ & \\
\hline 4.5 & $\begin{array}{l}\text { Komplexität der motorischen } \\
\text { Fluktuationen }\end{array}$ & \\
\hline
\end{tabular}

\section{DeNoPa}




\section{Danksagung}

Ich möchte mich herzlich bei Prof. Dr. med. Brit Mollenhauer bedanken, die die Anfertigung dieser Dissertation ermöglicht und mich durch ihre hervorragende Betreuung begleitet hat. 


\section{Curriculum vitae}

Am 27.04.1987 wurde ich als jüngste Tochter von insgesamt vier Kindern von Dr. med. Kunibert Hippel und Birgit Hippel, geborene Schöpker, in Berlin geboren.

Ich besuchte in Berlin Charlottenburg von 1994 bis 1999 die Katholische Schule HerzJesu, darauf folgend das Gymnasium Katholische Schule Liebfrauen, an dem ich im Jahre 2006 mein Abitur ablegte.

Vor Beginn meines Studiums begann ich eine Ausbildung zur Medizinischen Fachangestellten. Diese beendete ich, nachdem ich mein Studium der Humanmedizin an der Georg-August-Universität zu Göttingen im Sommersemester 2008 begann. Im Dezember 2015 erhielt ich meine Approbation. Seit dem 01 . August 2016 bin ich als Assistenzärztin in der Augenklinik Berlin Marzahn tätig. 